\title{
MOTION ANALYSIS FOR IN-HOME GAIT AND BALANCE ASSESSMENT USING INEXPENSIVE VIDEO SENSORS
}

A Dissertation presented to the Faculty of the Graduate School

University of Missouri

In Partial Fulfillment

Of the Requirements for the Degree

Doctor of Philosophy

by

\section{FANG WANG}

Dr. Marjorie Skubic, Dissertation Supervisor

Electrical and Computer Engineering Department

University of Missouri

JULY 2011 
The undersigned, appointed by the dean of the Graduate School, have examined the dissertation entitled

\section{MOTION ANALYSIS FOR IN-HOME GAIT AND BALANCE ASSESSMENT USING INEXPENSIVE VIDEO SENSORS}

Presented by Fang Wang,

A candidate for the degree of Doctor of Philosophy

And hereby certify that, in their opinion, it is worthy of acceptance.

Dr. Marjorie Skubic

Dr. James Keller

Dr. Marilyn Rantz

Dr. Tony X. Han 
This dissertation is dedicated to Hamzah, Omar and Mahmoud, and to all my family. 


\section{ACKNOWLEDGEMENTS}

I would like to express my sincere gratitude and appreciation to my advisor, Dr. Marjorie Skubic, for her most valuable guidance, caring, patience, and encouragement during the course of this research and my Ph.D study, and for her providing me with an excellent atmosphere for doing research. I am and will always be grateful that she took me into her research group when I was feeling lost and unsure about this complex environment at the beginning my Ph.D years.

I would also like to thank Dr. James Keller, Dr. Marilyn Rantz for guiding my research for the past several years and helping me to develop my background in eldercare technology and computer intelligence. I am thankful to Dr. Tony Han and the rest of the committee members for reviewing the manuscript and making guiding comments.

I would like to acknowledge the Eldertech research group members for their technical support and friendship, especially Wenqing Dai, Erik Stone, Jarod Giger, and Shang Wang.

I would like to acknowledge NSF for providing funding which made this study possible.

Finally, the acknowledge would not be complete without a heart-felt thanks to my entire family, whose unwavering love, faith and support have accompany me along the journey of this research. My deepest thanks go to my parents Zhimei and Xiaoci Wang, who have given me their fullest support and have helped so much with baby-sitting, my understanding and patient husband, Mahmoud, who has been a constant source of strength and inspiration to me, my precious little boys have made my life more joyful and meaningful than ever before. None of this would have been possible without them. 


\section{TABLE OF CONTENTS}

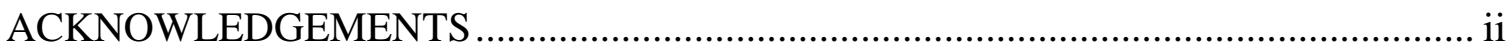

LIST OF ILLUSTRATION ………………………………………………………....

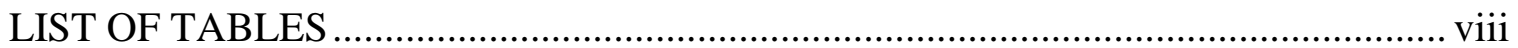

ABSTRACT

Chapter 1. Introduction.......................................................................................

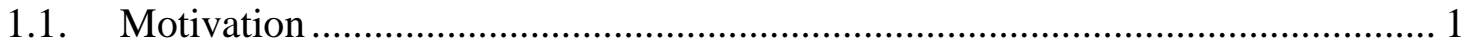

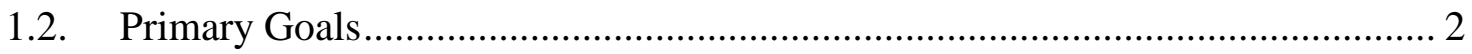

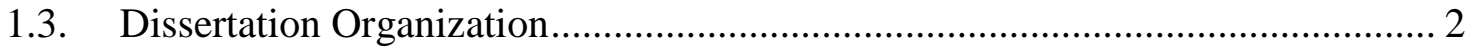

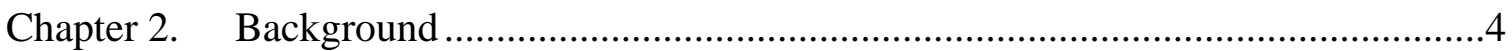

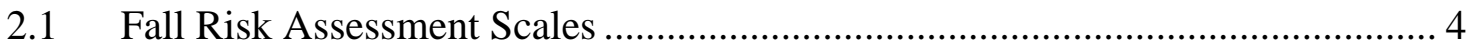

2.2 Gait and Balance Assessment Technology ....................................................... 7

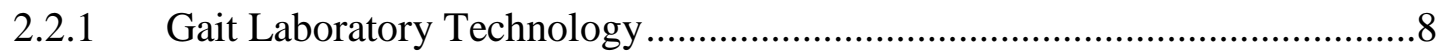

2.2.2 Mobility Assessment Technology Outside the Gait Laboratory...................10

a) Wearable Sensor Systems ........................................................................11

b) Passive and Unobtrusive Monitoring Systems ...........................................13

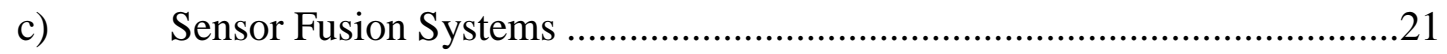

2.3 Three Dimensional Voxel Reconstruction from Silhouettes............................... 24

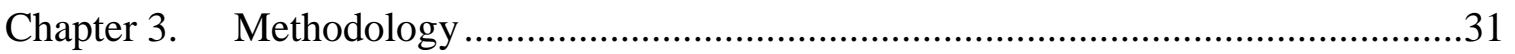

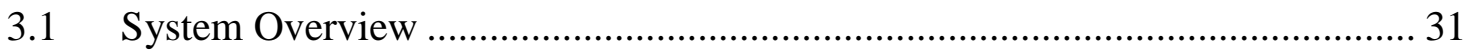

3.2 Two-Person Scenario Tracking …………………..................................... 32

3.3 Activity Segmentation ................................................................................ 36

3.4 Extraction of Gait Parameters ..................................................................... 42

3.4.1 Gait Parameters from Cameras ...................................................................42

3.4.2 Capturing Vicon Gait Parameters for Validation........................................50

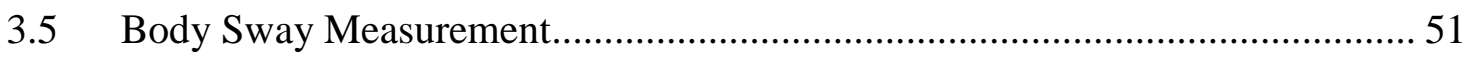

3.5.1 Body Sway during Standing ..................................................................51

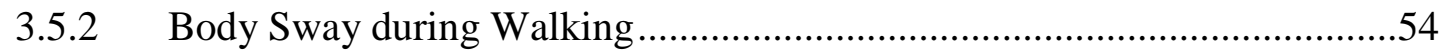

3.5.3 Vicon Marker-Based Motion Capture for Ground Truth...............................55

3.6 Turn Analysis and Turn Recognition ............................................................ 57 


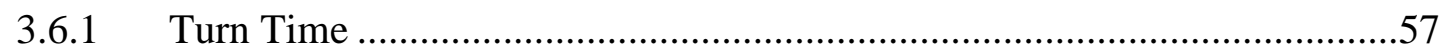

3.6.2 Number of Turn Steps.......................................................................59

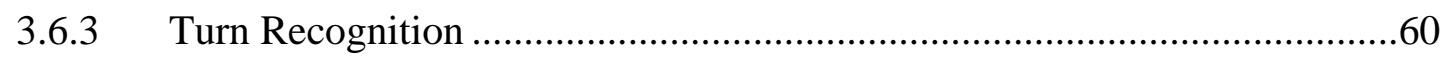

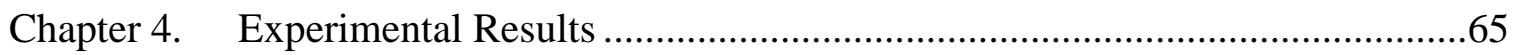

4.1 Validation Experiment for Gait Analysis ………………………………......... 65

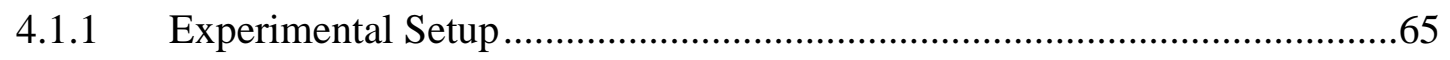

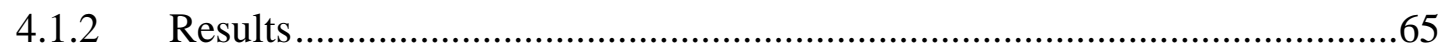

4.2 Validation Experiments for Body Sway........................................................ 71

4.2.1 Sway during Standing ............................................................................

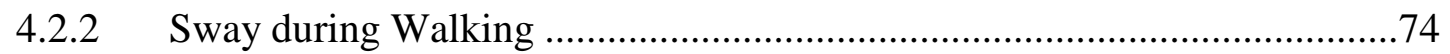

4.3 Validation Experiment for 180 Degree Turn Analysis ..................................... 76

4.4 Gait Analysis Testing with Elderly Participants $-1^{\text {st }}$ Round Testing................ 80

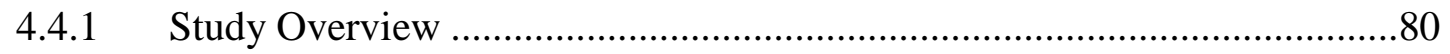

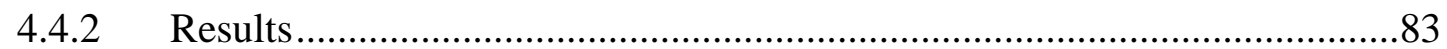

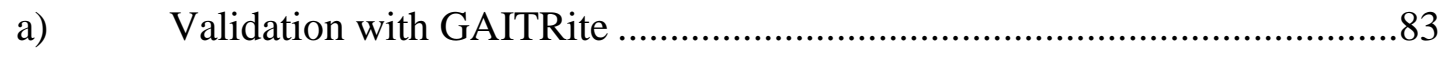

b) Gait Assessment with Realistic Scenarios ..................................................84

4.5 Gait Analysis Testing with Elderly Participants $-2^{\text {nd }}$ Round Testing ............... 89

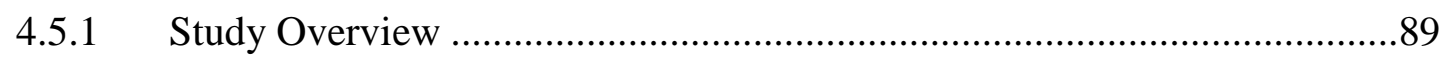

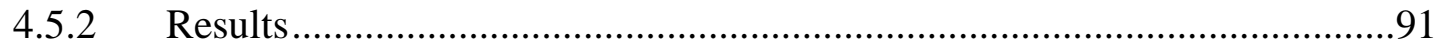

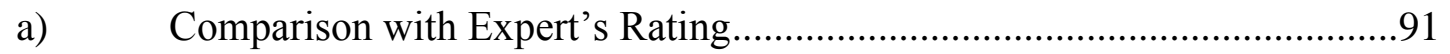

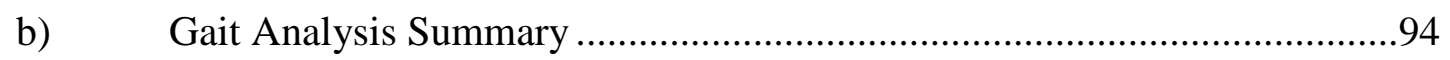

Chapter 5. Discussion ...........................................................................................100

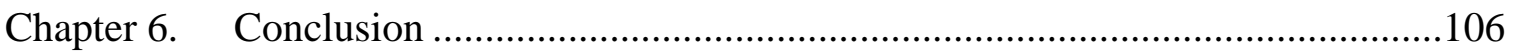

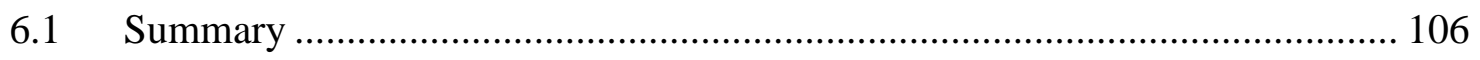

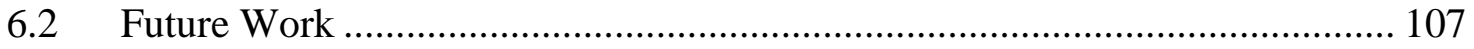

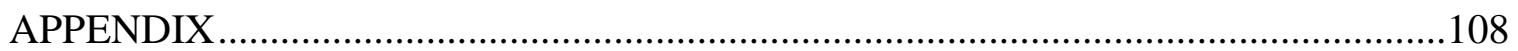

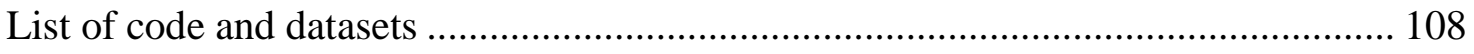

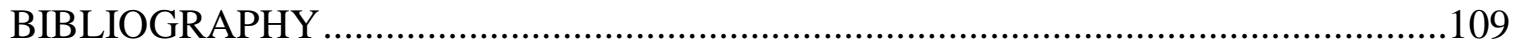

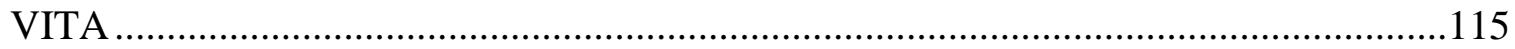




\section{LIST OF ILLUSTRATION}

Figure

Page

2.1 World and image coordinates systems.... .25

2.2 (a) Backproject each silhouette and intersect backprojected volumes (b) Color voxel black if on silhouette in every image.

2.3 (a) Two raw camera images monitoring the same scene. (b) Human silhouettes (c) The reconstructed three-dimensional voxel person

3.1 High level system overview of the proposed motion analysis system for gait and balance assessment...................................................... 31

3.2 Original images and tracking silhouettes illustration. In silhouette, red is for the resident, and green is for visitor .36

3.3 ID4 Test 2 velocity overview (a)velocity (b)cubic spline smoothing velocity used to segment walk.

3.4 Illustration of frame segmentation based foot area size and voxel person volume using ID5 run1 from 2nd round TigerPlace dataset as an example (a) Food area size (b) Voxel person volume (d) Frame 200, lower body occluded (e) Frame 779, lower body occluded (f) Frame 115, bad backprojection region (f) Frame 286, bad backprojection region. 41

3.5 (a) A projection of a valley frame, i.e., feet are together (b) A projection of a peak frame, i.e., feet are separated (c) Step length variation at different frames. .45

3.6 Voxel person body centroid projected in 2D space during a walk. Red circle: double support, Blue cross: right/left foot single support. Angles between red and blue arrows are extracted, as shown in b. (b) Angle values. Negative for right foot, positive for left foot. .47

3.7 Illustration of the cane for ID5Run1frame 780-781: ground projection of foot area before and after cane removal 
3.8 The projected 2D trajectory of the Vicon markers attached to a participant's feet, with footfall locations marked ( $\mathrm{x}$ for left foot and + for right foot) ............... 51

3.9 (a) Two raw camera images monitoring the same scene. (b) Human silhouettes (c) The reconstructed three-dimensional voxel person. The reference point and body

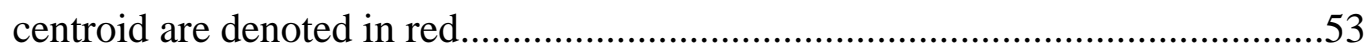

3.10 Sample sway amplitude curve during standing. Red crosses mark the maximum

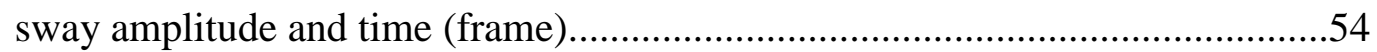

3.11 (a) Angle between 3-D vectors (b) Sample lateral sway angle during standing. Red crosses mark the maximum sway angle and time (frame)............................54

3.12 (a) Voxel person during walking (b) Body centroid trajectory during walking projected onto 2-D space. (c) Sway amplitude in med-lateral direction. Red crosses mark the maximum sway angle and time (frame)...................................56

3.13 (a) A person's walk and turn trajectory during the TUG test. (b) Velocity vector

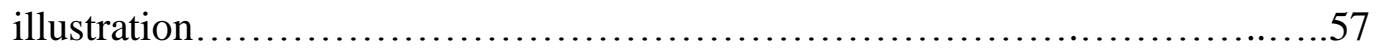

3.14 (a) Angles extracted from velocity vectors. (b) Image 21-26 from one camera view detected as a turn using the model.............................................................59

3.15 (a) Step length variation during the walk and turn. (b) Images $25 \& 29$ from one camera view which are detected as peak step lengths during a turn.....................60

3.16 ID4 Test 2 velocity overview (a) velocity vector angles (b) cubic spline smoothing velocity angels used to segment turn around . .61

3.17 ID4 Test 2 turn around (a) voxel person trajectory projected on ground plane with turn around frames marked (b) turn around images illustration: frame 1311, 1704 , and 1824.

4.1 (a)Velocity (b) Step Time (c) Step Length results for camera, GAITRite and

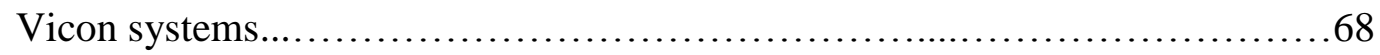

4.2 Sway comparison during standing: Webcam vs. Vicon (a) Sway amplitude (b) Sway angle vs. X-axis: anterior-posterior sway (c) Sway angle vs. Y-axis: lateral sway . .73

4.3 Sway comparison during walking for ID2 slow walk (a) Body centroid 2-D trajectory: Webcam vs. Vicon (b) Webcam: sway amplitude (c) Vicon: sway

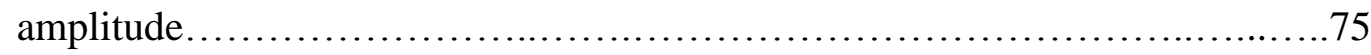

$4.4 \quad$ Turn time comparison......................................................... 77 
4.5 Turn step comparison. Number of test runs associated with each data points is listed beside the data point................................................................................79

4.6 Resident in scenario. (a) Two cameras monitoring the same scene. (b) Extracted human silhouettes. (c) Three dimensional voxel person reconstructed from the

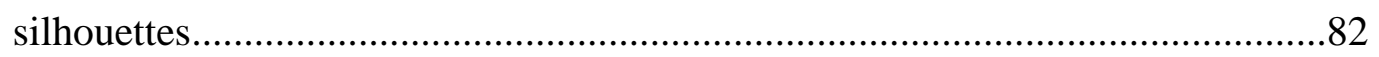

4.7 Webcam vs. GAITRite for TigerPlace residents..............................84

4.8 Resident in realistic scenario. (a) Sample voxel persons in sequence (b) Walking trajectory projected in 2-D space. Each point represents the centroid location in a

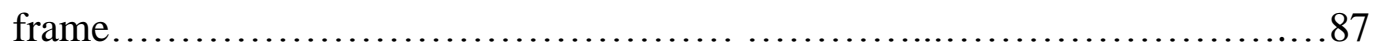

4.9 Gait parameters extracted by the algorithm are compared with the expert rating.(a) Velocity (b) Step time (c) Step length..............................94

4.10 Gait Analysis Results for 10 elderly participants. The gait parameter average values and their standard deviation (a) Velocity (b) Step Time (c) Step Length (d) Lateral sway amplitude 


\section{LIST OF TABLES}

$\begin{array}{ll}\text { Table Page } & \end{array}$

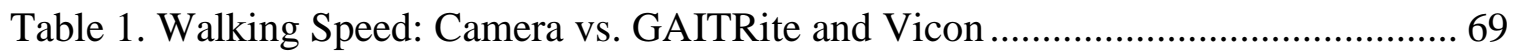

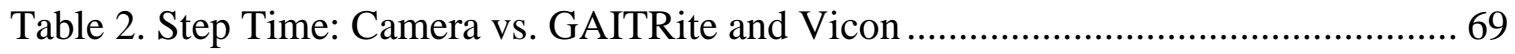

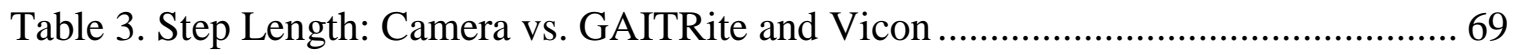

Table 4. Statistical Test (T-test) for camera, GAITRite and Vicon results .................... 70

Table 5. Right/Left Step Length Difference ...................................................... 70

Table 6. Sway Amplitude Comparison while Standing ........................................... 74

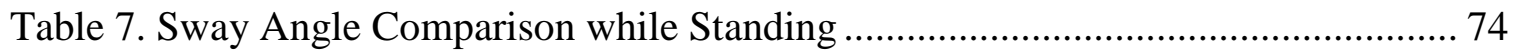

Table 8. Sway Amplitude Comparison while Walking ............................................ 76

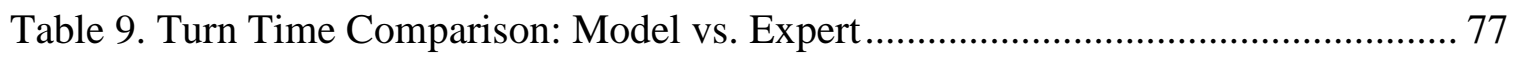

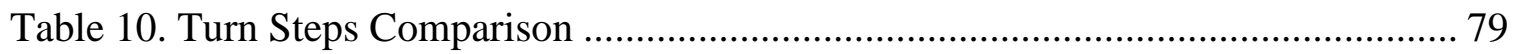

Table 11. Cameras (cam) Compared with GAITRite (GR) at TigerPlace with Resident

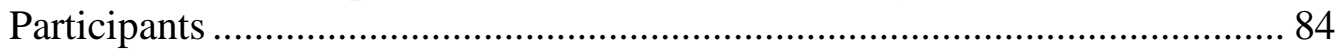

Table 12. Scenario Walks Compared with GAITRite Tests for Participants 1-5. Participants Walked Faster in the GAITRite Runs. .................................... 88

Table 13. Scenario Walks Compared with GAITRite Tests for the Participants 6-8...... 89

Table 14 Camera vs. Expert Comparison for 10 Feet Walk at TigerPlace...................... 94 


\title{
MOTION ANALYSIS FOR IN-HOME GAIT AND BALANCE ASSESSMENT USING INEXPENSIVE VIDEO SENSORS
}

\author{
Fang Wang \\ Dr. Marjorie Skubic, Dissertation Advisor
}

\begin{abstract}
Gait has been studied in recent years as one of the newest topics in computer vision as it offers potential for applications in various areas, including biometric identification, medical diagnostics and therapeutic tools. Clinical research has identified a clear link between human gait characteristics and different medical conditions. We are particularly interested in gait and balance analysis for fall risk assessment in elderly people. This dissertation proposes the use of multiple inexpensive web cameras for three-dimensional gait and balance analysis in a voxelized space. The main advantages of this approach include low cost, unobtrusive monitoring compared to wearable sensors, and threedimensional voxel reconstruction which provides a non-camera view-dependent capability. It is thus suitable for daily assessment and continuous monitoring of elderly people's non-controlled daily living environment.

The gait parameters studied include walking speed, step time and step length; balance was assessed through body sway. In addition, $180^{\circ}$ turnaround time and number of steps are also studied. These parameters were obtained from a three-dimensional voxel reconstruction, which is built from two calibrated camera views. These parameters are validated with a GAITRite Electronic mat, a Vicon motion capture system in the lab, and a physical therapy expert rating. Excellent agreements were found for walking speed, step
\end{abstract}


time, and step length between the webcam system and the GAITRite, with no statistically significant difference. There is no significant difference in walking speed and step length comparing the webcam system with the Vicon. Significant difference was observed for step time between the webcam system and the Vicon, as well as between the GAITRite and the Vicon.. There is no significant difference in walking speed between the system and the expert, but highly significant difference in step time and step length, caused by the expert's strategy of rounding up the number of steps when there is an incomplete gait cycle. Overall, the walking speed was found to be the most robust and reliable gait parameter captured through all the tests. The body sway and turnaround parameters were also found to have a reasonably good agreement given the limitation of frame rate and voxel resolution.

The system was further tested and validated with assistance of elderly residents from TigerPlace, an independent living apartment complex for seniors in Columbia, Missouri, in scripted scenarios representing everyday activities. The residents were first tested on a GAITRite mat with cameras recording images. The extracted gait parameters from the camera system were compared with the GAITRite; and excellent agreement was achieved. The residents then participated in the scenarios, with only the cameras recording. Parts of the scenario video of 10 participants were evaluated and rated by a physical expert in the research team. The algorithm results matches well with the expert rating. Based on these good validation results obtained in a realistic non-lab setting, further analysis is therefore reliable. Results on 18 elderly participants were analyzed. The analysis showed that the residents displayed different gait patterns when the realistic scenarios were compared to the GAITRite runs. Furthermore, the complete set gait parameters provided a clear 
picture of a person's gait status and were used to screen out people at risk of falling. This research shows how long-term gait monitoring can provide trend information on a person's gait characteristics and proves the importance of continuous gait assessment in a daily living environment.

Research results indicate that the development of this technology can provide capability of monitoring gait and balance in daily living environment for elderly people, which in turn can be used as part of a continuous balance, stability and fall risk assessment tool. 


\section{Chapter 1. Introduction}

\subsection{Motivation}

A rapidly expanding aging population impacts the health care services around the globe. Research focused on older adults continues to escalate, especially regarding how to promote successful aging, enhance the overall quality of life and provide adequate medical care while keeping health care cost under control. One approach is to employ technology such as wireless networks combined with novel sensing systems to gather and interpret data in non-healthcare settings such as homes. Many such systems have been proposed to assist the elderly. One specific measure of particular interest is the unobtrusive assessment of the mobility and fall risk for elderly health monitoring.

Falls are one of the most common health concerns facing elderly persons today. About one-third of community-dwelling persons over the age of 65 and nearly one-half of institutionalized persons will fall each year. Not only do falls result in injury, falls may also lead to increased fear of falling and restricted social and physical activity which drastically reduces ones quality of life. A rapidly aging population, expensive medical care and overwhelmed healthcare systems drive the need to develop a means of identifying the at-risk population, delivering appropriate interventions and reducing the fall rates and risks within the elderly population.

One of the strongest risk factors in determining fall risk is that of impaired balance, mobility, and gait [1]. Prospective studies have demonstrated that tests of standing, leaning, reaching, stepping, and walking can distinguish adults who fall from those who 
do not fall, with more challenging balance tasks having stronger association with the risk of falling $[1,2]$.

Many gait and balance assessment systems are expensive, and only suitable for lab or clinic settings. There have been no low-cost passive sensor systems available to provide a reliable and quantifiable method of monitoring the gait parameters in daily activities. This research developed a two-camera system to address gait assessment as described above using inexpensive web cameras. Using 3D voxel data eliminated the limitation of a controlled walking path making it suitable for daily assessment in the home environment.

\subsection{Primary Goals}

The principle objectives of this research include:

- Feature extraction using the three-dimensional human object reconstruction in voxel space.

- Human tracking in single and two-person scenarios.

- Motion analysis

○ Gait

○ Body Sway

○ 180 Degree Turn

\subsection{Dissertation Organization}

Chapter 2: Background

Previous research in fall risk assessment has produced a plethora of fall risk assessment scales and technologies inside and outside the lab. This chapter will include a 
review of the most common fall risk assessment scales, gait laboratory technologies, and technologies outside the lab including wearable and non-wearable sensors as well as sensor fusion technology. Silhouettes and 3D voxel person reconstruction techniques used are also reviewed.

\section{Chapter 3: Methodology}

This chapter describes the current status of the project, including two-person tracking, gait and balance parameters extraction methodology.

\section{Chapter 4: Experimental Results}

This chapter describes the current experiments setup and results.

Chapter 5: Discussion

Chapter 6: Conclusion 


\section{Chapter 2. Background}

This chapter includes a review of the current gait and balance assessment tools including fall risk assessment scales commonly used by physical therapists, technology used in laboratories, and technology used outside of laboratories. In addition, this project's silhouettes and 3D voxel person reconstruction techniques are reviewed.

\subsection{Fall Risk Assessment Scales}

The purpose of this section is to summarize existing fall risk assessment scales to enable more informed choices regarding their use with the camera systems set up for this research.

Because of the extreme cost both to the patient and to society, much work has been done to develop preventive programs throughout the continuum of care. Although there are many interventions proposed for fall prevention depending on the patient population, the initial step for virtually all of these programs is the fall risk assessment, which is performed to identify persons at highest risk upon whom to target specific interventions. Fall risk assessment, however, is not standardized within or across settings. Traditionally, three types of assessments relevant to falls and mobility have been done, usually on the basis of setting or specific discipline factors. These include (i) comprehensive medical assessments performed by geriatricians or nurse practitioners

in the outpatient or nursing home setting, (ii) nursing fall risk assessments completed in hospital and nursing home settings, and (iii) functional mobility assessments completed by physical therapists or physicians in an outpatient setting. The functional mobility assessment is a primary research priority in this dissertation and is discussed below. 
Fall risk assessment of community-dwelling, elderly individuals based on functional assessment instruments has become common. These instruments focus on functional limitations in gait and balance, e.g., Tinetti Performance Oriented Mobility Assessment [3], Berg Balance Test [4], Functional Reach [5], or Dynamic Gait Index [6]. Few instruments have specifically tested the ability to predict falls, but all provide standardized measures of disability and functional limitations. These functional assessment tools are most widely performed by physical therapists for outpatient, community-dwelling patients. Some of these tools are quite detailed and can be burdensome to the patient, requiring the patient to walk, get up from a chair, and/or perform other functional activities that can take up to 20 minutes to complete. In clinical settings, poor scores typically trigger functional interventions, e.g., muscle strengthening gait/balance training, or aerobic exercise. The following assessment scales have been identified by the physical therapist experts in the University of Missouri-Columbia Center for Eldercare and Rehabilitation Technology (CERT) directed by this dissertation's advisor Dr. Marjorie Skubic and Dr. Marilyn Rantz as the fall risk assessment protocol in this research project.

\section{Forward Reach:}

The Forward Reach assessment tool has the patient stand next to the tape measure at the zero mark on the wall. The feet should be shoulder width apart and the right arm must be held forward at shoulder level. Patient must mark the tape with middle finger after reaching forward as far as possible without losing balance.

\section{Berg Balance Scale (BBS):}


The Berg Balance Scale was developed to measure older people's balance impairment by assessing their performance of functional tasks. It is a valid instrument used for evaluating the effectiveness of interventions and for quantitative descriptions of function in clinical practice and research. The BBS has been evaluated in several reliability studies. A recent BBS study completed in Finland indicates that a change of eight BBS points is required to reveal a genuine change in function between two assessments of older people who are dependent in Activities of Daily Living (ADL) and living in residential care facilities.

The testing items include:

- Sitting to standing

- Standing unsupported

- Sitting unsupported

- Standing to sitting

- Transfers

- Standing with eyes closed

- Standing with feet together

- Reaching forward with outstretched arm

- Retrieving object from floor

- Turning to look behind

- Turning 360 degrees

- Placing alternate foot on stool

- Standing with one foot in front

- Standing on one foot

\section{Dynamic Gait Index(DGI):}

The purpose of this assessment tool is to evaluate and document a patient's ability to modify gait in response to changing task demands. Target subjects are ambulatory patients with balance impairments, i.e., older community-dwelling adults with vestibular dysfunction. The DGI effectively predicts the likelihood for falls in this target group.

The testing items include:

- Normal walking speed

- Gait on level surface

- Change in gait speed 
- Gait with horizontal head turn

- Gait with vertical head turn

\section{Timed Get Up \& Go (TUG):}

The timed get up and go (TUG) assessment test is a measurement of mobility. It includes a number of tasks such as standing from a seating position, walking, turning, stopping, and sitting down which are all important tasks needed for a person to be independently mobile.

This test requires the patient to stand up from a standard chair and walk a distance of approximately 10 feet (measured as 3 meters), turn around, walk back to the chair and finally sit down again. The individual uses his/her usual footwear and can use any assistive walking device they normally use, such as a cane. The person is seated with his/her back to the chair, their arms resting on the arm rests, and any walking aid they regularly use readily available. . Timing, using either a wristwatch with a second hand or a stop watch, begins when the patient starts to rise from the chair and ends when he/she is once again seated in the chair.

The normal time required to finish the test is between $7-10$ seconds. Individuals who cannot complete the task in that time frame probably have some mobility problems, especially if they take more than 20 seconds. This information should be documented as a baseline and repeated if any change in mobility occurs or at least yearly.

\subsection{Gait and Balance Assessment Technology}

One single assessment technique cannot be claimed and used as a true indicator of the overall integrity of the gait and balance control system. Some fall risk assessment scales are easy to perform and suitable for daily clinical use, but not always accurate enough. 
Studies have shown that these clinical tests can be subjective and sometimes inconsistent, especially when the testers are inexperienced [7,8]. In addition, they are usually not administrated often enough to identify the problem while they are small before they become big problems and offer timely interventions. Therefore, new technologies are necessary to give more detailed information about gait and balance assessment.

Most of the current gait and balance assessment systems can be categorized into wearable devices, walk-on devices and vision-based devices and techniques. Based on application settings, the following review categorizes them into two categories: 1) in lab/clinic settings and 2) in unobtrusive home and daily use settings.

\subsubsection{Gait Laboratory Technology}

Gait analysis commonly involves measurement of body movement in space (kinematics) and the forces involved in producing these movements (kinetics).A gait laboratory [9] typically uses a combination of three technologies to evaluate the biomechanics of gait: 1) A motion capture system tracks the patient's movements digitally; 2) force plates are used to measure the ground reaction force (GRF); and 3) an electromyography (EMG) system is used to record the activity of the muscles in gait.

A typical modern gait lab has several cameras (video and/or infra-red) placed around a walkway or treadmill which are linked to a computer. The patient has single markers applied to anatomical landmarks, such as palpable bony landmarks (e.g., the iliac spine of the pelvis, the malleoli of the ankle, and the condyles of the knee), or clusters of markers applied to the middle of body segments. The patient walks down the walkway or the treadmill and the computer calculates the trajectory of each marker in three dimensions. 
A model is applied to compute the underlying motion of the bones. This gives a full breakdown of the motion at each joint.

In addition, to calculate movement kinetics, most laboratories have floor-mounted load transducers, also known as force platforms, which measure the ground reaction forces including magnitude, direction, and location (called center of pressure). Adding these data to the known dynamics of each body segment, enables the solution of equations based on Newton's laws of motion and enables the computer to calculate the net forces and the net moments of force around each joint at every stage of the gait cycle. The computational method for this is known as inverse dynamics.

This use of kinetics, however, does not result in information for individual muscles but instead covers muscle groups, such as the extensor or flexors of the limb. To detect individual muscle activity and contribution to movement, it is necessary to investigate the electrical activity of muscles. Many labs also use surface electrodes attached to the skin to detect the electrical activity or EMG of, for example, of leg muscles. In this way it is possible to investigate the activation times of muscles and, to some degree, the magnitude of their activation-thereby assessing their contribution to gait. Deviations from normal kinematic, kinetic, or EMG patterns are used to diagnose specific conditions, predict the outcome of treatments, or determine the effectiveness of training programs.

Motion in combination with force-plates provides very accurate descriptions and models of gait. However, these expensive systems must be installed in appropriate rooms and can only be operated by specially trained personnel. Motion capture systems can only record movements performed in a small area of the room. Logging long walks is therefore only possible if the patient is walking on a treadmill, which may change the 
patient's normal gait. Other measuring systems used in the lab setting, more mobile but less informative, are pressure sensitive mats. Pressure mats provide static spatialtemporal data, analogous to footprints over time. They do not, however, provide dynamic information about how the patient's foot is moving in space. One commercially available solution to this problem is the GAITRite system. It was used in this research to provide ground truth. The GAITRite system automates measuring temporal (timing) and spatial (distance) gait parameters via an electronic walkway connected to the serial port of a personal computer. As the patient ambulates across the walkway, the system captures the geometry and the relative arrangement of each footfall as a function of time. The application software controls the functionality of the walkway, processes the raw data into footfall patterns, and computes the temporal and spatial parameters.

\subsubsection{Mobility Assessment Technology Outside the Gait Laboratory}

Gait analysis is already widely used for clinical assessment, but it is often subjective or constrained to a laboratory environment. As discussed earlier, gait analysis laboratories are large, complex and expensive facilities. The number of clinical gait analysis laboratories has increased, but the availability is still limited. The cost of full gait evaluation is not suitable for routine gait and balance assessment.

Another drawback of current approaches to clinical gait assessment is that tasks performed in the lab or clinic do not always simulate normal daily activities. And the gait laboratory walkway is not representative of the complex environment within which an elderly person must function. Consequently, such measurements may fail to reflect the subject's normal walking pattern. There is, therefore, a clear need for an inexpensive, unobtrusive and easy-to-use system, which allows continuous and quantitative analysis of 
gait patterns outside the lab. This research presents such technology and shows how it can be used as a diagnostic or screening tool to assess function and determine whether an intervention is needed or to determine whether an ongoing intervention is effective.

\section{a) Wearable Sensor Systems}

Pertinent background information on the current technology used to address fall risk patients i.e., wearable sensor systems, is defined in this section, although these alternative technologies are not the research priorities for this project.

\section{Accelerometers}

Researchers have traditionally deployed several techniques for continuous mobility assessment with various degrees of obtrusiveness. Among the least obtrusive of these techniques are those based on accelerometers attached to limbs.

Accelerometers are suitable sensors for wearable systems since current enhancements in micro-electro-mechanical systems (MEMS) technology have made possible the manufacture of miniaturized, low-power, low-cost accelerometers, which are useful for logging human motion data for long periods of time in uncontrolled environments. Accelerometers have been employed for many different purposes: estimating metabolic energy expenditure; monitoring activity; assessing standing balance; detecting falls, postural orientation and classifying activities [10]. Gait analysis studies from accelerometer sensor systems may be divided into three main categories:

1) The reconstruction of movements in space $[11,12]$. These studies make use of two or more sensors on the same limb in order to reconstruct its trajectory in space.

2) The detection of gait phases and evaluation of temporal parameters, e.g. $[13,14,15]$. These studies focus on detecting events such as heel-strike or stance and use this 
information to calculate stride times, variability, temporal symmetry, etc. They do not, however, provide any information about the way in which the person is walking, i.e. if the person's feet are moving according to different trajectories in space.

3) The classification of walking patterns, e.g. $[16,17]$.These studies aim at determining if the subject is walking up or down stairs, running etc. They do not, on the other hand, look into single steps and the phases of gait.

\section{GaitShoe}

Bamber, Benbasat, Scarborough, Krebs \& Paradiso [18] developed a shoe-with an integrated wireless wearable system to provide quantitative gait analysis outside the confines of the traditional motion laboratory. The sensor suite includes three orthogonal accelerometers, three orthogonal gyroscopes, four force sensors, two bidirectional bend sensors, two dynamic pressure sensors, as well as electric field height sensors. The "GaitShoe" was built to be worn in any shoe, without interfering with gait and was designed to collect data unobtrusively in any environment and over long time periods. The calibrated sensor outputs were analyzed and validated with results obtained simultaneously from the Massachusetts General Hospital Biomotion Laboratory. The GaitShoe was highly capable of detecting heel-strike and toe-off problems as well as estimating foot orientation and position.

\section{E-Textiles}

Electronic textiles (e-textiles) [19] are fabrics that enable computing, digital components, and electronics to be embedded in them. They offer the promise of home health care devices that integrate seamlessly into the wearer's everyday lifestyle while providing a higher level of functionality than current devices. This dissertation proposes 
an e-textile solution along with a design approach for realizing a solution that is inexpensive and usable across the elderly population. This research's preliminary results demonstrate the promise of the proposed system.

These wearable sensor systems typically consist of some wearable combination of sensors, such as gyroscopes and/or accelerometers and have demonstrated accuracy and precision in the field. However, these systems suffer from several limitations such as short battery life, the need to download the data or introduce additional hardware and complexity for wireless data collection, and the inconvenience of both a wearable device and having to remember to wear a device. For these reasons, wearable devices are currently considered inadequate for long-term, in-home, unobtrusive monitoring.

\section{b) Passive and Unobtrusive Monitoring Systems}

This section covers approaches and issues using vision and non-vision based sensors for the unobtrusive home monitoring of elders to determine location, speed of walking and daily activities which can detect early changes in cognitive function.

\section{i) Non-vision based gait analysis systems:}

Recently, researchers have started investigating low-cost passive sensor systems for gait assessment, such inexpensive passive infrared (PIR) sensors and a wireless network for continuous and unobtrusive assessment of mobility and walking speed in the home $[20,21,22]$.

Pavel, Hayes, Adami, Jimison, \& Kaye [20] of the University of Oregon's Biomedical Engineering Division in Portland examined a mobility assessment tool based on inexpensive PIR detectors. These detectors were designed to detect certain dynamic patterns of infrared energy within their field of view. Because PIR detectors monitor 
human activity, they must be unobtrusive, have low cost, low power consumption, and negligible maintenance. The low power consumption is achieved by having the detectors respond to onset of motion, followed by infrequent sampling. Each discrete response of each detector is transmitted via low power radio frequency to the base station. Despite their features, these detectors have serious shortcomings, the most serious of which is that their output is related only indirectly to the clinically relevant aspects of mobility such as the speed of walking. To overcome this serious shortcoming, a combination of approaches have been used which include modifying the sensors' field of view, designing a careful sensor placement and developing statistical pattern recognition algorithms. In addition, corresponding models have been used for estimating speed of walking. Due to the discrete characteristics of the observable sensor data, the framework chosen to represent the elder's movement was based on hidden semi-Markov model. Despite promising early results, these methods will require considerable future research, because there are numerous issues that have not been addressed by the described system most notably tracking individuals in multi-occupant dwellings. This dissertation's research filled a significant research gap by utilizing a multi-occupant elder-care complex for innovative development of new gait and balance assessment tools.

In addition, Hayes, Hagler, Austin, Kaye \& Pavel [21] and Hagler, Austin, Hayes, Kaye, \& Pavel [22], all part of the Oregon Health and Science University Biomedical Engineering research [20] proposed a system estimating walking speed from noisy time and location data collected by a "sensor line" of restricted view passive infrared (PIR) motion detectors. Depending on the architectural constraints, Hagler et al. [22] was able to measure the speed of walking by strategically placing several motion detectors along 
the walls of a hallway or a corridor. This placement of detectors enabled the measurement of walking speed in a relatively direct manner compared to Pavel et al. [20]. The Oregon system for continuous in home assessment of walking speed based on PIR sensors and a wireless network for data collection proved to be both accurate and precise when compared to the standard of the GAITRite walkway system. This method allows the convenient in-home collection of a large number of walking events, otherwise gathered infrequently in a clinical setting. However, such systems can provide only walking speed as the single gait parameter, and walk path is restricted along a straight sensor line.

A passive unobtrusive gait-monitoring device based on an ultra high sensitive opticfiber sensor that is capable of detecting vibrations generated by a person walking tens of feet away on both carpeted and uncarpeted wooden and concrete floors is presented by Alwan, Palal, Kell \& Felder [23]. Preliminary analog signal processing and some digital signal analysis has shown that the signature walking signals picked up from the floor in field testing allow for the distinction between normal, limping and shuffling gait modes. The device can accurately and reliably measure the number of steps taken by the person during normal walk, thus pace can be calculated. The device can also automatically detect falls. This gait monitor can be fixed in a corridor or a walkway within the person's home for long term monitoring of changes in gait mode and drifts in pace that may be precursors of a potential fall.

Low, Tani, Chanda, \& Want [24], proposed a home-based foot mat system. Each section of the foot mat consists of 16 Flexiforce sensors. The Flexiforce sensor acts as a force sensing resistor in an electrical circuit. With this daily monitoring system, the 
changes in gait characteristics, which are leading to proneness of falls and which are hardly distinguishable to the naked eye can be noticed. The reliability of the homesystem was also verified with a comparison to GAITRite. Finally, modeling experiments show the effectiveness of the monitoring system. In conclusion, a home-system having a reasonable price can be produced and is able to provide daily non-intrusive monitoring.

\section{ii) Vision-based gait analysis systems:}

Video sensors are a rich source of information that can be used for gait analysis; and vision-based gait analysis has been one of the most active research topics in the computer vision community. Applications for vision-based gait analysis seem to fall into the following categories: gait recognition as a biometric, gait classification, and gait and balance assessment as a tool in elder care, rehabilitation and sports activity. Currently, more studies using cameras are for gait classification and recognition purposes. A good background review on vision-based human motion analysis techniques is provided by Aggarwal and Cai [25] and Moeslund et. al[26].

In dealing with the problem of gait recognition for personal identification, human gait is appealing for two reasons: In contrast to fingerprint or retina scans, gait can be analyzed from a distance but is yet as significant as the former. The fact that even though no two body movements are ever the same, gait is a unique personal characteristic was first noted by Bernstein in the 1920s. Medical studies from the 1960s systematically corroborated this observation and psychological experiments conducted in the 1970s revealed that humans effortlessly recognize people by the way they walk. The spectrum of methods that have been proposed in vision based gait analysis is vast. Of the current approaches, most are based on silhouettes analysis and can be broadly split into model- 
based and model-free approaches. The model-based approaches use information about the gait determined either by known structure or by modeling. And the model-free approaches can be split into those based on a moving shape or those which use integrated shape and motion within the description. One advantage of model-based method is immunity to the effects of clothing and to slight change in viewpoint but usually at the cost of greater computational complexity. Detailed review of these biometrics can be found in Nixon, Tan and Chellappa's valuable historical resource, Human Identification Based on Gait [27].

Gait classification aims essentially to distinguish between different types of gait behaviors such as walking, running and jumping, e.g. Meyer, Psl and Niemann [28] extracted the feature vectors from optical flow trajectories of the tracked body parts to perform gait classification, and Bissacco, Chioso, Ma and Soatto [29] recognized different types of gaits in the space of dynamics.

Bauckage et al. in [30] and L. Wang [31] present gait analysis from another angle to examine its possible use as a means to deduce the physical conditions of people by detecting abnormal gait. Their research covers a totally different problem, namely determining different styles of the same walking action of a specific subject and further deciding whether or not the subject deviated from normal walking pattern. The proposed method in [31] starts with the extraction of human silhouettes from input videos and the computation of frame-to-frame optical flows, then motion metrics based on histogram representations of silhouette-masked flows, and finally gait analysis with eigenspace transformation. Differing from current gait classification and recognition studies, the proposed method deals with another interesting problem, namely not only determining 
different styles of the same walking action but detecting whether or not a walking style deviates from its usual walking pattern, which is expected as a feasible means to deduce physical conditions of people.

Understanding the detection of unusual movement patterns as a two class problem leads to the idea of using support vector machines for classification [30]. The authors present a homeomorphism between 2D lattices and binary shapes that provides a robust vector space embedding of body silhouettes. Experimental results will underline that feature vectors obtained from this scheme are well suited to detect abnormal gait. Wavering, faltering, and falling can be detected reliably across individuals without tracking or recognizing limbs or body parts.

Extensive research has been conducted to investigate the use of computer vision techniques and low cost video based systems for monitoring and assessing daily activities of the occupants. For example, Nait-Charif and McKenna [32] presented a simple vision system in a supportive home environment for activity recognition and fall detection. Gao, Wactlar \& Hauptmann [33] proposed a technique of fusing motion segmentation with tracking for eating behavior analysis of patients in a nursing home. From a rehabilitation and fall risk assessment standpoint, gait analysis can be used to identify musculoskeletal deficiencies and to develop programs for using the information to implement better elder care.

The UbiSense project [34] aims to develop an unobtrusive health monitoring system for the elderly by using embedded smart vision techniques. In addition to monitoring normal daily activities and detecting potential adverse events such as falls, the UbiSense system aims to capture signs of deterioration of the patients by analyzing subtle changes 
in posture and gait. For example, in the case of Parkinson's disease there can be the shuffling signs and other related behaviors such as a reduced arm swing on one side when a person is walking caused by rigid muscles. Each UbiSense node consists of an embedded smart camera VCSBC50 (a DSP camera with the ADSP 2185), a wireless link provided by an integrated BSN node, and a battery.

Markerless vision based motion capture also provides a potential alternative for affordable capture of human motion in a wide range of settings, and has received a great deal of attention from the computer vision and biomechanics community.

Goffredos, Seely, Carter \& Nixon [35] developed a novel 2D markerless view independent gait analysis algorithm which does not need camera calibration or preknowledge of subject pose so that it can be used for gait identification in scenarios where only a single remote camera view is presented. In this approach, information on the human silhouette and on the human body anthropometric proportions are used for the estimation of the lower limbs' pose in the image reference system with low computational cost. After the markerless motion estimation, the obtained angles' trends are corrected by the view point independent gait reconstruction algorithm proposed by the authors, that allows reconstruction of the limbs' pose in the sagittal plane. Although the proposed method is intended for identification by gait, the advantages can be utilized for elder care home gait monitoring purpose.

Saboune and Charpillet [36]developed a new approach for markerless human motion capture from conventional camera feeds. Their aim was to recover 3D positions of key points of the body that can serve for gait analysis while walking. The final analysis was designed to help doctors and caregivers detect balance disorders and predict falls in 
the elderly based on a knowledge of gait parameters. Foreground segmentation, an articulated body model and particle filtering are the basic elements of this approach. No dynamic model is used; thus this system can be described as generic and simple to implement. A modified particle filtering algorithm, which is called Interval Particle Filtering, is used to reorganize and search through the model's configurations search space in a deterministic optimal way. The Saboune-Charpillet algorithm was able to perform human movement tracking with success. Results from the treatment of a single camera feeds are shown and compared to results obtained using a marker based human motion capture system. Using a single camera hides some body parts (depending on the view angle) for a long portion of the footage and the movement of these parts cannot be evaluated fairly. Using multiple cameras would solve this problem but implicates the complexity of calibrating and adjusting a stereo vision system. Saboune and Charpillet will develop and test their multiple-camera system to analyze the gait of senior fallers during an experiment to be held in the geriatrics department of a hospital. In fact seniors will be asked to follow a specially arranged path and the gait parameters will be evaluated and analyzed in order to evaluate the pertinence of some variables.

For researchers at the Intelligent Assistive Technologies and Systems Laboratory, University of Toronto, ON, Canada, there is growing interest in vision-based technology for balance assessment. These rehabilitation engineers, Goffredo, Schmid, Conforto, \& D'Alesso [37] have developed a markerless image processing algorithm to estimate the anterior/posterior trajectory of the center of mass from video sequences obtained from commercially available systems, while standing with stable and unstable support [37]. The same laboratory served as a test bed for Allin, Beach, Mitz, \& Mahailidis [38] who 
demonstrated using a single, uncalibrated camera to measure clinically meaningful statistics of standing postural sway among an elderly balance-impaired cohort [38]. Allin and Mahailidis[39] along with Goffredo, Schmid, Conforto, Carli, Neri, \& D’Alessio [40] took a different approach in assessing balance impairment by detecting sit-to-stand strategies using web cameras. The long-term goal is to create systems that can monitor functional movements common at home in a way that reflects changes in stability and impairment. Particularly, Goffredo et al. [40] used the markerless computer vision technique to track natural elements on the human body surface. The method implements the estimate of translation, rotation, and scaling by means of a maximum likelihood approach carried out in the Gauss-Laguerre transform domain. Experimental results in terms of tracking performance are obtained by analyzing video sequences capturing the execution of the sit-to-stand task in two groups of young and elderly volunteers. The results are compared with clinical studies that used marker-based systems, and are particularly encouraging for a future extension of the approach to other motor tasks and to predict scores obtained from the physical performance batteries that are widely and regularly used by clinicians and physical therapists.

\section{c) Sensor Fusion Systems}

Existing research has shown that there is a complementary relationship between different sensing paradigms. In [41, 42, 43], the researchers demonstrated effective sensor fusion can be used to combine the strengths of ambient and wearable sensors by fusing sensory data at hardware, raw data, feature, or decision levels. The use of wearable sensors for home monitoring provides an effective means of inferring a patient's level of 
activity. However, wearable sensors have intrinsic ambiguities that prevent certain activities to be recognized accurately.

Saboune and Charpillet [36], introduced a robust framework for enhanced activity recognition by integrating an ear-worn activity recognition (e-AR) sensor with ambient blob-based vision sensors. Accelerometer information from the e-AR is fused with features extracted from the vision sensor by using a Gaussian Mixture Model Bayes classifier. Experimental results showed a significant improvement of the classification accuracy compared to the use of the e-AR sensor alone.

Stanford University's Tabar, Keshavarz, and Aghajan [42] developed a wireless sensor network employing multiple sensing and event detection modalities and distributed processing for smart home monitoring applications. Image sensing and vision-based reasoning verify and further analyze events reported by other sensors. The system dresses the growing need for independent-living groups who must have timely access to caregivers when needed. An example of sensed events is the accidental fall of anyone under as needed care. A wireless badge node acts as a bridge between the user and the network. The badge node provides user-centric event sensing functions such as detecting falls, and also provides a voice communication channel between the user and the care giving center when the system detects an alert and dials the center. The voice connection is carried over an IEEE 802.15.4 radio link between the user badge and another node in the network that acts as a modem. Using signal strength measurements, the network nodes keep track of the approximate location of the user in the monitoring environment. The network also includes wall-mounted image sensor nodes, which are triggered upon detection of a fall to analyze their field-of-view and provide the care giving center with 
further information about the user's status. A description of the developed network and several examples of the vision-based reasoning algorithm are presented in the paper.

Siciliano, Leone, Diraco, Distante, Malfatti, Gonzo, Grassi, Lombardi, Rescio, and Malcovati [43] used their Sensors and Microsystems Laboratory (SMS) at the University of Pavia, Italy to develop new technologies to support independence and engagement in elderly people living alone at home in the framework of a new UE Integrated Project. This study's aim was to develop a light technological infrastructure for integration into the homes of the elderly at reduced cost allowing the detection of critical health situations. In particular a reliable fall detector is presented with focus on the protection and assistance to the elderly in the home environment. The integrated fall detector prototype includes two different sensors: a 3D Time-of-Flight range camera and a wearable MEMS accelerometer. The devices are connected in a networked configuration with custom interface circuits to a central PC that collects and processes the information with a multithreading approach. For each sensor, an optimized algorithm for fall-detection has been developed and benchmarked on a collected multimodal database. This work is expected to lead to a multi-sensory approach employing appropriate fusion techniques which will improve system efficiency and reliability.

The sensor network under development by Skubic et. al [44 ] is also an example of sensor fusion system, which includes a passive physiological sensor network with data monitor and motion sensors, stove sensors bed sensors, and an event-driven, video sensors network that hides identifying features of the residents. The network consists of a reasoning engine that fuses sensor and video data and analyze patterns of behavioral activity; a flexible alert manager; a component for providing customization of sensor 
configuration, alert specification, and data access for each resident; and a database server and we interface that provides interactive retrieval and visualization of the sensor data. The sensor network is currently being developed and tested in TigerPlace. TigerPlace is an independent living apartment complex for seniors in Columbia, Missouri. A joint venture between MU's Sinclair School of Nursing and Americare Systems Inc., TigerPlace is one of four projects granted state approval to operate under the "aging in place" model of care giving. [45].

\subsection{Three Dimensional Voxel Reconstruction from Silhouettes}

a) Background subtraction techniques

The ultimate goal for the technology proposed in this dissertation is to monitor an elderly person's gait in his normal daily environment; therefore, privacy needs to be addressed. Instead of using raw images, we perform a silhouette extraction, namely, segmenting the human body from the background with the camera at a fixed location at the initial stage in the analysis. Before silhouette extraction can occur, an accurate background model must be acquired. The background is defined as any non-human, static object. After the background model is initialized, regions in subsequent images with significantly different characteristics from the background are considered as foreground objects. Areas classified as background are also used to update the background model. A large number of background adaptation and foreground segmentation algorithms have been proposed. A short list of the most valuable includes Temporal Difference, Mean and Threshold, Gaussian Mixture Model [46], Eigen Backgrounds [47], and Wallflower [48]. 
Fused texture and color features developed by MU CERT team are used for background subtraction for this study [49].

b) Camera calibration

Before processing any data in $3 \mathrm{D}$ space, calibration must be performed to determine the world coordinates and orientations for all cameras. Camera calibration is the process of finding the true parameters of the camera that produced a given photograph or video. Usually, camera parameters are represented in a $3 \times 4$ matrix called the camera matrix. The world and image coordinates systems are illustrates in Fig.2.1.
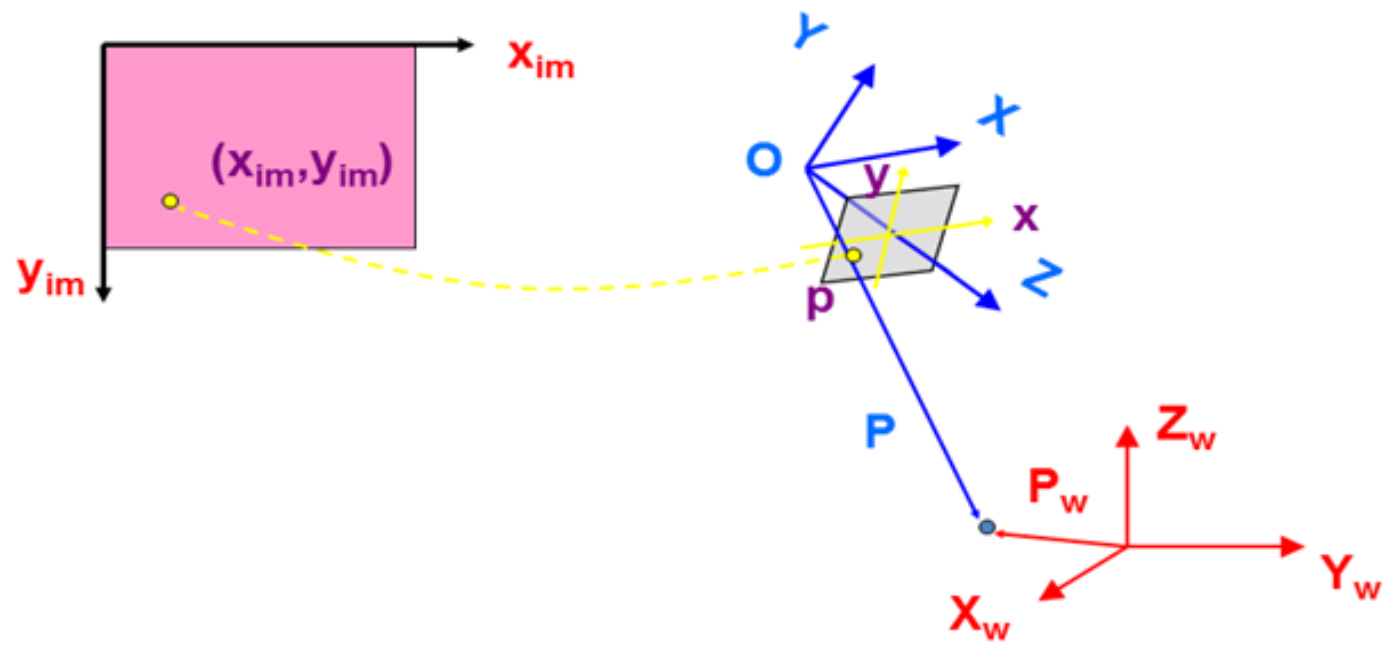

Fig.2.1 World and image coordinates systems.

Here is a brief review of the derivation of the perspective projection equations.

- World to Camera

Camera: $P=(X, Y, Z)^{T}$

World: $P_{w}=\left(X_{w}, Y_{w}, Z_{w}\right)^{T}$

Transform: R,T 


$$
\begin{aligned}
& {\left[\begin{array}{c}
X \\
Y \\
Z \\
1
\end{array}\right]=\left[\begin{array}{cc}
R & T \\
\overline{0} & 1
\end{array}\right] \cdot\left[\begin{array}{c}
X_{w} \\
Y_{w} \\
Z_{w} \\
1
\end{array}\right]} \\
& R=\left[\begin{array}{l}
r_{1} \\
r_{2} \\
r_{3}
\end{array}\right] \quad T=\left[\begin{array}{l}
t_{x} \\
t_{y} \\
t_{z}
\end{array}\right]
\end{aligned}
$$

- Camera to Image

Camera: $P=(X, Y, Z)^{T}$

Image: $p=(x, y)^{T}$

$$
\begin{aligned}
& \left(\begin{array}{ll}
x & y
\end{array}\right)=\left(\begin{array}{lll}
-f \frac{X}{Z} & -f \frac{Y}{Z}
\end{array}\right) \text { or } \\
& {\left[\begin{array}{l}
x \\
y \\
s
\end{array}\right]=\left[\begin{array}{cccc}
-f & 0 & 0 & 0 \\
0 & -f & 0 & 0 \\
0 & 0 & 1 & 0
\end{array}\right] \cdot\left[\begin{array}{c}
X \\
Y \\
Z \\
1
\end{array}\right]}
\end{aligned}
$$

- Image to Frame

Neglecting distortion

Frame: $\left(x_{i m}, y_{i m}\right)^{T}$

$$
\left[\begin{array}{c}
x_{i m} \\
y_{i m} \\
s
\end{array}\right]=\left[\begin{array}{ccc}
k_{x} & 0 & o_{x} \\
0 & k_{y} & o_{y} \\
0 & 0 & 1
\end{array}\right] \cdot\left[\begin{array}{l}
x \\
y \\
s
\end{array}\right]
$$

Put all above equations together, we can get the world to frame transformation.

- World to Frame 


$$
\begin{aligned}
& {\left[\begin{array}{c}
x_{i m} \\
y_{i m} \\
s
\end{array}\right]=\left[\begin{array}{ccc}
k_{x} & 0 & o_{x} \\
0 & k_{y} & o_{y} \\
0 & 0 & 1
\end{array}\right] \cdot\left[\begin{array}{cccc}
-f & 0 & 0 & 0 \\
0 & -f & 0 & 0 \\
0 & 0 & 1 & 0
\end{array}\right]\left[\begin{array}{cc}
R & T \\
\overline{0} & 1
\end{array}\right] \cdot\left[\begin{array}{c}
X_{w} \\
Y_{w} \\
Z_{w} \\
1
\end{array}\right]} \\
& {\left[\begin{array}{c}
x_{i m} \\
y_{i m} \\
s
\end{array}\right]=\left[\begin{array}{cccc}
-f k_{x} & 0 & o_{x} & 0 \\
0 & -f k_{y} & o_{x} & 0 \\
0 & 0 & 1 & 0
\end{array}\right]\left[\begin{array}{cc}
R & T \\
\overline{0} & 1
\end{array}\right] \cdot\left[\begin{array}{c}
X_{w} \\
Y_{w} \\
Z_{w} \\
1
\end{array}\right]=\left[\begin{array}{cccc}
-\alpha_{x} & 0 & o_{x} & 0 \\
0 & -\alpha_{y} & o_{x} & 0 \\
0 & 0 & 1 & 0
\end{array}\right]\left[\begin{array}{cc}
R & T \\
0 & 1
\end{array}\right] \cdot\left[\begin{array}{c}
X_{w} \\
Y_{w} \\
Z_{w} \\
1
\end{array}\right]} \\
& \mathrm{l}^{1} \\
& \text { Intrinsic Extrinsic } \\
& \text { Parameters Parameters } \\
& \text { Add skew factor } \gamma \text {, the intrinsic matrix is: }\left[\begin{array}{cccc}
-\alpha_{x} & \gamma & o_{x} & 0 \\
0 & -\alpha_{y} & o_{x} & 0 \\
0 & 0 & 1 & 0
\end{array}\right]
\end{aligned}
$$

The intrinsic matrix contains 5 intrinsic parameters. These parameters encompass focal length, image format, and principal point. The parameters $\alpha_{x}=f \cdot k_{x}$ and $\alpha_{y}=f \cdot k_{y}$ represent focal length in terms of pixels, where $k_{x}$ and $k_{y}$ are the scale factors relating pixels to distance. $\gamma$ represents the skew coefficient between the $\mathrm{x}$ and the $\mathrm{y}$ axis, and is often $0 . o_{x}$ and $o_{y}$ represent the principal point, which would be ideally in the center of the image.

$\mathrm{R}$ and $\mathrm{T}$ are the extrinsic parameters which denote the coordinate system transformations from 3D world coordinates to 3D camera coordinates.

Anderson et al. [50] worked with a voxel resolution of $5 \times 5 \times 5$ inches. With such a coarse resolution, the camera calibration was not a significant issue. Here, the camera calibration was improved to yield more accurate voxel reconstructions. An intrinsic 
model of each camera is estimated using the Camera Calibration Toolbox from [51]. Given intrinsic models of the cameras, for a given camera configuration, the 6 DOF (6 Degrees of Freedom including rotation and translation) location (position and orientation) of each camera in the environment is computed independently using correspondences between a set of 5 or more measured 3D locations in the environment and pixels in the camera image. The 6 DOF location of each camera is then optimized such that the pixel projection error of the set of $3 \mathrm{D}$ points is minimized. Given the optimized location, along with the intrinsic model, the calibrated view vector of each pixel in each camera can be determined for the purpose of silhouette back projection. This calibration procedure results in significantly more accurate voxel person reconstructions as compared to the previous technique.

c) Voxel reconstruction

This research benefited from the University of Missouri-Columbia Center for Eldercare and Rehabilitation Technology directed by this dissertation's advisor, Dr. Marjorie Skubic. The MU Eldercare Rehab Team's (CERT) three-dimensional human model, called voxel person, described and used in $[50,54]$, is constructed in voxel (volume element) space by back projecting silhouettes from multiple camera views. A voxel is a three-dimensional volume (a non-overlapping cube) resulting from the discretization of the environment, similar to how pixels discretize an image. Voxels are specified according to their center location, and widths. Generally, the widths are the same for all dimensions. Here, the voxel resolution is $1 \mathrm{x} 1 \mathrm{x} 1 \mathrm{inch}$. The voxel is associated with a number indicating inclusion/exclusion of the element in a particular set (e.g. a threedimensional object representing the human). As part of the MU CERT, F. Wang worked with team member E. Stone using camera calibration to determine a list of voxels for each 
pixel representing the voxels visible to that pixel. There are two general approaches to modeling a scene using voxels, additive and subtractive. Additive modeling starts with an empty set (scene) and adds elements (voxels) to that set as they are found. Respectively, subtractive modeling starts initially with the full voxel space, and elements (voxels) not visible are removed. The basics of voxel reconstruction are illustrated in Fig.2.2. And Fig.2.3 illustrated an example of silhouettes extraction, and voxel person reconstruction used in this study.

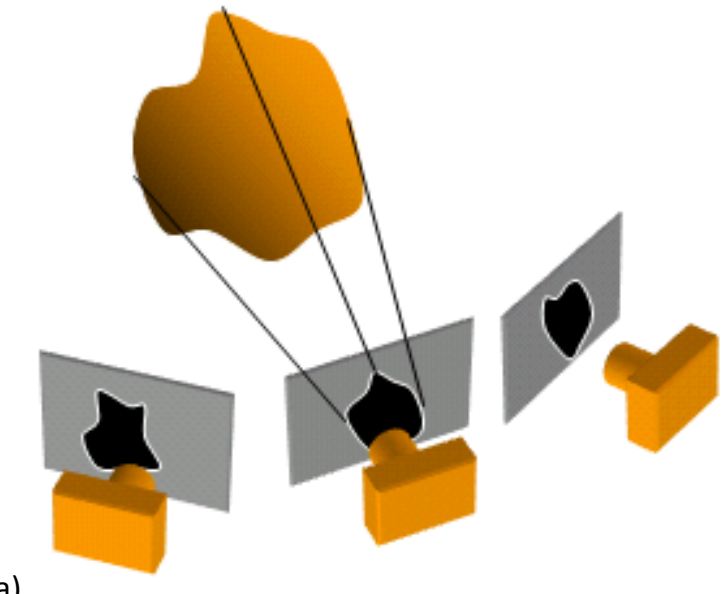

(a)

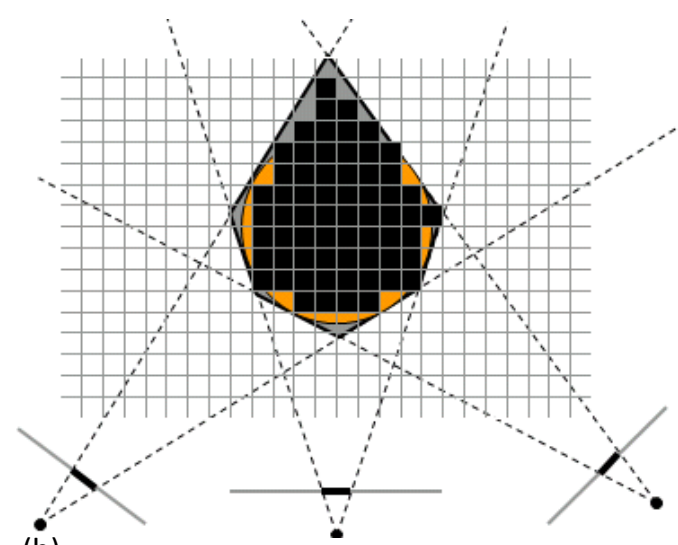

(b)

Fig.2.2 (a) Backproject each silhouette and intersect backprojected volumes (b) Color voxel black if on silhouette in every image 

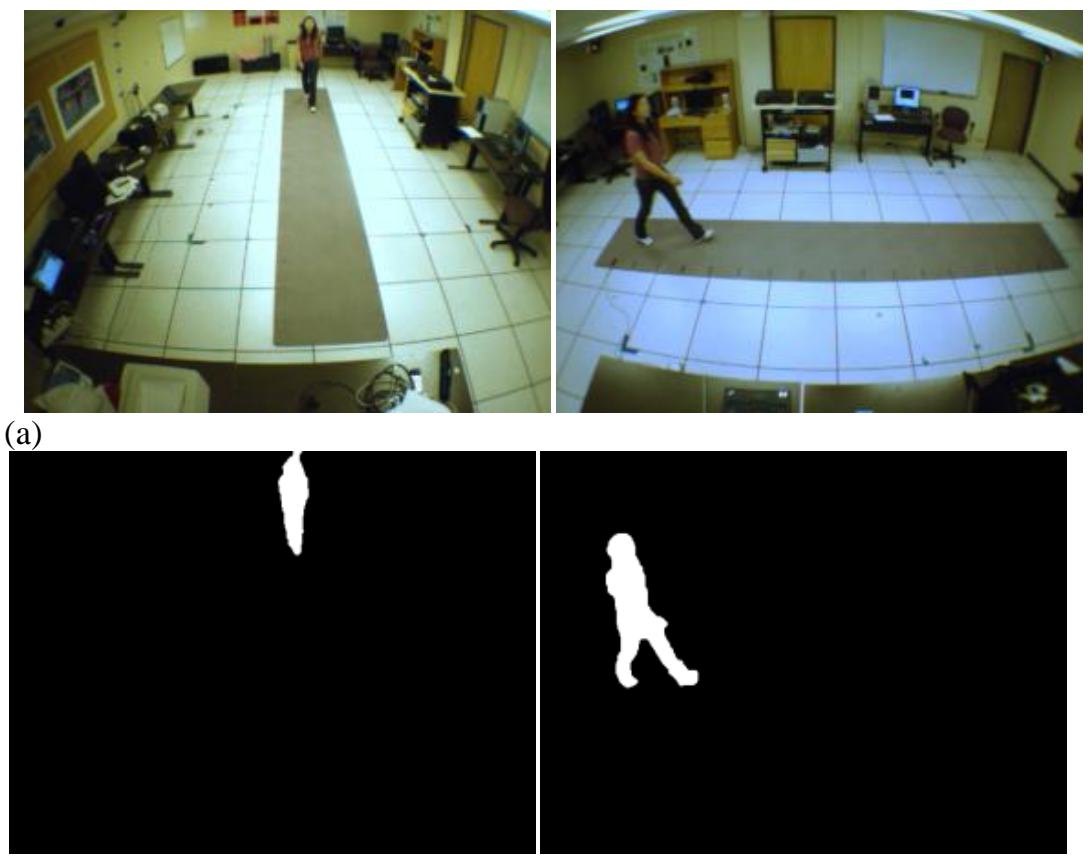

(b)

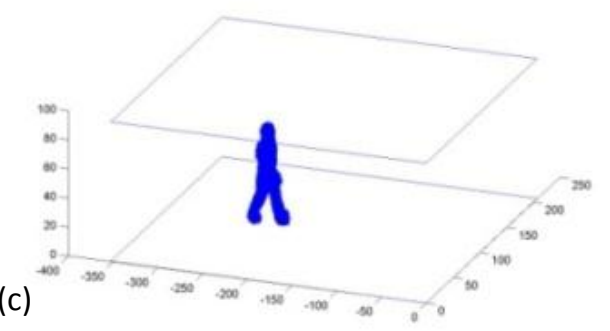

Fig.2.3 (a) Two raw camera images monitoring the same scene. (b) Human silhouettes (c) The reconstructed three-dimensional voxel person. 


\section{Chapter 3. Methodology}

\subsection{System Overview}

The high level system overview of the proposed motion analysis system is illustrated in Fig.3.1. The blue items are the focus of this dissertation, and their methodology and algorithms are presented in the following sections. Each component as well as the system as a whole has been validated and tested with in-lab testing, and $1^{\text {st }}$ and $2^{\text {nd }}$ round TigerPlace testing.

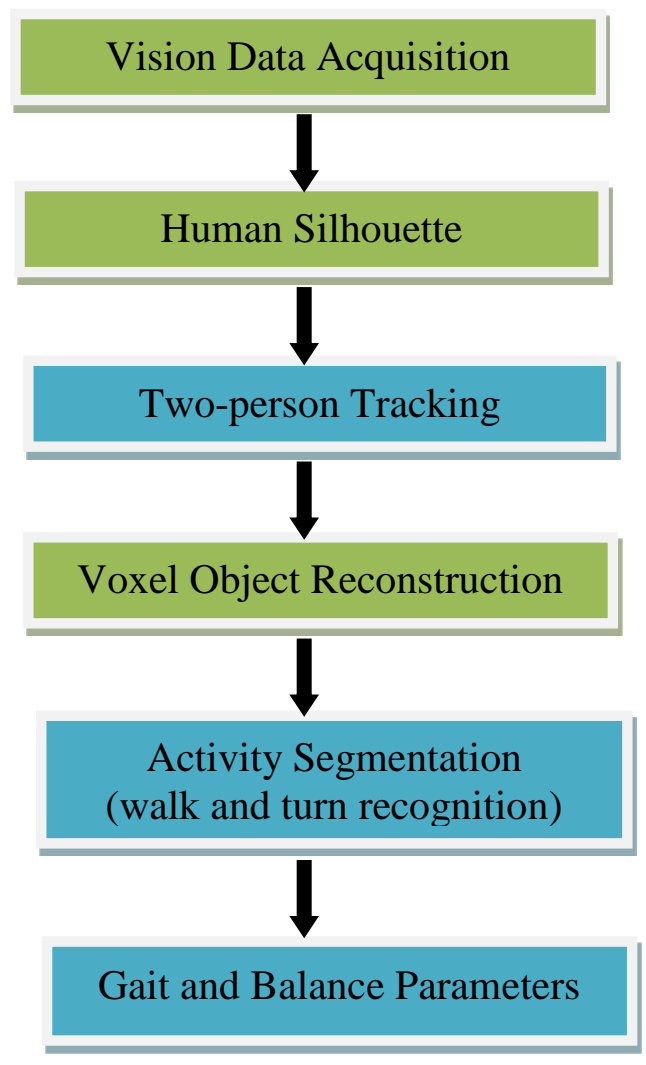

Fig.3.1 High level system overview of the proposed motion analysis system for gait and balance assessment 


\subsection{Two-Person Scenario Tracking}

This research involves two-person scenarios using two cameras. In order to reconstruct the voxel person properly, it was necessary to track and correspond the two persons from the two cameras. Thus, the tracking involves two different modes:

- Single Camera Tracking: matching subjects between consecutive frames.

- Multiple Camera Cooperative Tracking: matching subjects across cameras.

F. Wang worked on the single camera tracking of the two-person scenarios. To track people with a single camera, the system performs two major tasks: detecting the moving people and matching the subjects between consecutive frames.

Detecting moving people is currently done through silhouettes extraction, and possibly in the future with the help from the people finder algorithm developed in MU's ERT research group to improve its accuracy.

To reliably track the detected people, the tracking algorithm uses apparent color and motion continuity. Once the silhouettes' foreground is properly pre-processed, in almost all cases, only humans are shown as foreground. For each input silhouette image, the tracking algorithm starts with connected components analysis and then calculates normalized color histogram for each connected object. This data is then compared with the color histogram model to find the closest match. The match is performed using Bhattacharyya distance. The Bhattacharyya measure or coefficient is a divergence-type measure between distributions, defined as:

$$
\rho\left(p, p^{\prime}\right)=\sum_{i=1}^{n} \sqrt{p(i) p^{\prime}(i)}
$$


where $p(i)$ and $p^{\prime}(i)$ represent two multi-dimensional vectors. The Bhattacharyya measure has a simple geometric interpretation as the cosine of the angle between the $\mathrm{N}$-dimensional vectors If the two distribution are identical, we have $\rho=1$.

Primary Advantage and Disadvantage: Color histogram is a representation of the distribution of colors in an image, derived by counting the number of pixels of each of given set of color ranges in a typically 3D color space. The key advantage to using histograms for classification is simplicity, due to the fact that pixels are relatively invariant with translation and rotation about the viewing axis, varying only slowly with the angle of view. The main drawback or disadvantage is that the representation relies on the color of the object being studied, ignoring its shape and texture.

Due to the fact that the people in the scenario change their appearance, such as wearing a jacket, the model color histogram update is necessary. However, if a model color histogram is ever mistakenly updated, it cannot be easily recovered. Therefore, F. Wang used motion continuity as a rule for the color histogram updating based on the assumption that the subject should not move beyond a certain set threshold in consecutive frames. There are cases when two persons were close enough that their silhouettes connected together. In this case, the algorithm also doesn't update the color histogram model. Also in this case, the algorithm cannot distinguish the two persons from a joint connected object, and would keep the silhouette unchanged. It might still be possible to construct two separate voxel persons if the silhouettes are well separated in the other camera view. 
Algorithm 1: Two-person tracking

Input: RGB raw images Img_r, Silhouette images Img_s, number of persons $N$ Output: Labeled images

Initialize RGB color histogram distribution $H 1$ for person1, H2 for person 2 as zeros

For each frame $t$

Read in img_r;

Read in img_s;

Compute RGB color histogram on each foreground object;

Compare color histogram of foreground objects with $\mathrm{H} 1, \mathrm{H} 2$ by computing Bhattacharyya coefficient;

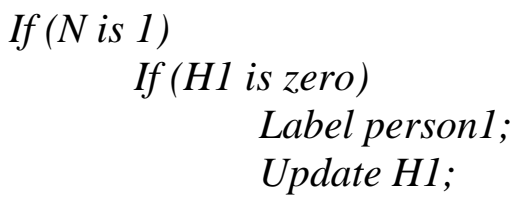

Else

Find one best match to $\mathrm{H} 1$ or $\mathrm{H} 2$, label accordingly

If $(N$ is 2$)$

If ( $\mathrm{H} 1$ and $\mathrm{H} 2$ are both zero)

Label randomly; update $H 1$ and $H 2$;

Elseif (H2 is zero and number of foreground object is 2)

Find best match for personl and label the other foreground object as person2;

Update $\mathrm{H} 2$;

Elseif (number of foreground object is 1)

Output original silhouette image;

Else

Find the maximum Bhattacharyya coefficient, and label this foreground object to its corresponding person;

Find best match for the other person;

Test the labeled foreground human centroid to the previous fame, and updated their color histogram if needed; 
The tracking algorithm can be described as following: Compare the color histogram of each foreground object in the image with the model color histogram using the Bhattacharyya coefficient, and find the closest match; then update the color histogram model if the new matched candidate centroid is within a certain distance (preset number of pixels threshold) from the previous frame. The algorithm is based on the assumption that in most cases, the foreground objects in silhouette images are human, at least when the color histogram model is first updated from initial zero values. In addition, the number of persons in the image needs to be known as a ground truth input information. The number of persons may be input from another component of the system, such as a human detector [52].

The algorithm has been tested with the first data set collected in TigerPlace. The performance is satisfactory for next stage processing when the silhouettes are in good quality. Fig.3.2 shows an example of tracking results where the resident changed appearance. 

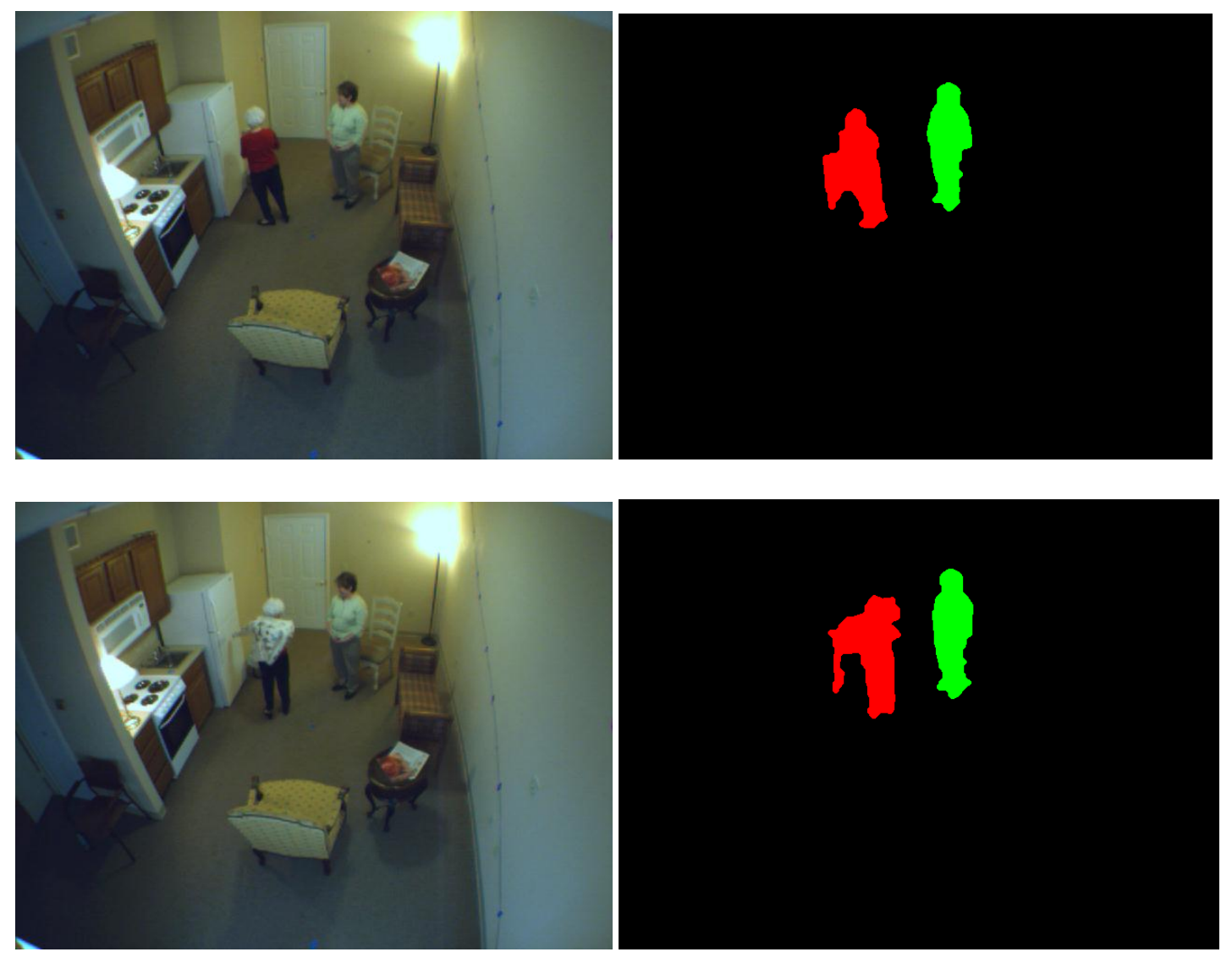

Fig.3.2 Original images and tracking silhouettes illustration. In silhouette, red is for the resident, and green is for visitor.

\subsection{Activity Segmentation}

When presented with large amount of video information of a person's daily activities, it is necessary to locate the images suitable for gait analysis. Proper activity segment is therefore necessary and important for an accurate and valid gait analysis. Due to the simple nature of the scripted scenario, the videos are automatically segmented using velocity values and velocity vector angles.

The velocity overview is shown in Fig.3.3 for ID4 Test 2. The velocity data then goes through curve smoothing (cubic spline) as shown in Fig.3.3 (b). Based on the smoothed 
velocity data, image frames that are above threshold of $25 \mathrm{~cm} / \mathrm{s}$ and last more than 15 frames (3 seconds) are selected as gait analysis candidates.
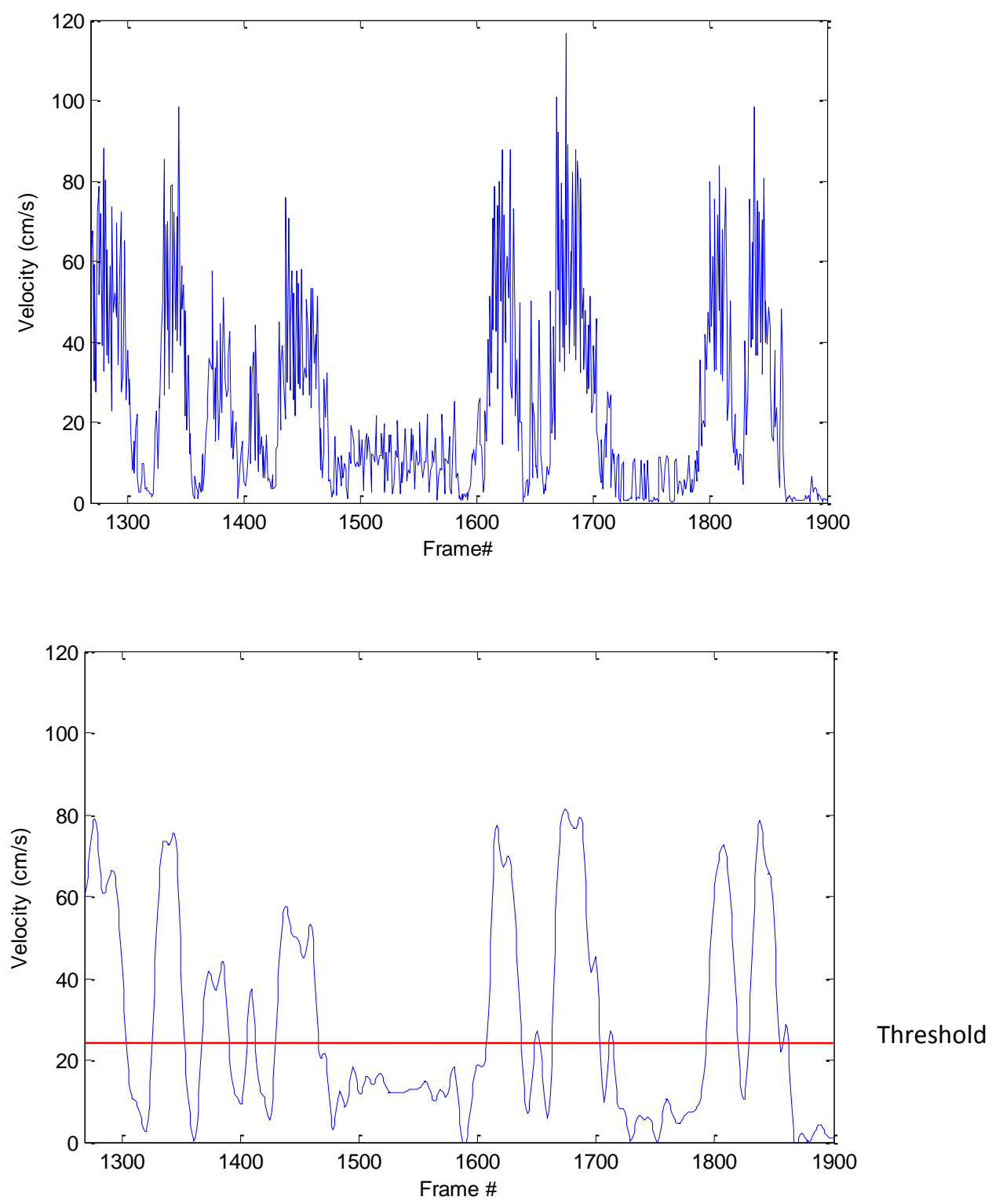

Fig.3.3 ID4 Test 2 velocity overview (a)velocity (b)cubic spline smoothing velocity used to segment walk 
As for the occlusion which is a common problem in computer vision, it should be pointed out that unlike a fall detection system, it's not necessary for the gait assessment system to perform gait analysis $24 / 7$. There is no immediate danger if the system loses track of a person for a short period of time. Instead the key lies in the capability to segment out the images that are suitable for gait analysis. The possible segment criteria include 2-D shape analysis, voxel volume analysis, and foot area analysis etc. In Fig.3.4 the project foot area and voxel person volumes are illustrated using ID5 run1 from $2^{\text {nd }}$ round TigerPlace dataset. The two red circled regions in Fig.3.4 (a) are the two places where the projected foot area decreased to zero or near zero. The corresponding images and voxel person images are illustrated in Fig.3.4 (c) and (d) where the person's lower body is partially occluded. In addition, when the voxel person's volume is over-sized, such as shown in Fig.3.4 (e) and (f) (peak volume in Fig.3.4 (b)), which at this case are the results of the voxel person being located in a bad back-projection region. Oversized subject areas (which could include a large person and his auxiliary walker, oxygen unit, etc.) are also not suitable for gait analysis. However, more studies are necessary to determine the proper volume or foot area values as a threshold for image selection. 

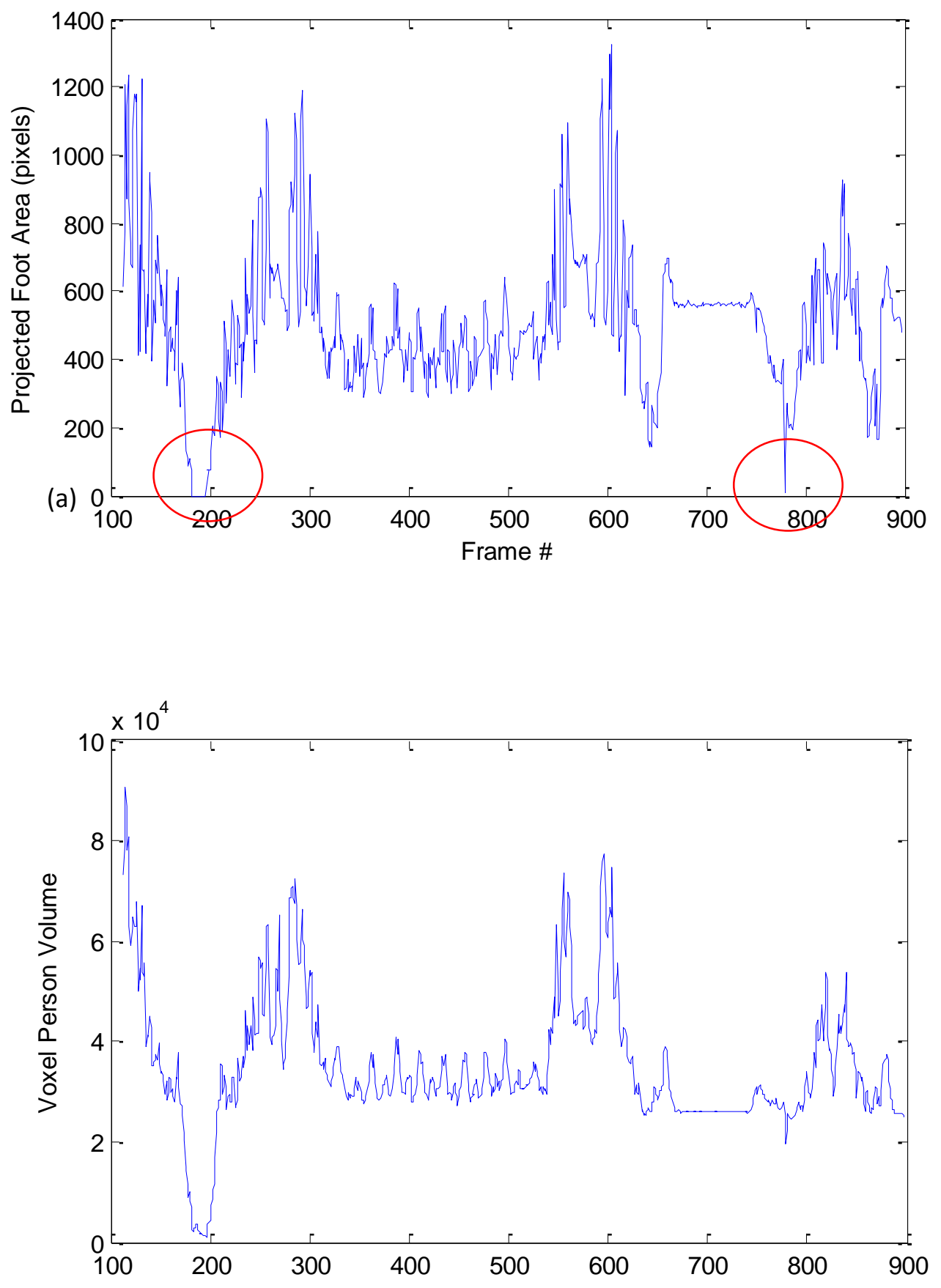

(b)

Frame \# 

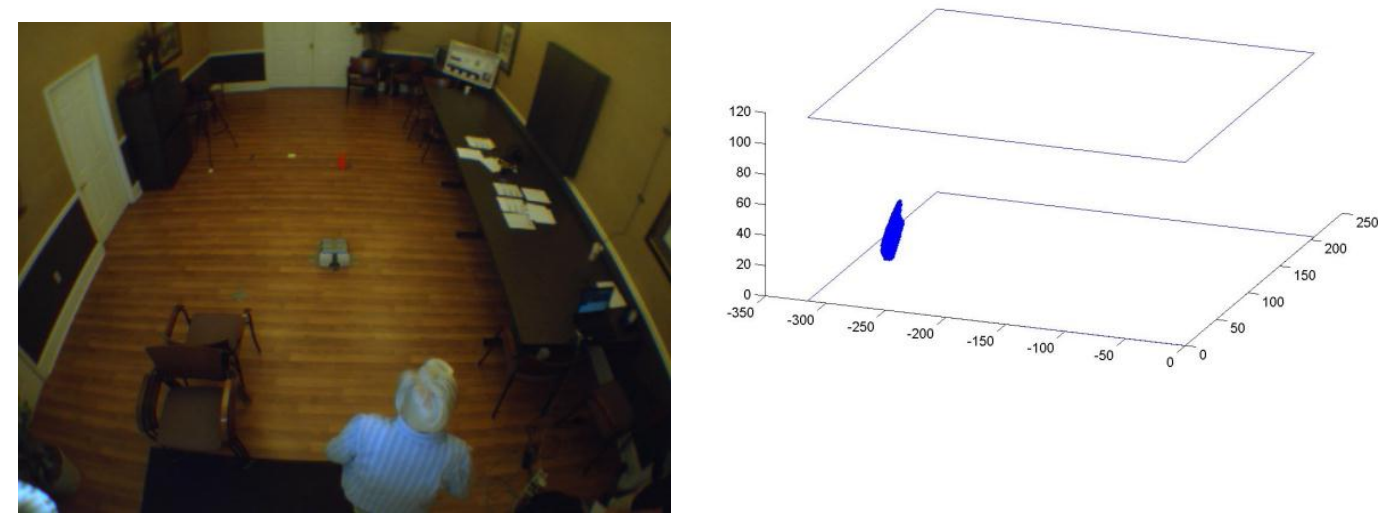

(c) Frame 200
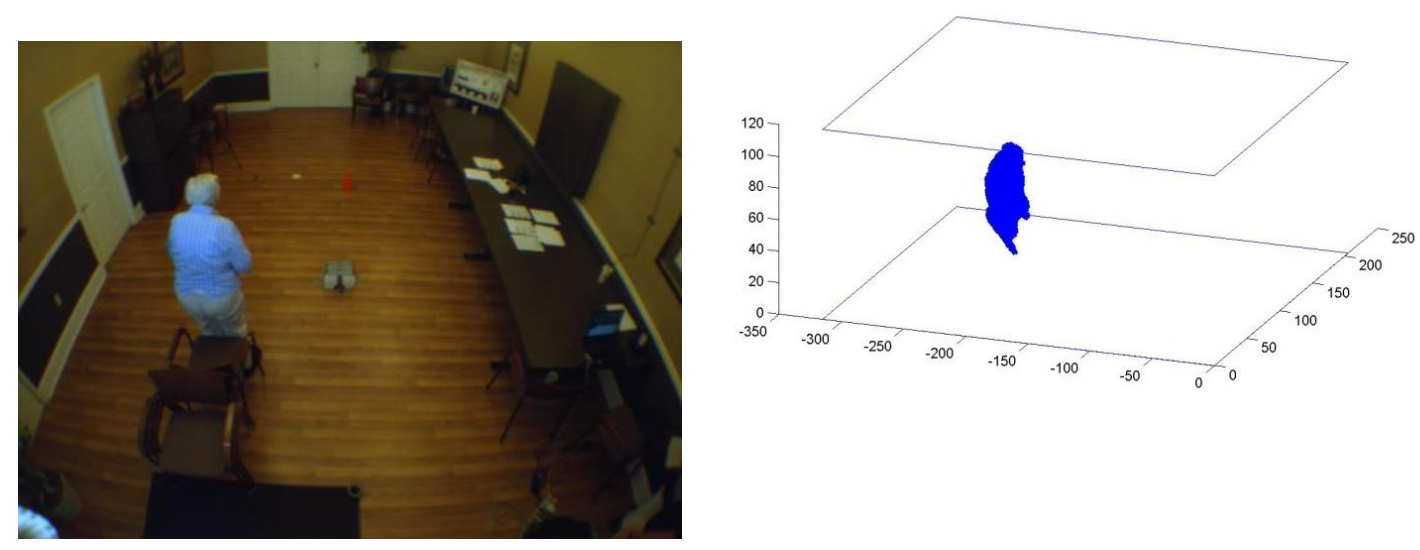

(d) Frame 779
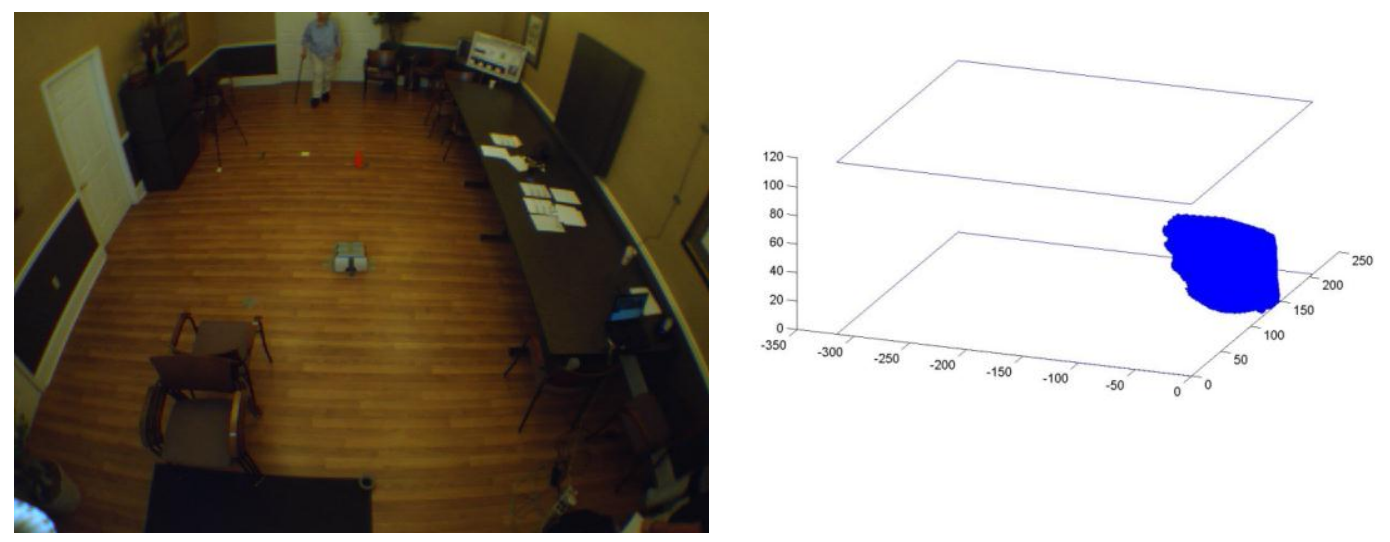

(e) Frame 115 

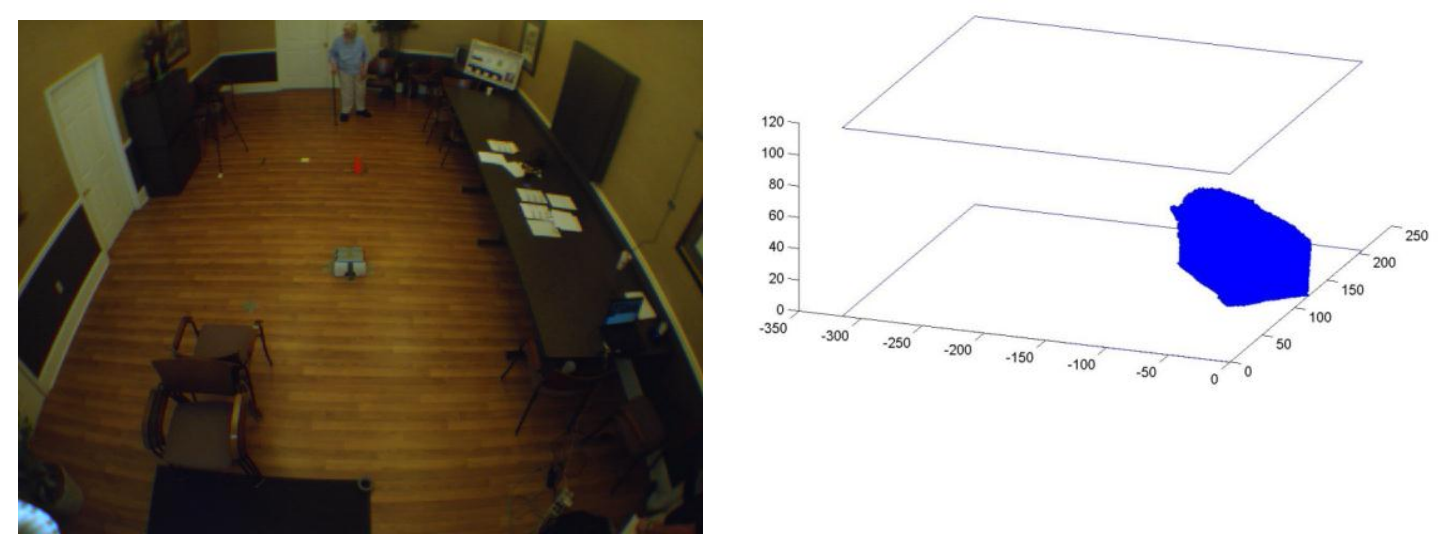

(f) Frame 286

Fig.3.4 Illustration of frame segmentation based foot area size and voxel person volume using ID5 run 1 from $2^{\text {nd }}$ round TigerPlace dataset as an example (a) Foot area size (b) Voxel person volume (d) Frame 200, lower body occluded (e) Frame 779, lower body occluded (f) Frame 115, bad backprojection region (f) Frame 286, bad backprojection region

Algorithm 2: Activity segmentation

Input: Voxel $3 D$ centroid data, threshold for velocity $T_{v}$, threshold for voxel foot area $T_{f}$, threshold for number of consecutive frames $T_{c}$;

Output: Walking frames

Calculate velocity, and apply curve smoothing;

Calculate velocity vector angle as Equation (7), and apply curve smoothing;

Eliminate bad frames in which voxel area lower than 5 inches(foot area) is below threshold $T_{f}$;

Select frames above threshold velocity $T_{v}$;

Select walking frames above $T_{c}$; 


\subsection{Extraction of Gait Parameters}

\subsubsection{Gait Parameters from Cameras}

To validate our method, we used GAITRite mat and Vicon motion capture system for ground truth. Gait Parameters need to be extracted for both the web camera and Vicon systems and compared with the GAITRite results. The extraction method for the Vicon system is presented in Section 3.4.2.

In order to get an accurate comparison, the GAITRite system (available commercially from CIR Systems Inc.) and its measurement definitions are as follows. The GAITRite system used in the experiment is an electronic walkway with an effective length of 16 feet $(4.9 \mathrm{~m})$. The walkway comprises a series of sensor pads that are inserted in a grid. The sensors are placed $1.27 \mathrm{~cm}$ apart (total of 18,432 sensors) and are activated by mechanical pressure. Footfall data from the activated sensors are collected by a series of on-board processors and transferred to the computer through a serial port. The sampling rate of the system is $120 \mathrm{~Hz}$. The validity of GAITRite system's measurement of gait parameters was validated by Webster, Wittwer and Feller [53] at the Musculoskeletal Research Centre, La Trobe University, Melbourne, Australia (2004). The gait parameter definitions used in the GAITRite ${ }^{\circledR}$ instrumented walkway system are listed below.

Walking speed: Distance traveled divided by the ambulation time. The ambulation time is defined as the time elapsed between first contact of one foot and the last footfall.

Step time: Time elapsed from first contact of one foot to the first contact of the opposite foot.

Step length: The step length of the right foot is defined as the distance between the center of the left foot to the center of the right foot along the line of progression. 
In this project, unless specified, the step time and step length are the averages of the right and left feet.

The gait parameters are extracted for the web camera system based on the definitions above in order to compare with results obtained from GAITRite. The extraction methodology for the camera system, however, does not depend on footfalls, but is approximated using the voxel person centroid and distance between the two feet. This research was first presented by this researcher at an IEEE Engineering in Medicine and Biology Society Conference in 2009 [54].

i) Walking speed

The voxel person centroid $\boldsymbol{C}$ is the average of all the voxels locations $\vec{V}$ :

$$
C=\operatorname{average}(v)
$$

The centroid represents the 3D location of the person at a given time. The distance $D$ that a person traveled in 2D space is approximated by adding up the distance the centroid location moved at each frame. Walking speed $V$, therefore, can be calculated based on the distance traveled divided by time calculated from the frame rate $f(5 \mathrm{fps})$ and the number of frames $F$.

$$
V=D * f / F
$$

\section{ii) Step Time}

The voxels with a height below 4 inches from the ground plane are used to capture foot motion. They are projected onto the 2D space as shown in Fig.3. 5. The solid line represents the length from the front of one foot to the end of the other foot. It is projected along the walking direction. The walking direction is obtained from the voxel person 
centroid in consecutive frames. As illustrated in Fig.3. 5, this length alternatively expands (shown as peaks) and contracts (shown as valleys) over time as the person's feet spread and close during the gait cycle. The number of steps $S$ is obtained directly from the number of peaks representing the number of gait cycles (Fig.3. 5c). The average step time $T$ is then calculated as the total time divided by the number of steps.

$$
T=(F / f) / S
$$

iii) Step Length

The average step length $L$ is then calculated as:

$$
L=D / S=V * T
$$

\section{iv) Gait Asymmetry}

The body is usually symmetrical during walking when healthy. Step length, cadence, torso movement, and ankle, knee, hip, and pelvis motion are equal on the right and left sides. Loss of symmetry of motion and timing between right and left side usually indicates a disorder [55]. Gait asymmetry has also been found to be associated with elderly fallers [56].It is therefore important to be able to differentiate the right and left foot for gait analysis. 

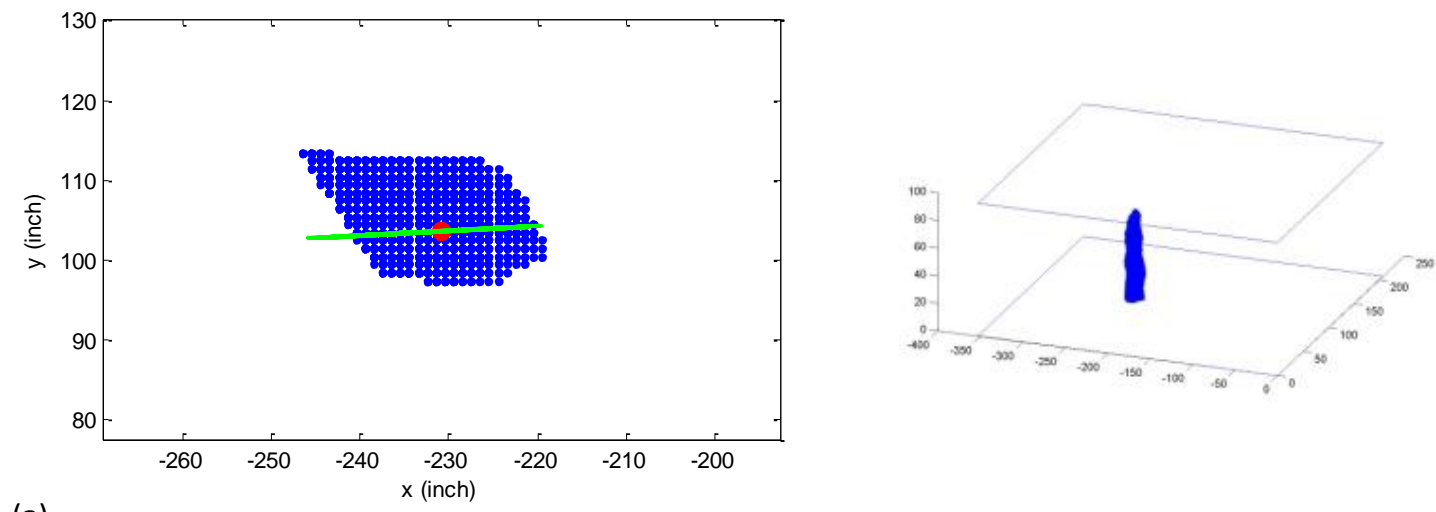

(a)
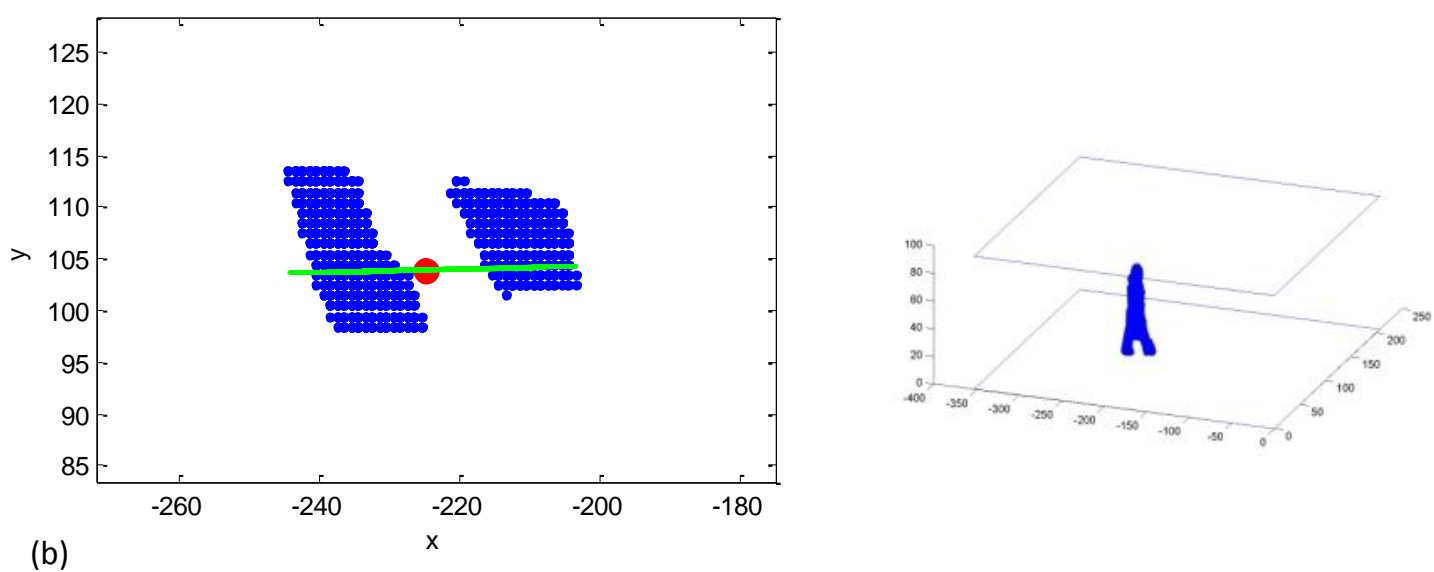

(b)

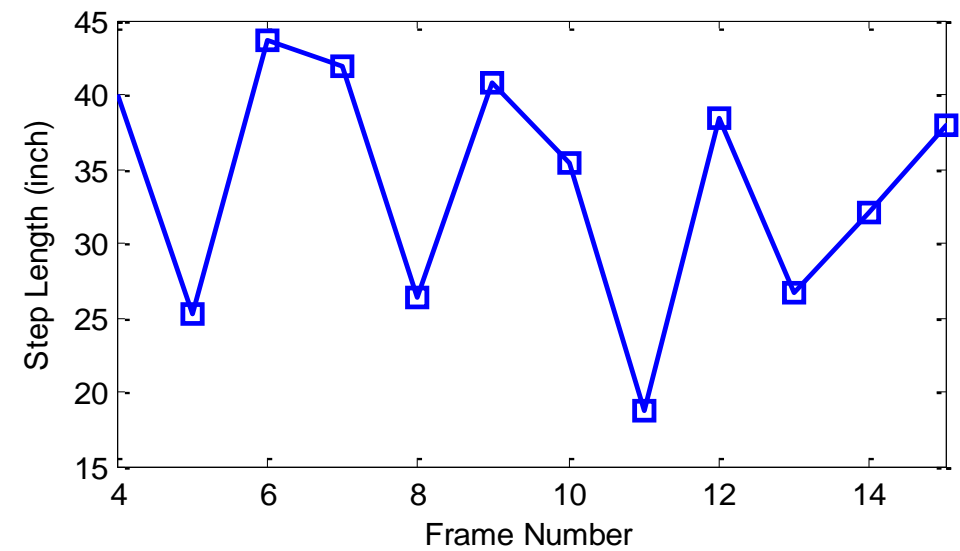

Fig.3. 5 (a) A projection of a valley frame, i.e., feet are together (b) A projection of a peak frame, i.e., feet are separated (c) Step length variation at different frames. 
In terms of step length, the difference in the right and left step length can be obtained by calculating the length for the right and left foot individually and then finding the difference between the two. As illustrated in Fig.3, right and left feet are differentiated using body centroid information. In Fig.3.6(a), the voxel person body centroid is projected onto $2 \mathrm{D}$ ground plane during a walk. The red circle points are the $2 \mathrm{D}$ positions that have been identified as double support (both feet on the ground) when the length between the two feet is at peak values as seen in Fig.3. 5c; the black x points are for single support. One vector is formed by connecting two consecutive double support points, and the other vector is formed by connecting one double support point with a single support point in a later frame. The angle is then calculated between the two vectors. As shown in Fig.3.6 (b), the negative angles are associated with right foot frames, and the positive angles are associated with left foot frames.

Under the current voxel resolution (1 inch), subtle differences between the two feet cannot be detected accurately. Asymmetry in terms of step time can be calculated in a similar way; however, its accuracy is limited by the frame rate of the camera, which was 5 frames per second in the experiments described here. 

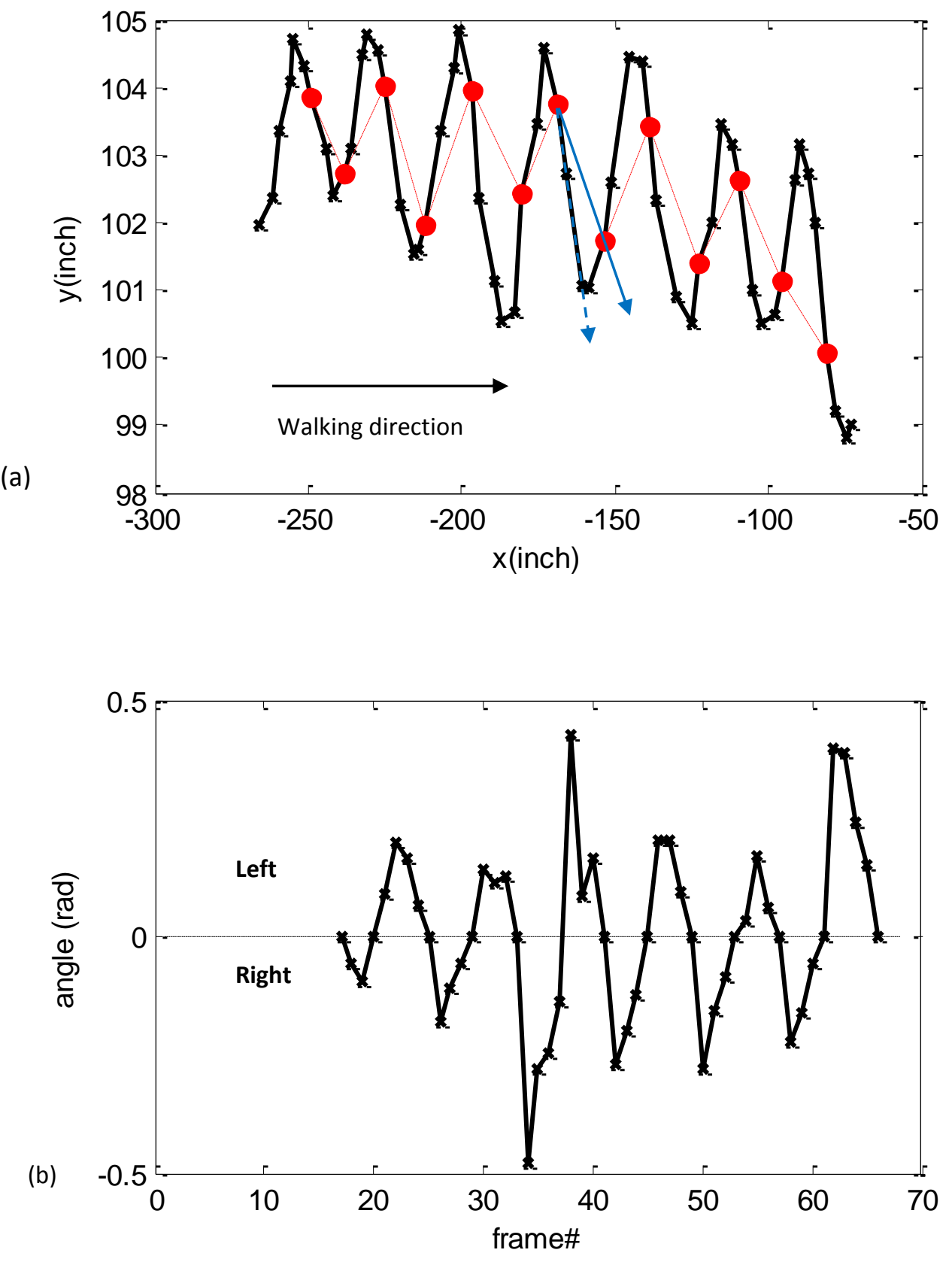

Fig.3.6 Voxel person body centroid projected in 2D space during a walk. Red circle: double support, Blue cross: right/left foot single support. Angles between red and blue arrows are extracted, as shown in b. (b) Angle values. Negative for right foot, positive for left foot. 


\section{v) Cane Removal}

As mentioned earlier, 4 out of the 10 participants recruited use a cane, even though there is no preference or consideration during the recruitment regarding the use of a cane. Due to the fact that canes are very commonly used by elderly, it is necessary to make sure the algorithm works well in these cases. As seen in Fig.3.7(a) frame 780, the cane is in front of the resident feet, and projected on the ground plane, causing inaccurate step length calculation. The connected component labeling algorithm is used to get rid of small size objects.
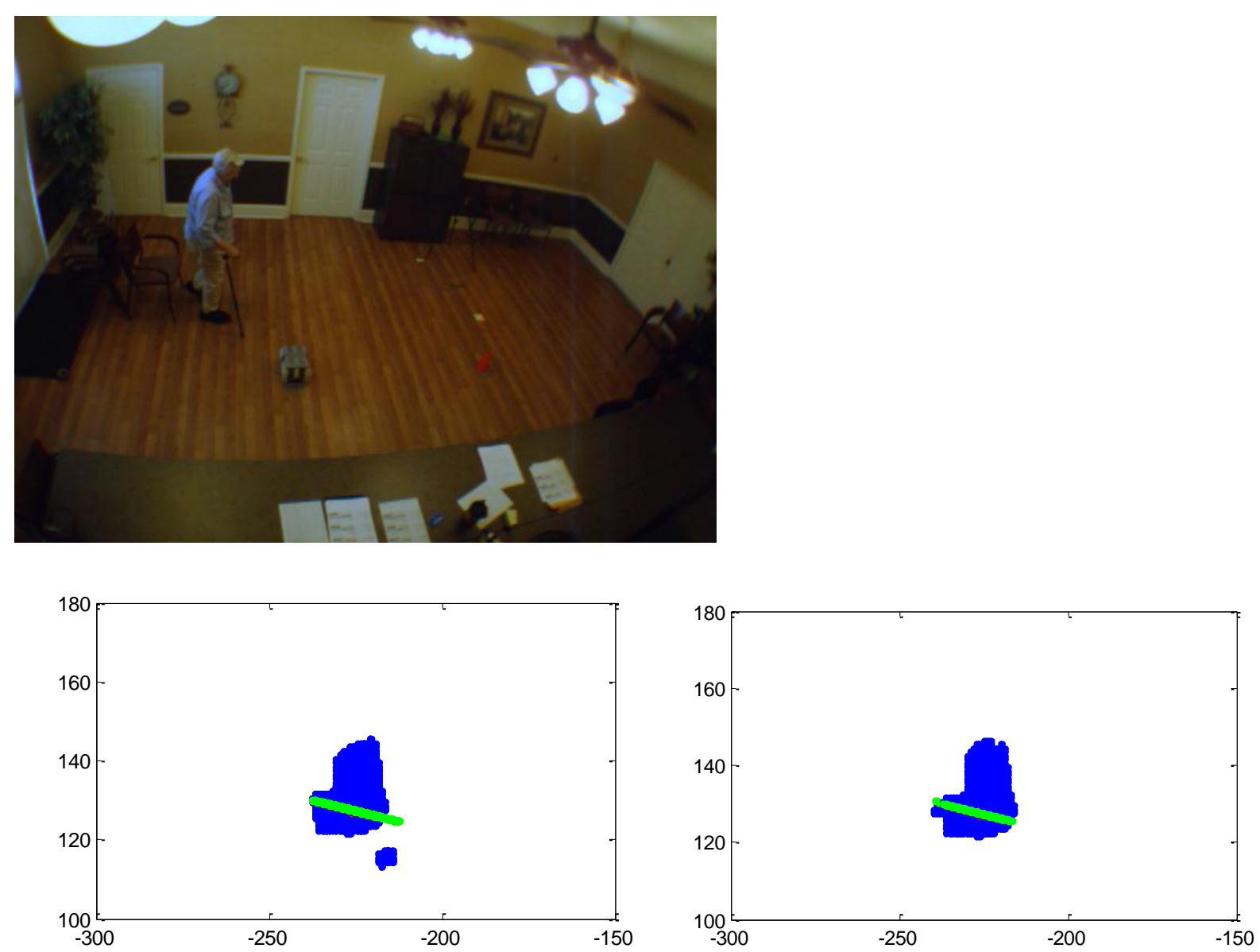

(a) Frame 780 

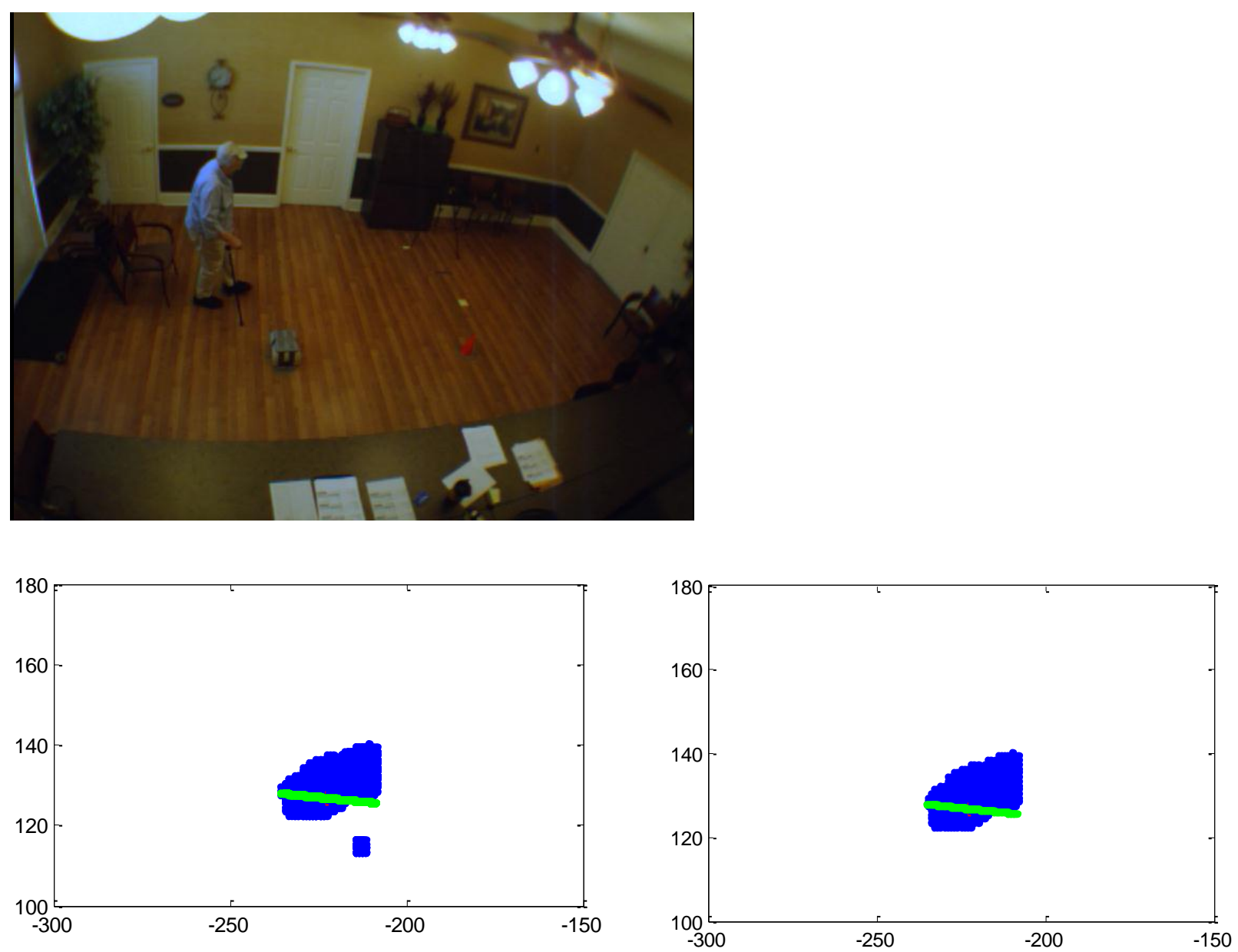

(b) Frame 781

Fig.3.7 Illustration of the cane for ID5Run1frame 780-781: ground projection of foot area before and after cane removal. 
Algorithm 3: Gait parameters extraction (velocity, step time and step length)

Input: Voxel 3D position data

Output: Gait parameters

For each frame $t$

\{

Read in voxel object $3 D$ position data;

Compute centroid of the $3 D$ object;

Obtain voxel located below 5 inch to represent feet;

Perform cane removal;

Calculate walking direction using centroid from $t-1$ to $t+1$ frame;

Project the $2 D$ feet coordinates onto the walking direction, and calculate the projected length;

\}

Perform peak detections on step length, and number of peaks is the step number; Perform Right/Left foot detection (optional)

Calculate velocity as Equation (2), Step time as Equation(3), Step length as Equation (4).

\subsubsection{Capturing Vicon Gait Parameters for Validation}

The three-dimensional motion analysis system, Vicon MX, allows for very accurate measurement of movement, using reflective markers and seven cameras simultaneously. The cameras send out infrared light signals and detect the reflection from the markers attached to each subject's shoes. Based on the angle and time delay between the original and reflected signals, it tracks the movement trajectories of the reflective markers in 3D space.

The trajectory of the markers is projected onto the 2D floor, as show in Fig.3.8. The key in analyzing the Vicon data is the accurate detection of the contact of the footfall by calculating the markers location $\boldsymbol{M}(\mathrm{t})$ at time $t$. A footfall is a momentarily stationary 
location of the foot based on a threshold:

$$
|\boldsymbol{M}(t)-\boldsymbol{M}(t-1)|<\text { threshold }
$$

The threshold is selected to be $1-4 \mathrm{~mm}$ in each of the $2 \mathrm{D}$ directions depending on the person's gait style.

Once the footfalls are identified, the gait parameters can be extracted based on the GAITRite definitions given in Section3.4.1. Fig.3.8 shows an example of the foot marker trajectory, with $\mathrm{x}$ marking the left footfall locations and + marking the right footfall locations.

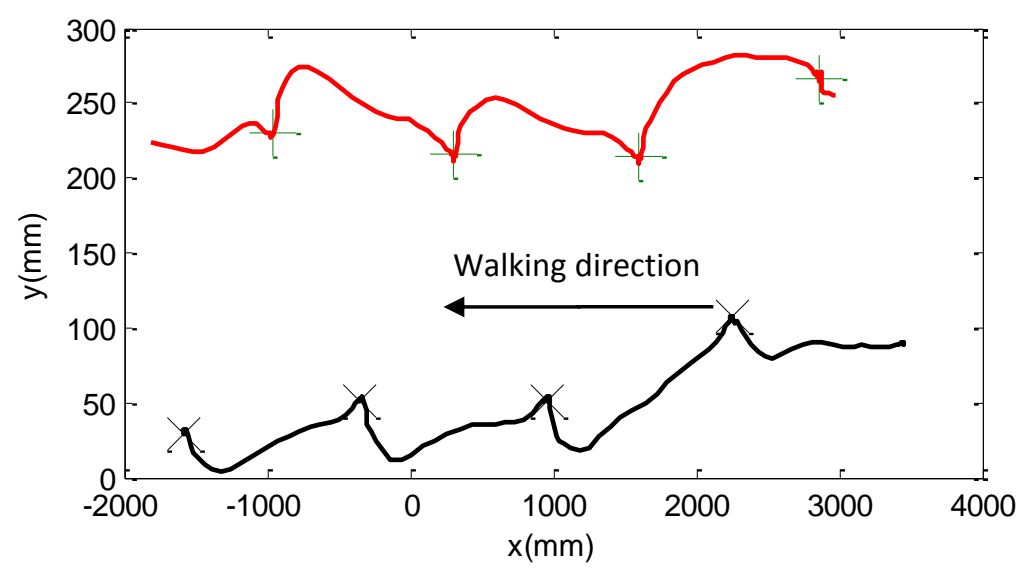

Fig.3.8 The projected 2D trajectory of the Vicon markers attached to a participant's feet, with footfall locations marked ( $x$ for left foot and + for right foot).

\subsection{Body Sway Measurement}

\subsubsection{Body Sway during Standing}

Body sway during standing includes sway in lateral and anterior-posterior directions. Body centroid $\left(x_{c t r}, y_{c t r}, z_{c t r}\right)$ was estimated using the 3-D reconstructed voxel person described in the previous section. A fixed mid-point $\left(x_{r e f}, y_{r e f}\right)$ was computed from the mean of centroid positions, and selected as a reference point on the ground plane for the 
base of support with $z_{\text {ref }}=0$. The sway distance/amplitude is computed as the distance between the body centroid projection onto the ground plane and the reference point in 2D space as:

$$
d=\sqrt{\left(x_{c t r}-x_{r e f}\right)^{2}-\left(y_{c t r}-y_{r e f}\right)^{2}}
$$

where $x$ and $y$ are the coordinate positions in the anterior-posterior (x) and lateral (y) directions (Fig.3.9c). Fig.3.10 illustrates a sway amplitude curve, where sway amplitude varies with time (frame number). As we are most interested in the maximum sway amplitude, a peak detection algorithm is applied to the curve. Usually, a curve smoothing technique is necessary prior to peak detection. Maximum sway location and frame are identified (red cross) in Fig.3.10.

In addition to the sway amplitude, this research also covers sway angle. The sway angle can be computed as the angle between two 3-D vectors, as seen in Fig.3.11(a). One vector is the axis connecting the body centroid and the base of support reference point (red line in Fig.3.9c), and the other vector is either the x-axis for anterior-posterior sway or the y-axis for lateral sway. Fig.3.11(b) illustrates the lateral sway angle computed with respect to the y-axis. 

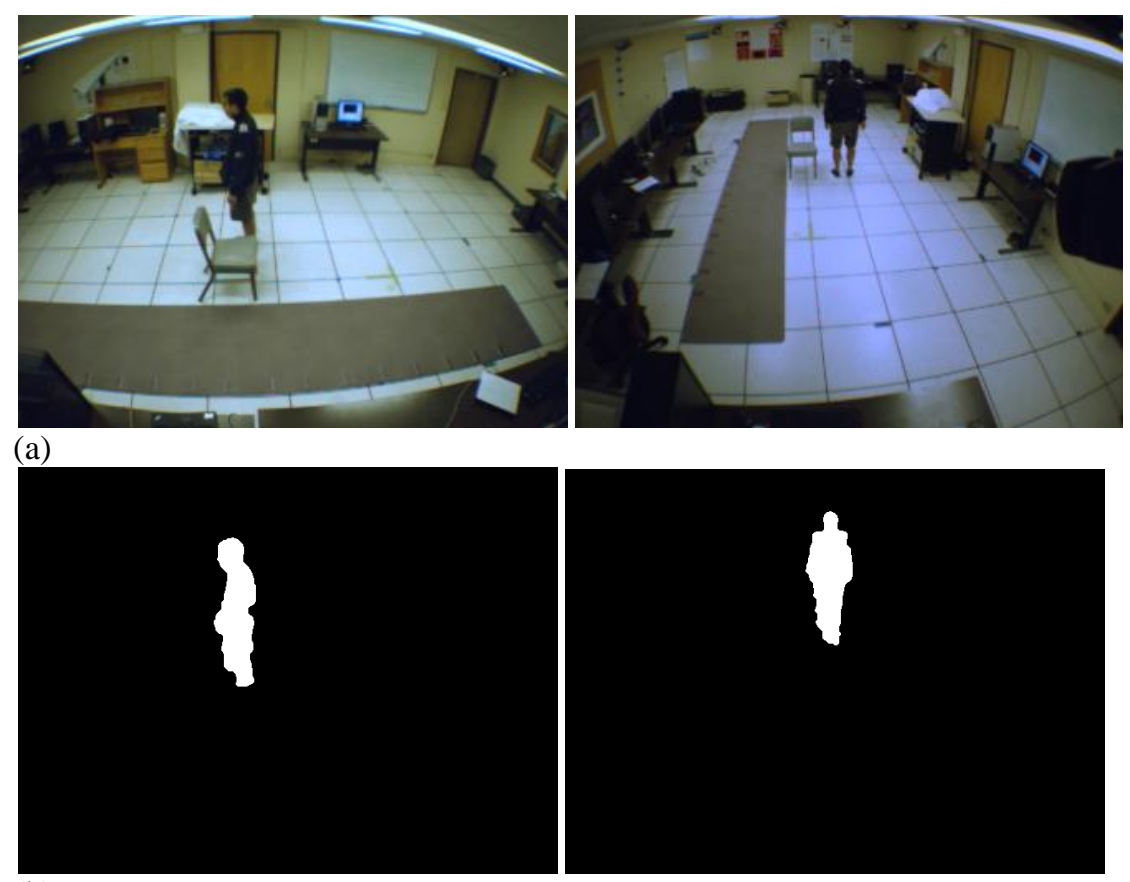

(b)

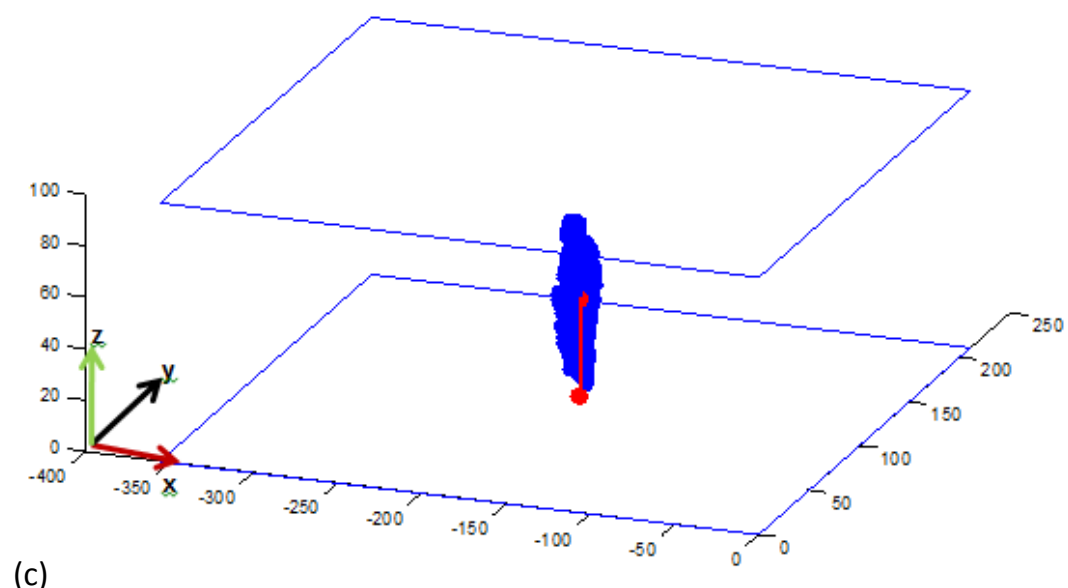

Fig.3.9 (a) Two raw camera images monitoring the same scene. (b) Human silhouettes (c) The reconstructed three-dimensional voxel person. The reference point and body centroid are denoted in red. 


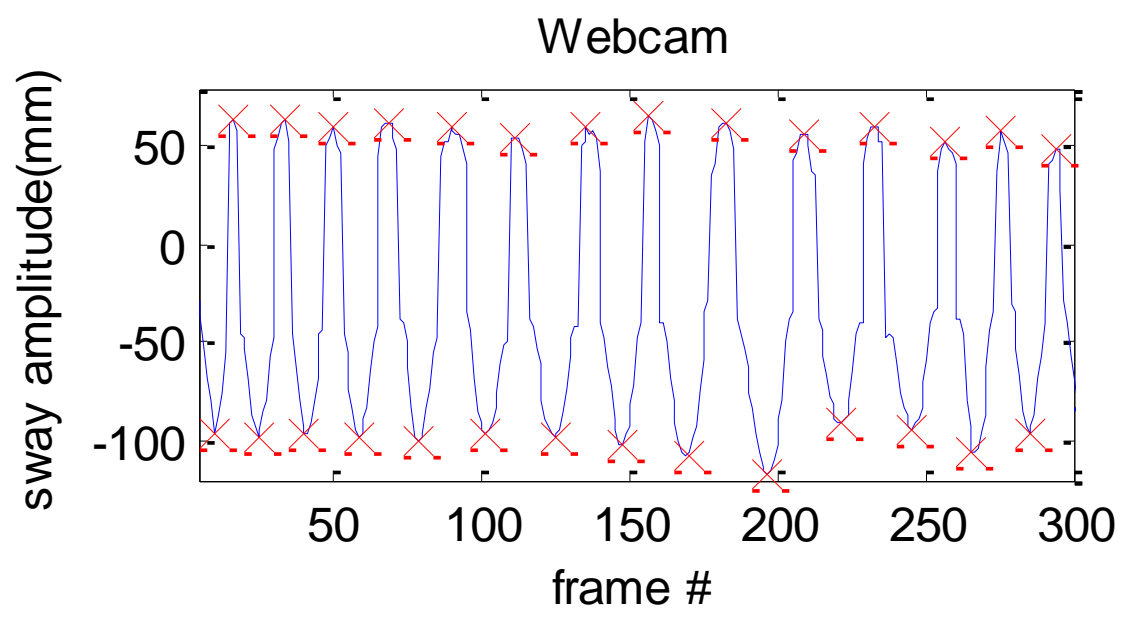

Fig.3.10 Sample sway amplitude curve during standing. Red crosses mark the maximum sway amplitude and time (frame).
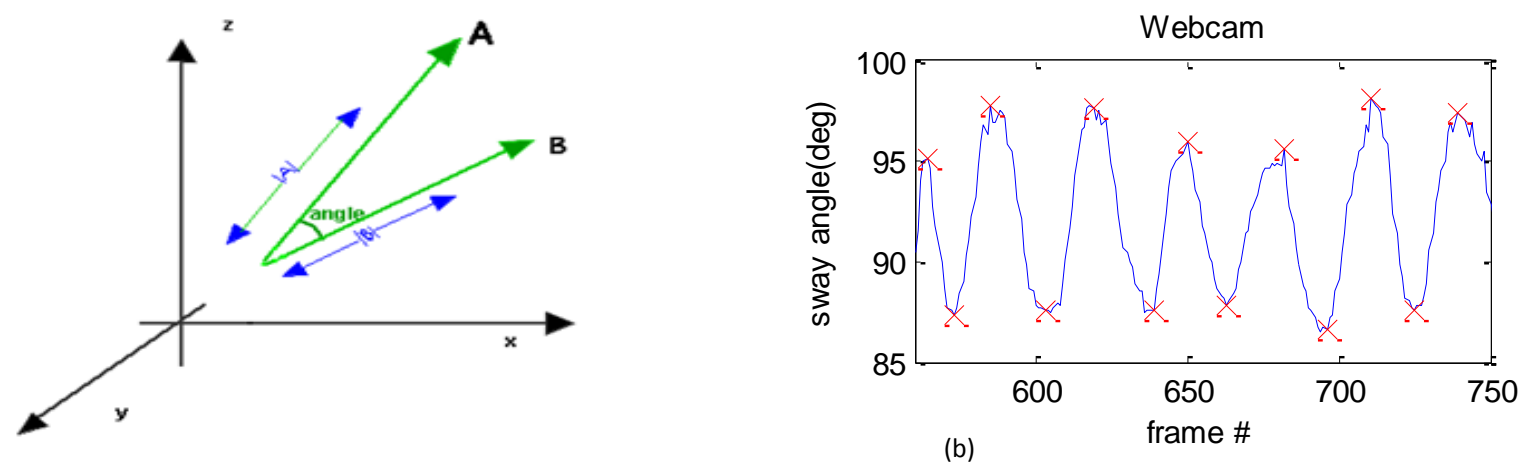

Fig.3.11 (a) Angle between 3-D vectors (b) Sample lateral sway angle during standing. Red crosses mark the maximum sway angle and time (frame).

\subsubsection{Body Sway during Walking}

Body sway analysis during walking is more complicated compared to standing. Only lateral body sway can be analyzed due to the nature of the forward motion during walking. Fig.3.12 (b) shows the trajectory of the body centroid extracted from voxel person during walking. First, peak sway centroid locations were identified, and a midpoint between the consecutive peak locations was computed. A linear interpolation is 
used to connect these mid-points and used as a line of progression. The sway amplitude is then calculated as the distance from each body centroid position to the line of progression. Fig.3.12 (a) is a sample voxel person during a walk, and (b) and (c) illustrate the 2-D body centroid trajectory and extracted sway amplitude during a sample walk.

Algorithm 4: Lateral body sway during walking

Input: Voxel $3 D$ centroid data centroid(t)

Output: Peak-peak lateral sway amplitude

Calculate the initial body centroid middle line by averaging centroid(t-2) to centroid $(t+2)$;

Project the centroid(t) onto the middle line, and calculate the distance;

Find peak points;

Recalculate the middle line with the peak points;

Re-project the centroid points on the new middle line, and calculate the distance;

Find the peak distance;

Average the peak distance; calculate the peak-peak sway amplitude;

\subsubsection{Vicon Marker-Based Motion Capture for Ground Truth}

Markers were attached to subjects' feet, shoulders and back, and their 3-D positions were recorded by the Vicon system during the experiments. Body centroid locations and trajectory can be easily obtained through the back marker positions. A similar methodology as described in the previous sections is applied to extract the body sway parameters using body centroid information. Extracted sway parameters from the Vicon system are then used for validation purpose. 

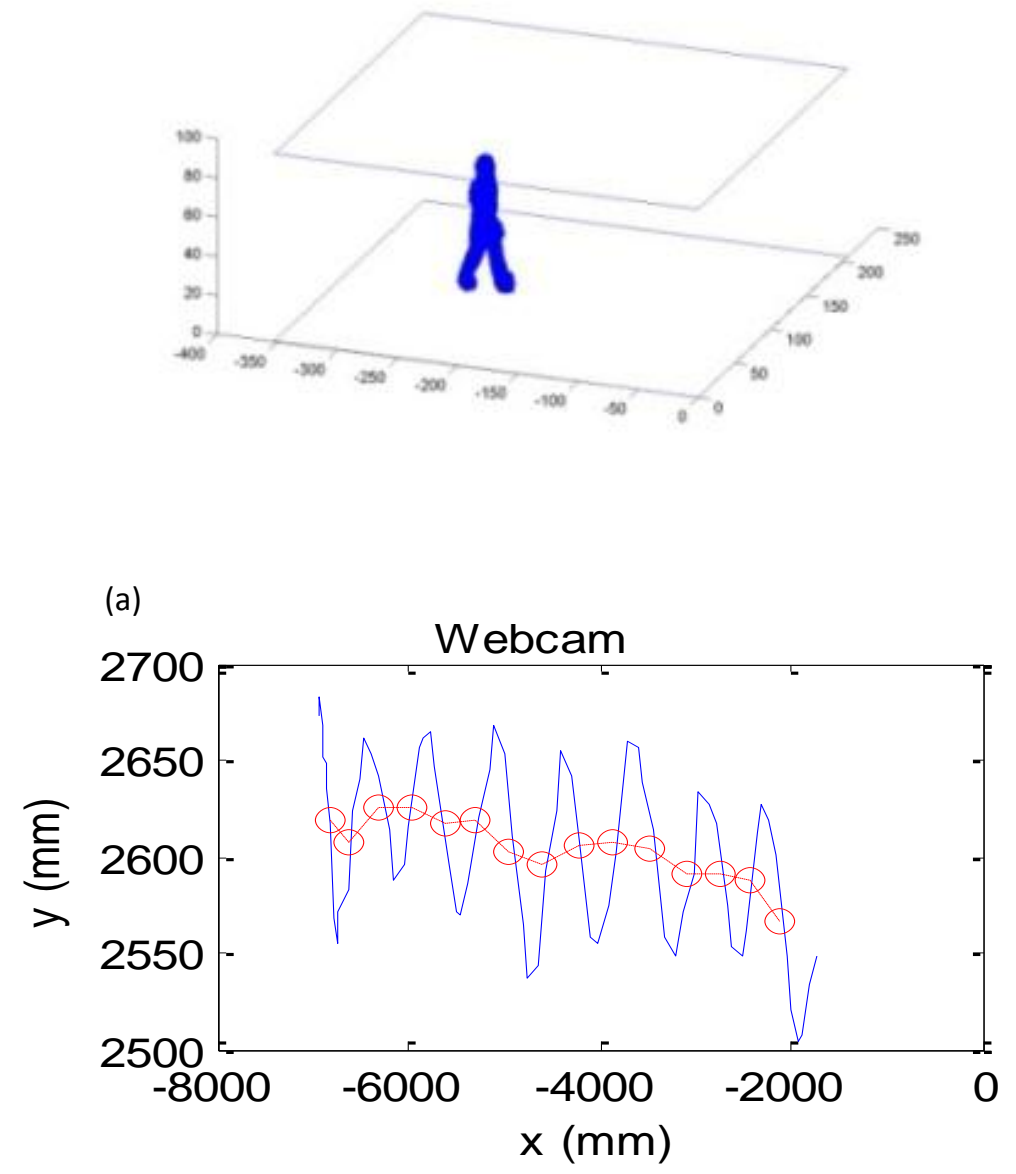

(b)

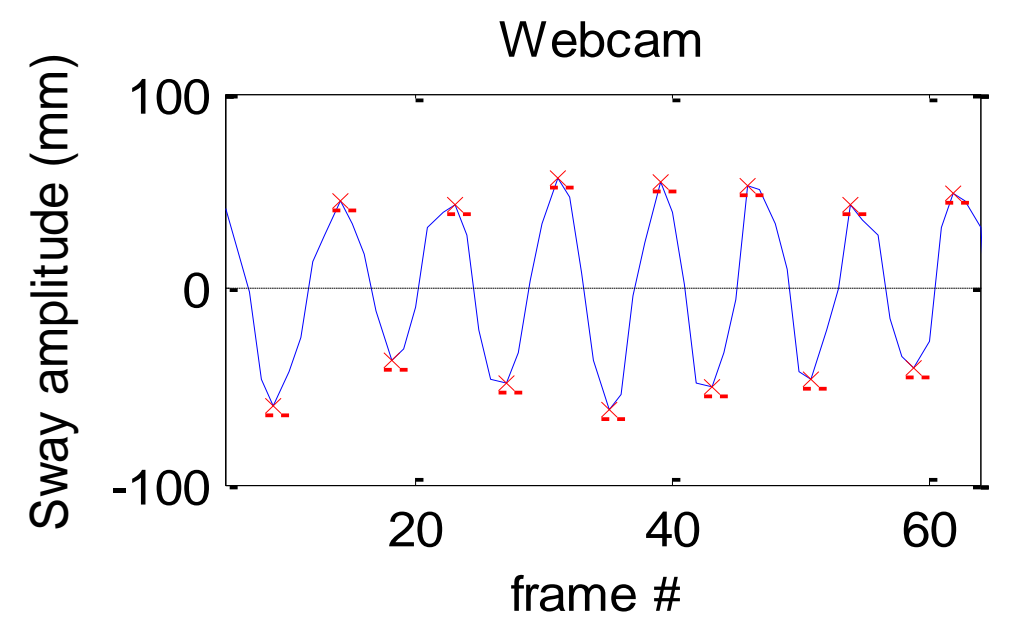

(c)

Fig.3.12 (a) Voxel person during walking (b) Body centroid trajectory during walking projected onto 2-D space. (c) Sway amplitude in med-lateral direction. Red crosses mark the maximum sway angle and time (frame). 


\subsection{Turn Analysis and Turn Recognition}

\subsubsection{Turn Time}

The body centroid for voxel person is estimated as the mean of all voxel locations detected as part of voxel person for that frame. In Fig.3.13 (a), a person's body centroid extracted from the reconstructed $3 \mathrm{D}$ voxel person is projected onto a $2 \mathrm{D}$ plane during a TUG test. The circled region is where the 180 degree turn occurs. The velocity vector $\boldsymbol{V}$ is computed using the centroid locations from the two consecutive frames as illustrated in Fig. 2(b). The angle is then calculated between two consecutive velocity vectors as:

$$
\theta=\cos ^{-1}\left(\frac{V_{1} \cdot V_{2}}{\left|V_{1}\right|\left|V_{2}\right|}\right)
$$

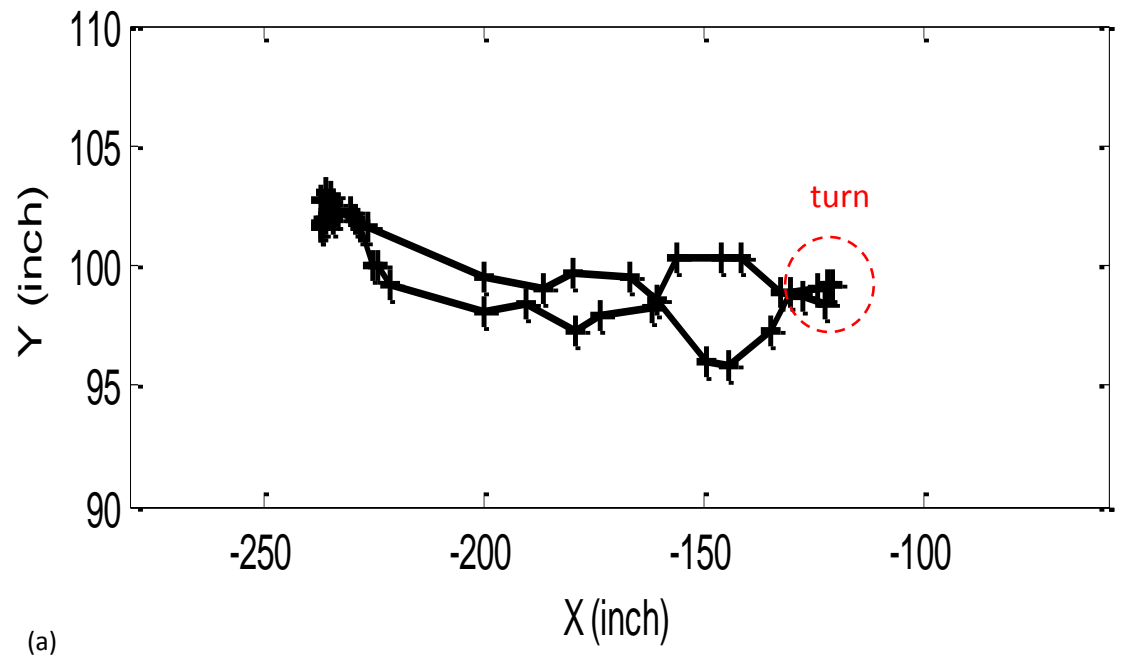

(a)

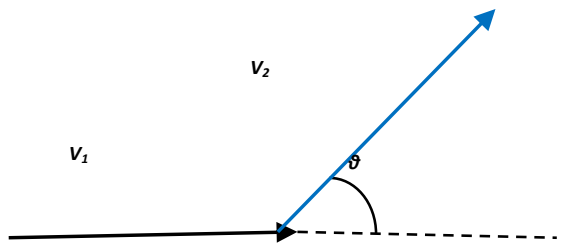

(b)

Fig.3.13 (a) A person's walk and turn trajectory during the TUG test. (b) Velocity vector illustration 
The turn starts when the voxel person trajectory begins to deviate from the straight walk, and ends when it returns back to the straight walk. The calculated angles between velocity vectors are shown in Fig. 3. It clearly shows a large angle change where the 180 degree turn takes place.

The extracted angle data is then fitted with a Gaussian model described in Eq. (8).

$$
y=y_{0}+A * \exp \left(-\frac{\left(x-x_{c}\right)^{2}}{2 w^{2}}\right)
$$

where $y_{0}$ represents the offset angle values, $A$ is the peak angle amplitude; $x_{c}$ is the center of the peak; and $w$ represents the standard deviation. The turn time is defined using the 3sigma rule, which is $6^{*} w$.

(a)

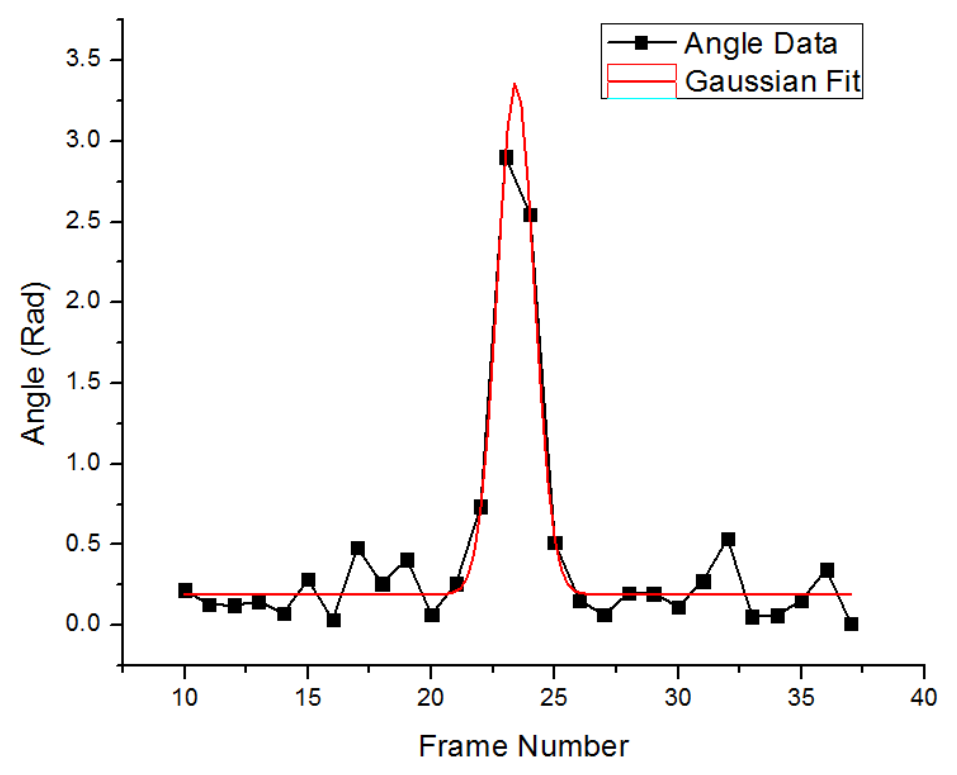




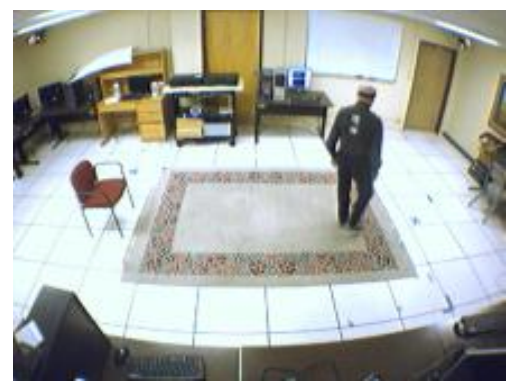

21

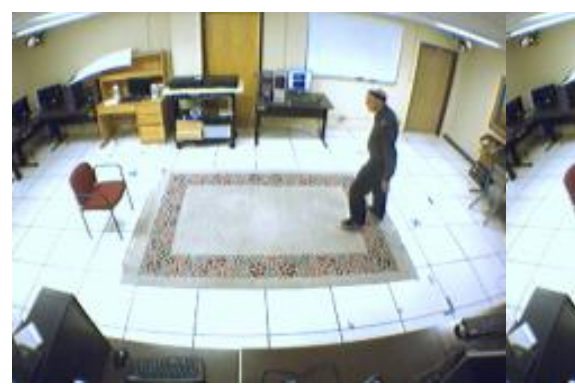

(b)

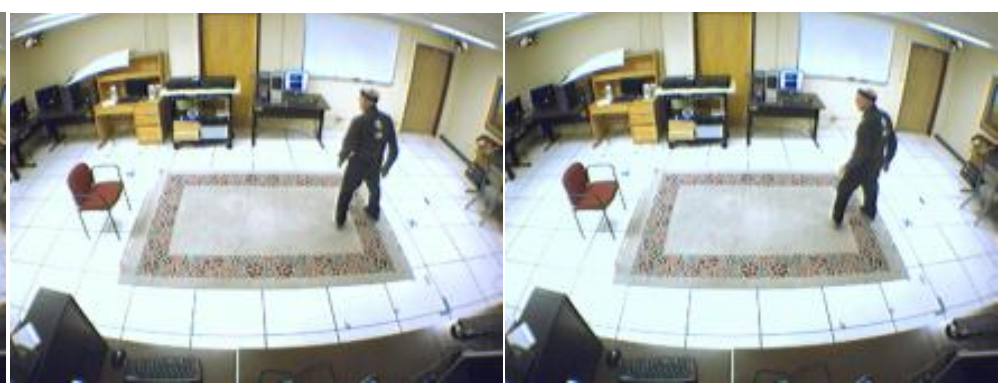

22

23

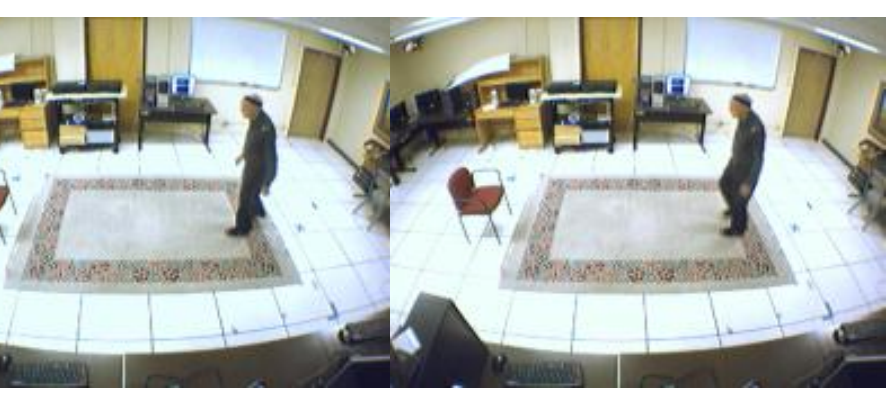

25
26

Fig.3.14 (a) Angles extracted from velocity vectors. (b) Image 21-26 from one camera view detected as a turn using the model.

\subsubsection{Number of Turn Steps}

To count the number of steps taken in the turn, F. Wang et al. use a method they previously developed to detect steps during walking [54]. In this method, the voxels with a height below 4 inches from the ground plane are used to capture foot motion. They are projected onto the $2 \mathrm{D}$ space, and the length from the front of one foot to the end of the other foot projected along the walking direction alternatively expands (shown as peaks) and contracts (shown as valleys) over time as the person's feet spread and close during the gait cycle (Fig.3.15). The number of steps is obtained directly from the number of peaks representing the number of gait cycles. In Fig. 4, a turn is detected from frame \#23 to \#30, where frame \#25 and \#29 have peak step length; the corresponding images are shown in Fig.3.15 (b). 
(a)

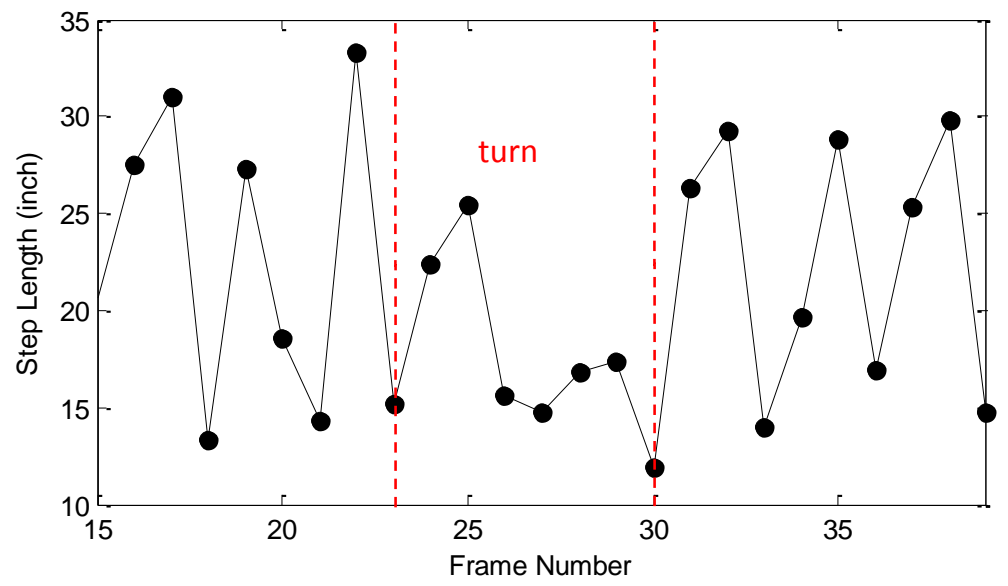

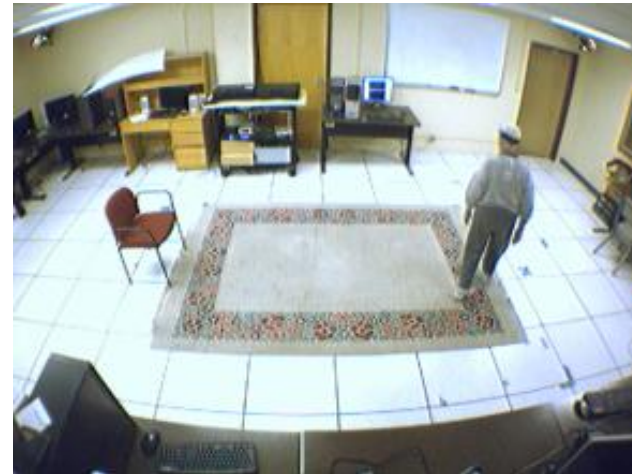

(b) Frame 25

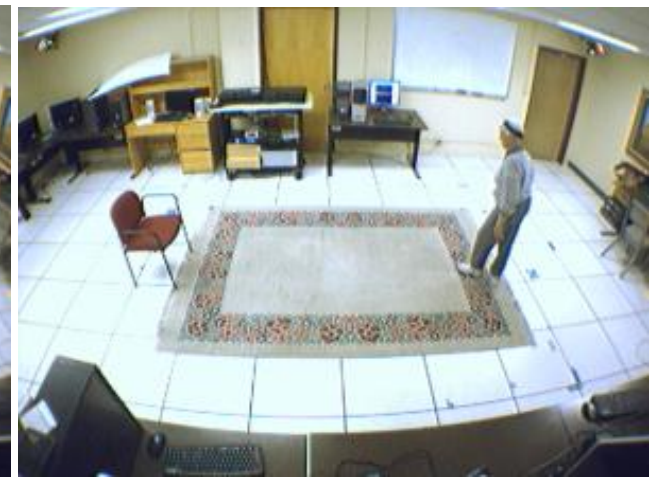

(c) Frame 29

Fig.3.15 (a) Step length variation during the walk and turn. (b) Images $25 \& 29$ from one camera view which are detected as peak step lengths during a turn.

\subsubsection{Turn Recognition}

Another research priority for this dissertation was finding turn around frames for the study of turns. Velocity vector angles, as introduced in section 3.6, are extracted and used for this purpose. Fig.4.11 illustrates an example velocity vector angles for ID4 test 2. A frame will be selected for a possible turn around frame if it satisfies the following conditions:

(1) Its velocity vector value is a local peak

(2) Its velocity vector value is above threshold value of 0.9 . 
(3) It does not fall in the idle state where the person has a very low speed for an extended time period.

For ID4 Test 2, the algorithm has identified the following frames as the potential turn around frames: 1311, 1357, 1392, 1404, 1424, 1643, 1658, 1704, and 1824. They are all verified to be turn around relevant frames. Some of the examples are illustrated in Fig.3.17.
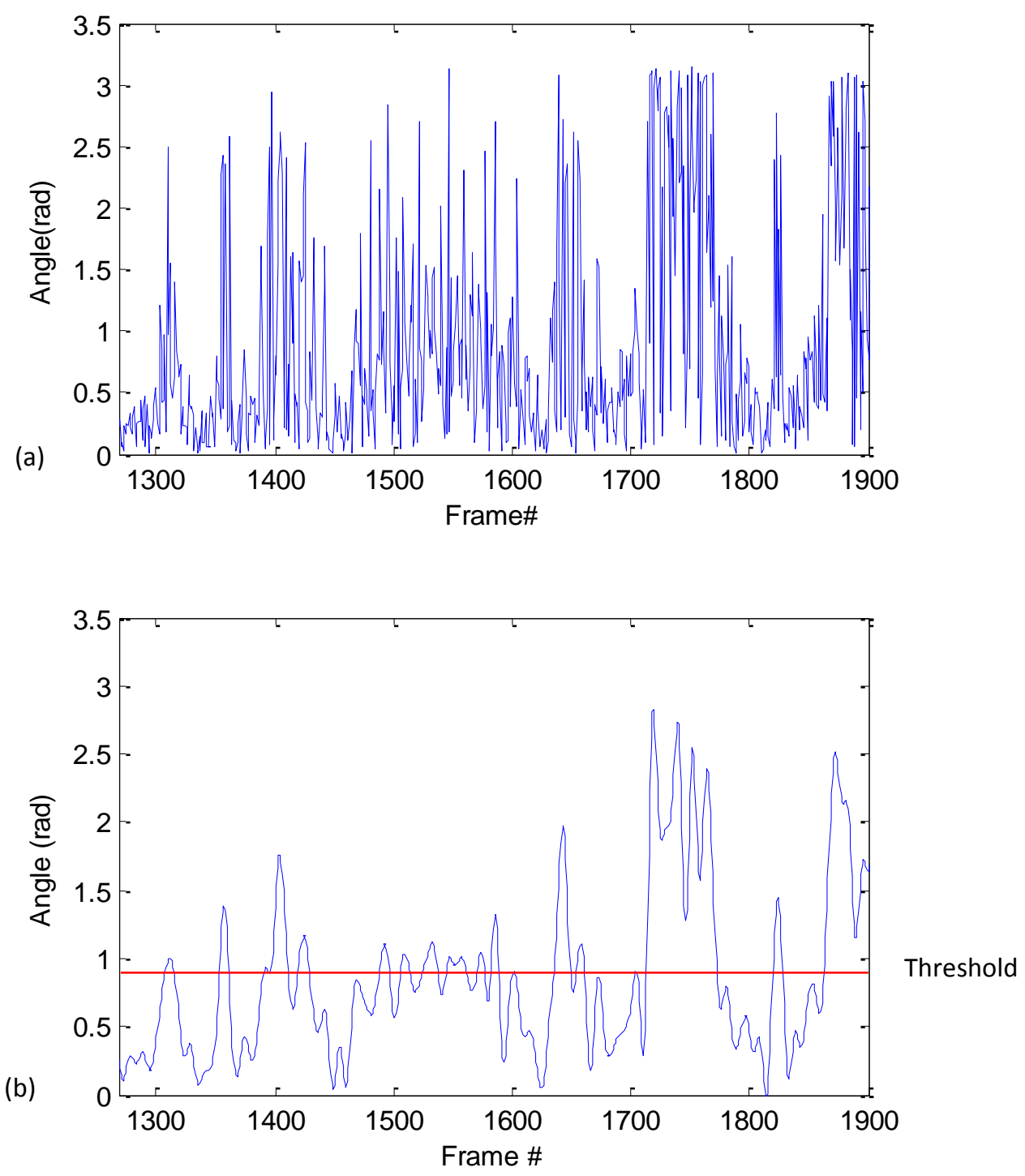

Fig.3.16 ID4 Test 2 velocity overview (a) velocity vector angles (b) cubic spline smoothing velocity angels used to segment turn around 

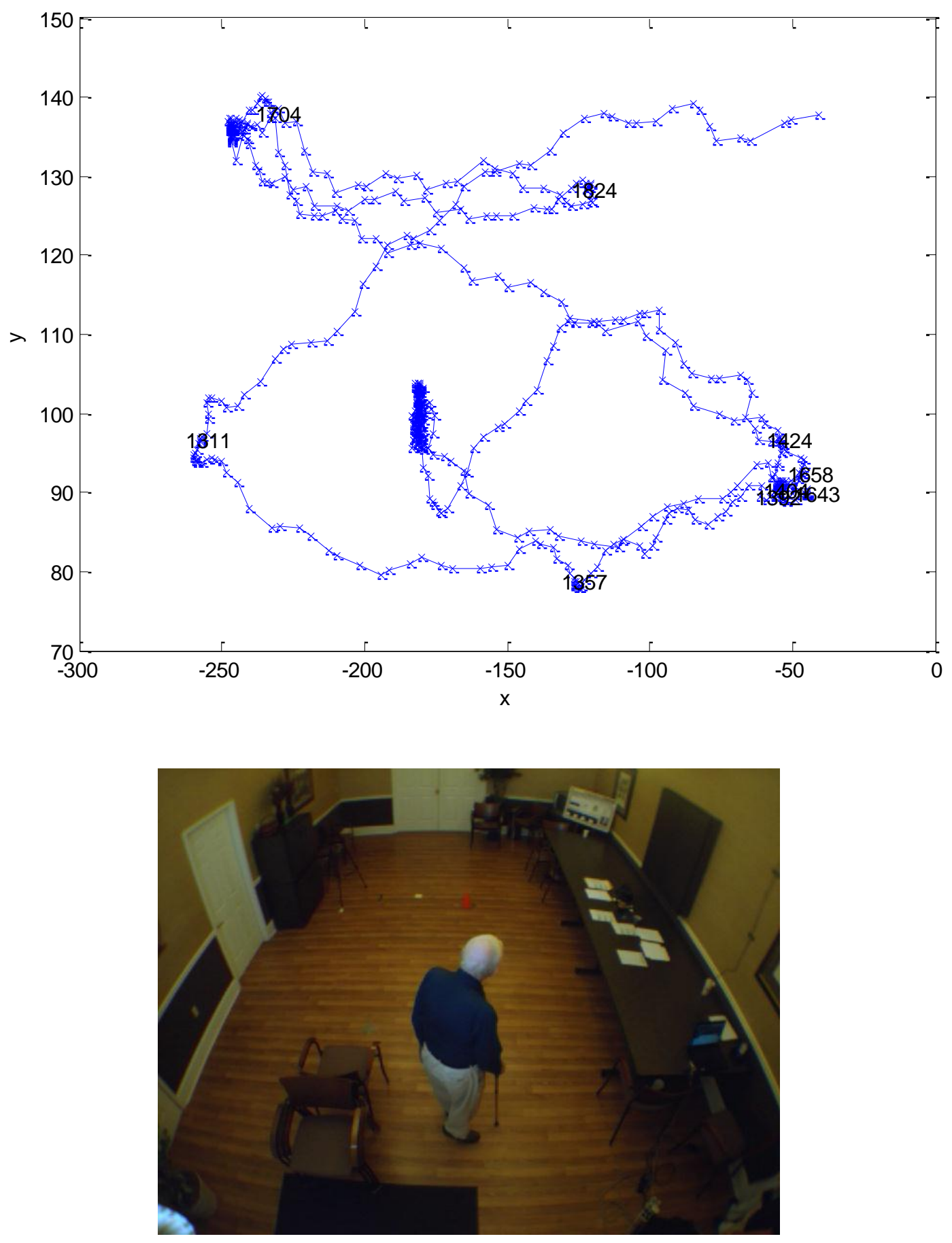

Frame 1311 


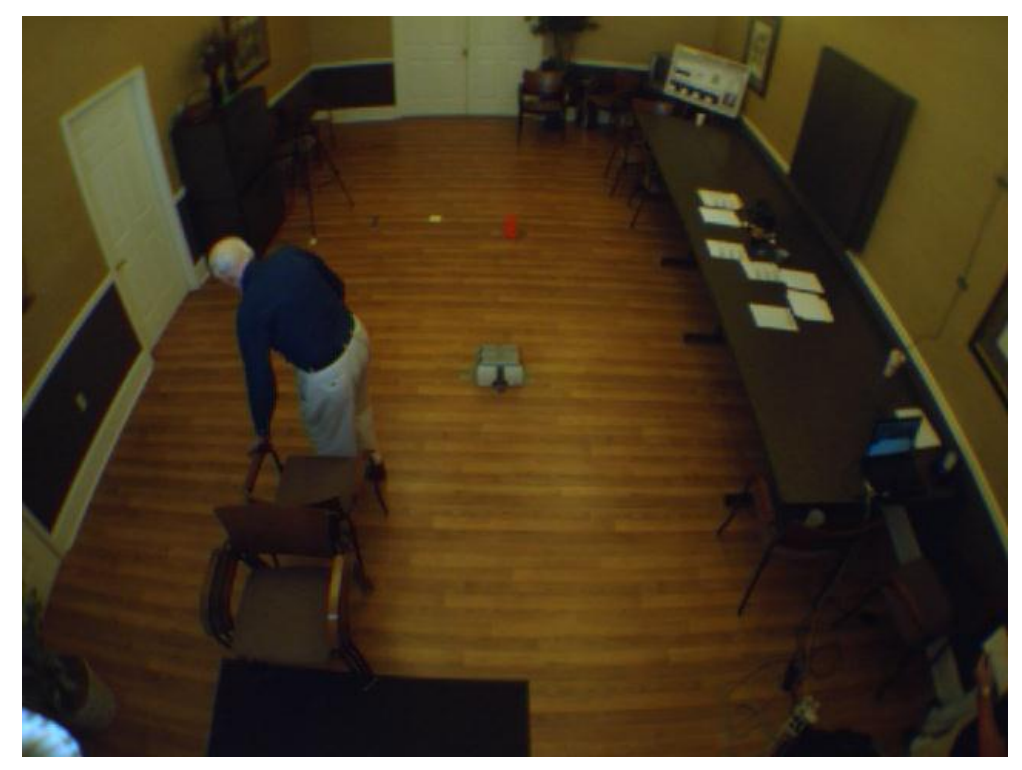

Frame 1704

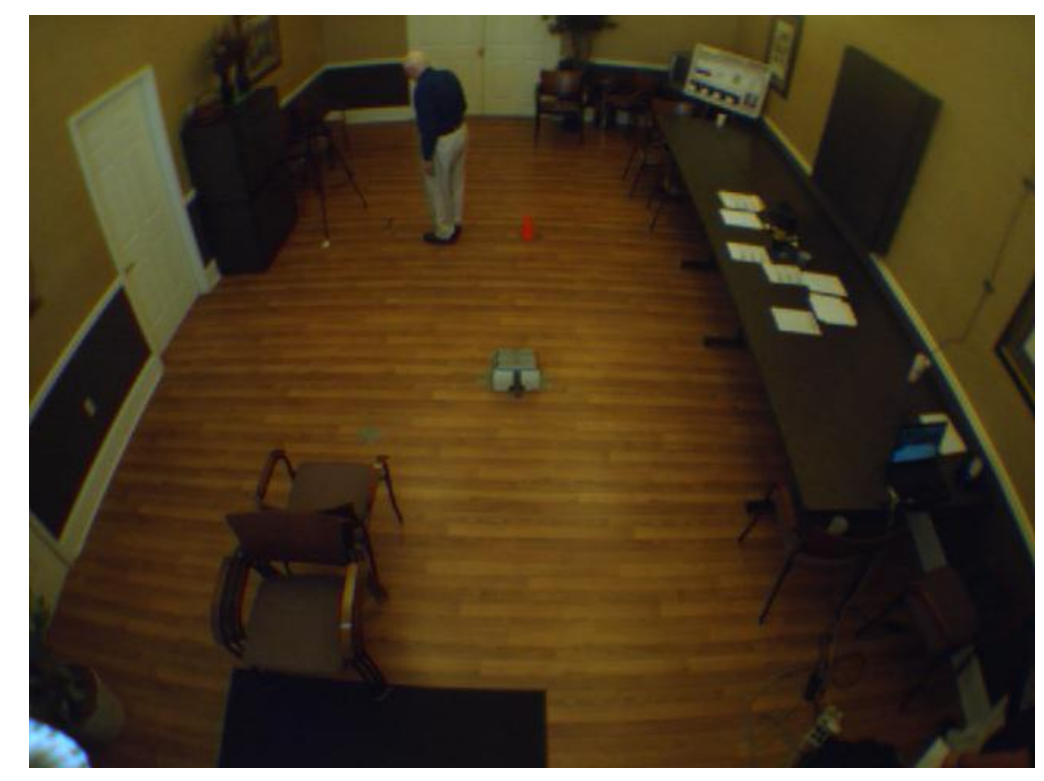

Frame 1824

Fig.3.17 ID4 Test 2 turn around (a) voxel person trajectory projected on ground plane with turn around frames marked (b) turn around images illustration: frame 1311, 1704, and 1824. 
Algorithm 5: Turn recognition

Input: Voxel 3D centroid data, threshold for velocity $T_{v}$, threshold for voxel foot area $T_{f}$, threshold for number of consecutive frames $T_{i}$; threshold for velocity vector angles $T_{a}$;

Output: Turn frames

Calculate velocity, and apply curve smoothing;

Calculate velocity vector angle as Equation (7), and apply curve smoothing;

Eliminate bad frames that voxel lower than 5 "'(foot area)below threshold $T_{f}$;

Select frames below threshold velocity $T_{v}$;

Select idle frames above $T_{i}$;

Select turn frames that satisfy:

(1) Corresponding velocity angle is a local peak

(2) Corresponding velocity angle is above threshold value $T_{a}$.

(3) Frame does not fall in the idle state where the person has a very low speed extended time period. 


\section{Chapter 4. Experimental Results}

\subsection{Validation Experiment for Gait Analysis}

\subsubsection{Experimental Setup}

Two stationary cameras were placed in approximately orthogonal locations to record images while the subjects walked across the GAITRite mat. For the experiments, we used Unibrain Fire-i Digital Cameras. The images are recorded at a frame rate of 5 frames per second, with an image resolution of 640x480 pixels.

Thirteen subjects participated in the test. Each subject was tested multiple times for accuracy, as well as various walking patterns, including normal speed, fast, slow, and limping. In total, there are 44 tests. Due to the camera viewing angle limitation, at the camera boundary regions where the subject enters or leaves the scene, the voxel person models are noisy. These frames are eliminated from the data analysis to reduce the errors. Subjects normally take about 8-10 steps to complete the walkway. But when different walking patterns are utilized, such as fast and slow walks, the steps could range from 6 to 16.

\subsubsection{Results}

The test results shown in Fig.4.1, and Table 1-3 list the comparison differences between the web camera, GAITRite and Vicon systems for walking speed, step time, step length respectively.

The walking speeds obtained from the tests cover a large variation ranging from around $40 \mathrm{~cm} / \mathrm{s}$ to $180 \mathrm{~cm} / \mathrm{s}$. An excellent agreement among the three systems is achieved. The standard deviation (as seen in Table 1) is around 2, which implies that most of the difference would fall within $+/-6 \%$ (3sigma) when comparing the systems to each other. 
As for the test with the largest difference, the difference of the web camera vs. the GAITRite is $7.9 \%$, and the Vicon vs. the GAITRite is $6.4 \%$. However, the web camera was good match for the Vicon with a difference of $1.4 \%$. One reason that might cause the larger difference is the very low walking speed for this particular test (GAITRite 32.9 $\mathrm{cm} / \mathrm{s}$, Vicon $35.0 \mathrm{~cm} / \mathrm{s}$, and web camera $35.5 \mathrm{~cm} / \mathrm{s}$ ). Although the absolute difference in walking speed is small, the percentage difference is quite large.

Table 2 lists the results for step time. The camera frame rate (5fps) needs to be considered when discussing step time. Based on Nyquist-Shannon's theorem, the Nyquist sampling rate needs to be twice the highest signal frequency, which would translate to 0.4s step time for the TigerPlace camera system. This research's results have shown all the subjects' step time is longer than $0.4 \mathrm{~s}$ (with subject age ranging from early 20 's to early 60's). Considering the technology application for elderly people, the current frame rate is adequate to capture the step time accurately. It has been observed that the tests with large differences are the ones with a short step time, fast walking speed, and large step length. In the tests with the largest difference shown in Table 2, the subject takes only 6 steps compared to the $8-10$ steps normally needed to complete the walkway. In this case, for the same walkway length, there are fewer steps available to analyze. The step time obtained for this test from the GAITRite is $0.43 \mathrm{~s}, 0.44 \mathrm{~s}$ for Vicon, and 0.40 s for the web cameras. The average step time for all subjects tested is around 0.6s. So the rational conclusion is that the large difference is due to a fewer number of steps as well as a shorter step time (fewer steps).

The average step length for GAITRite is obtained through the average of the step length reading of the right and left feet. And the average step length for both the Vicon 
and the web camera system is calculated using walking speed multiplied times the step time. Therefore, the average step length depends on the two gait parameters discussed earlier. It is not surprising to find that the step length difference is very similar to the step time difference, which implies that the difference in step length mainly is caused by step time difference among the three systems compared.

Overall, all the gait parameters extracted from the web camera system showed good agreement compared to GAITRite and Vicon. The largest difference occurs only at extreme conditions, such as very low walking speed, or an extremely fast step cycle. Still for the very low walking speed, the web camera and Vicon match well. As for the errors caused by short step time, it can be resolved by having a longer walk distance. Here, it is not a big concern as we are focusing on an elderly population.

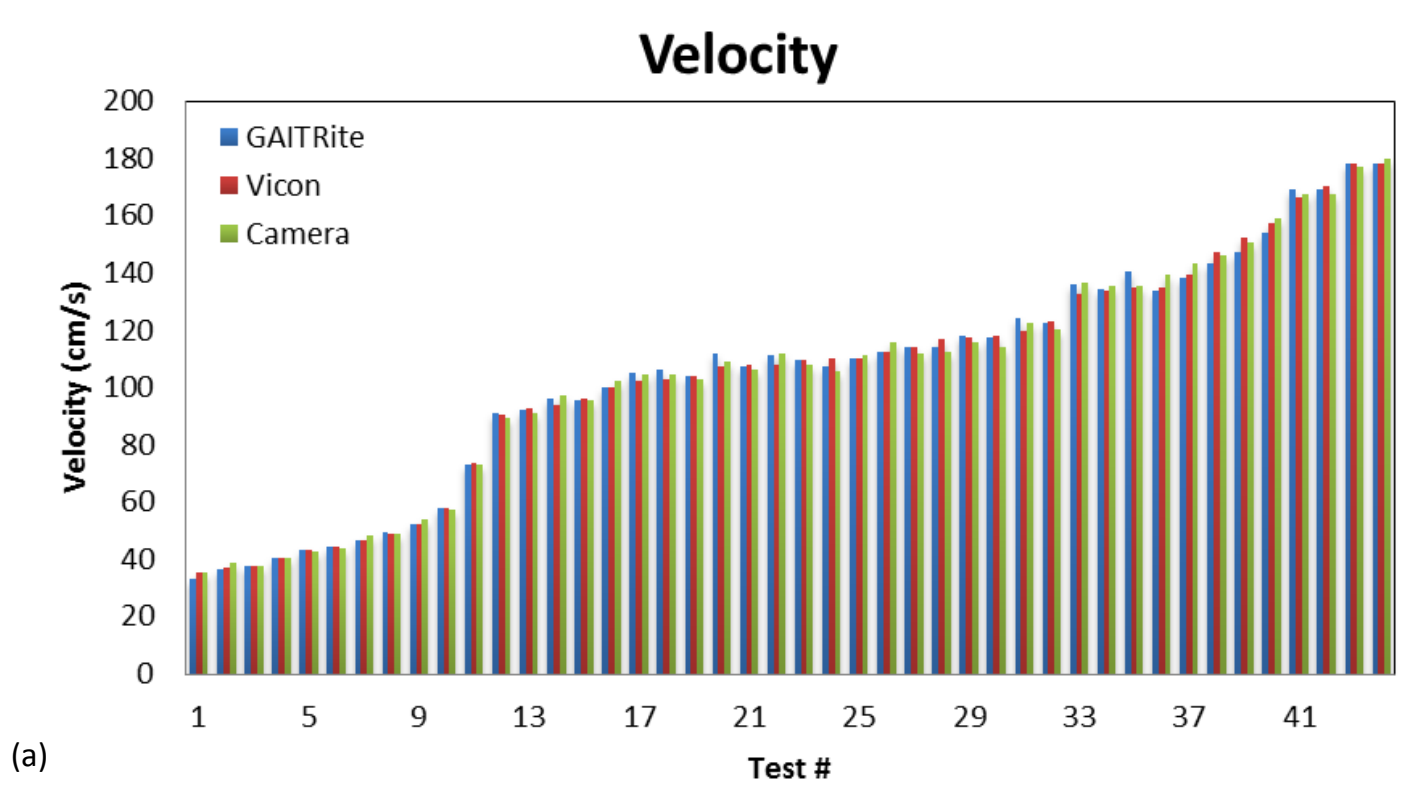




\section{Step Time}
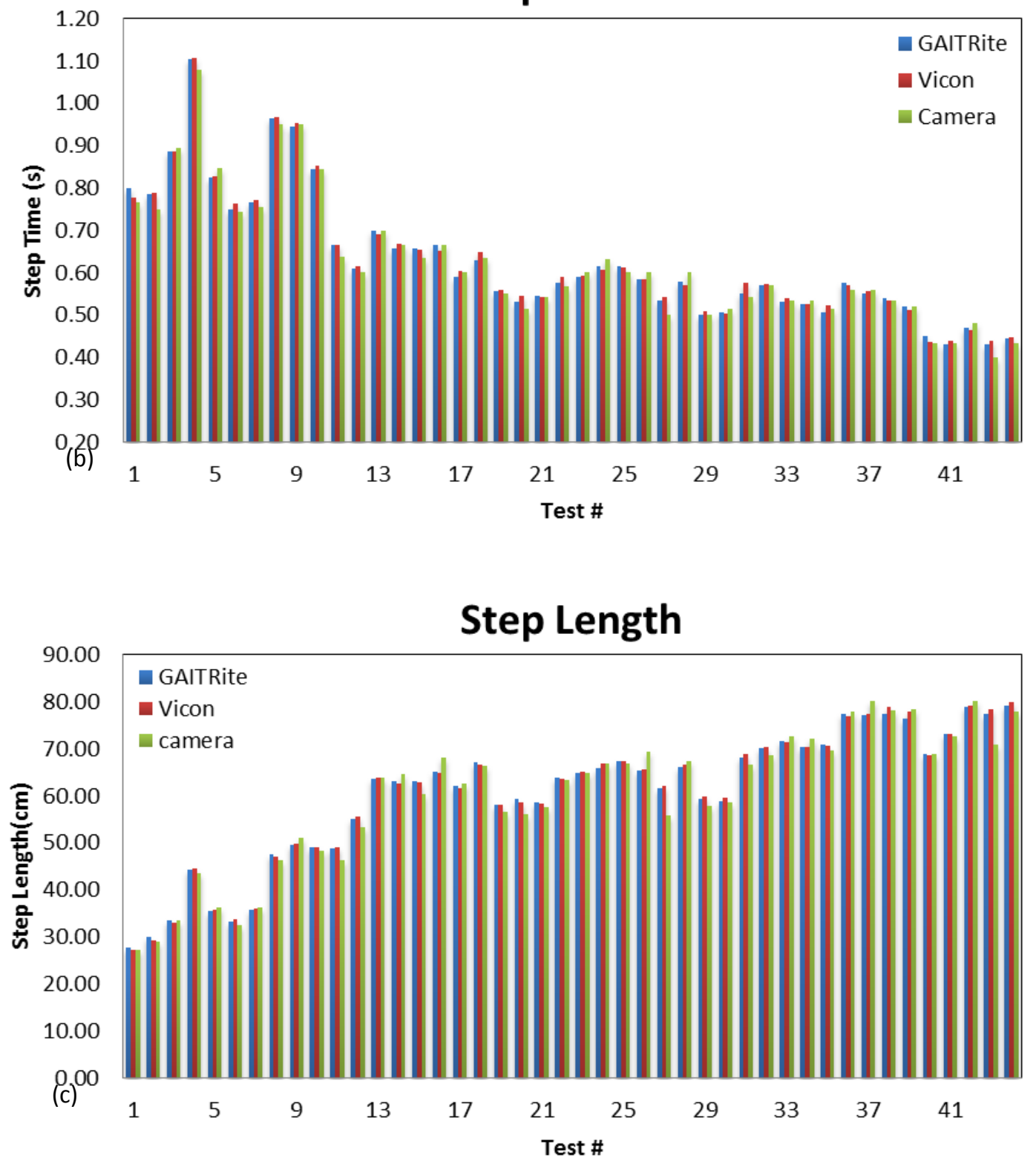

Fig.4.1(a)Velocity (b) Step Time (c) Step Length results for camera, GAITRite and Vicon systems 
Table 1. Walking Speed: Camera vs. GAITRite and Vicon

\begin{tabular}{|l|c|c|c|}
\hline & avg diff $(\%)$ & $\max \operatorname{diff}(\%)$ & stdev \\
\hline Camera vs. GAITRite & 0.18 & 7.92 & 2.32 \\
\hline Camera vs. Vicon & 0.29 & -3.91 & 2.07 \\
\hline Vicon vs. GAITRite & -0.09 & 6.45 & 1.99 \\
\hline
\end{tabular}

Table 2. Step Time: Camera vs. GAITRite and Vicon

\begin{tabular}{|l|c|c|c|}
\hline & avg diff $(\%)$ & $\max \operatorname{diff}(\%)$ & stdev \\
\hline Camera vs. GAITRite & -0.63 & -6.98 & 2.46 \\
\hline Camera vs. Vicon & -1.08 & -9.09 & 2.95 \\
\hline Vicon vs. GAITRite & 0.48 & 4.85 & 1.65 \\
\hline
\end{tabular}

Table 3. Step Length: Camera vs. GAITRite and Vicon

\begin{tabular}{|l|c|c|c|}
\hline & avg diff $(\%)$ & $\max \operatorname{diff}(\%)$ & stdev \\
\hline Camera vs. GAITRite & -0.71 & -9.47 & 3.04 \\
\hline Camera vs. Vicon & -0.81 & -9.91 & 3.10 \\
\hline Vicon vs. GAITRite & 0.10 & -2.27 & 0.88 \\
\hline
\end{tabular}

In order to further understand how well the systems match to each other, a t-test is performed to see if the differences among the systems are statistically significant. Table 4 lists the $p$ value results. It has been observed that $p<0.02$ for the step time camera vs. Vicon, and GAITRite vs. Vicon, which means that the difference is statistically significant. However, $p>0.05$ for the camera vs. GAITRite, which means the difference observed between the two is not significant. This interesting observation could be a result of how the footfalls were extracted for the Vicon. Since the camera results have no significant difference from the GAITRite results, we are still confident with the step time 
results from the camera. In addition, $p>0.05$ for all walking speed and step length comparisons, meaning the difference is not significant.

Table 4. Statistical Test (T-test) for camera, GAITRite and Vicon results

\begin{tabular}{|l|c|c|c|}
\hline \multicolumn{1}{|c|}{$p$ value } & Walking Speed & Step Time & Step Length \\
\hline Camera vs. GAITRite & 0.59 & 0.32 & 0.32 \\
\hline Camera vs. Vicon & 0.55 & 0.01 & 0.15 \\
\hline Vicon vs. GAITRite & 0.20 & 0.01 & 0.08 \\
\hline
\end{tabular}

Table 5 shows the test results for the asymmetry in step length. It's not surprising to see that as the difference in step length asymmetry becomes smaller, the camera results become less accurate due to the voxel space resolution.

Table 5. Right/Left Step Length Difference

\begin{tabular}{|c|c|c|c|}
\hline Test \# & GAITRite $(\mathrm{cm})$ & Vicon $(\mathrm{cm})$ & Camera $(\mathrm{cm})$ \\
\hline 1 & 7.06 & 6.56 & 6.51 \\
\hline 2 & 8.13 & 8.44 & 8.82 \\
\hline 3 & 4.01 & 4.62 & 2.80 \\
\hline 4 & 4.75 & 4.05 & 4.53 \\
\hline
\end{tabular}

In addition, one webcam vs. Vicon camera comparison for a random walk test was performed specifically to verify the performance of the system while the person is walking in various directions with respect to the camera locations and in a non-straight, non-controlled walk path. In this test, a participant is asked to wander around in a $10 \times 8$ $\mathrm{ft}$ area. The participant walked 73 feet distance (22m). Comparison was made between webcam and Vicon because of the random walking nature. The average velocity from the webcam is $77.4 \mathrm{~cm} / \mathrm{s}$ and Vicon $77.6 \mathrm{~cm} / \mathrm{s}$ verifying that the system is well suited for an 
in-home environment where the person's walk is uncontrolled as compared to controlled lab settings.

\subsection{Validation Experiments for Body Sway}

\subsubsection{Sway during Standing}

The body sway test results during standing are shown in Fig.4.2. Table $6 \&$ Table 7 list the comparison differences between the webcam and Vicon systems.

The sway amplitude with respect to the mid fixed reference point is expressed in $\mathrm{mm}$ and shown in Fig.4.2(a), where red crosses mark the peak amplitude. The most significant point of interest is the maximum sway amplitude during the sway process. The average sway amplitude (peak-peak) for anterior-posterior sway is $140.4 \mathrm{~mm}$ for the webcams vs. $154.1 \mathrm{~mm}$ for the Vicon system. Looking at each individual sway cycle, the average difference between the two systems is $8.4 \%$, with a standard deviation of 9.9 . Similarly, for lateral sway, the average sway amplitude (peak-peak) is $140.4 \mathrm{~mm}$ obtained from the webcams vs. $138.0 \mathrm{~mm}$ from the Vicon system. The average difference between the two systems for each sway cycle is $3.1 \%$ with a standard deviation of 5.1. Given the voxel resolution used in this experiment is $25.4 \mathrm{~mm}$ ( 1 inch), the results extracted from the webcam voxel person matches with the Vicon results very well. Fig.4.2 (a) also shows that the sway amplitude profile and frequency obtained from the web cameras closely follow those from the Vicon system. It clearly shows that there are 35 sway cycles in the anterior-posterior direction, and 30 cycles in the lateral direction. The reason that the anterior-posterior sway has a larger difference compared to the Vicon results than the lateral sway is believed to be related to the locations of the webcams with 
respect to the subject's location, which produces a larger reconstruction error in the anterior-posterior direction at the back of the person than the lateral direction. Voxel person reconstruction using fuzzy logic has been reported to reduce such reconstruction errors [57], and could be used for further improvement.

The sway angle results are shown in Fig.4.2 (b), (c), and Table 7. As expected, the sway angles vs. $\mathrm{x}$-axis (shown in Fig.4.2b) for anterior-posterior sway shows clear oscillation signals around 90 degrees, and an almost constant signal for lateral sway. Similarly, the sway angles vs. y-axis (shown in Fig.4.2c) for lateral sway shows clear oscillation signals around 90 degrees, and an almost constant signal for anterior-posterior sway. The average peak-peak sway angle computed from voxel person is 7.6 degrees in the anterior-posterior direction, and 7.0 degrees in the lateral direction, compared to 6.6 degrees and 6.3 degrees from the Vicon system, respectively.

(a)

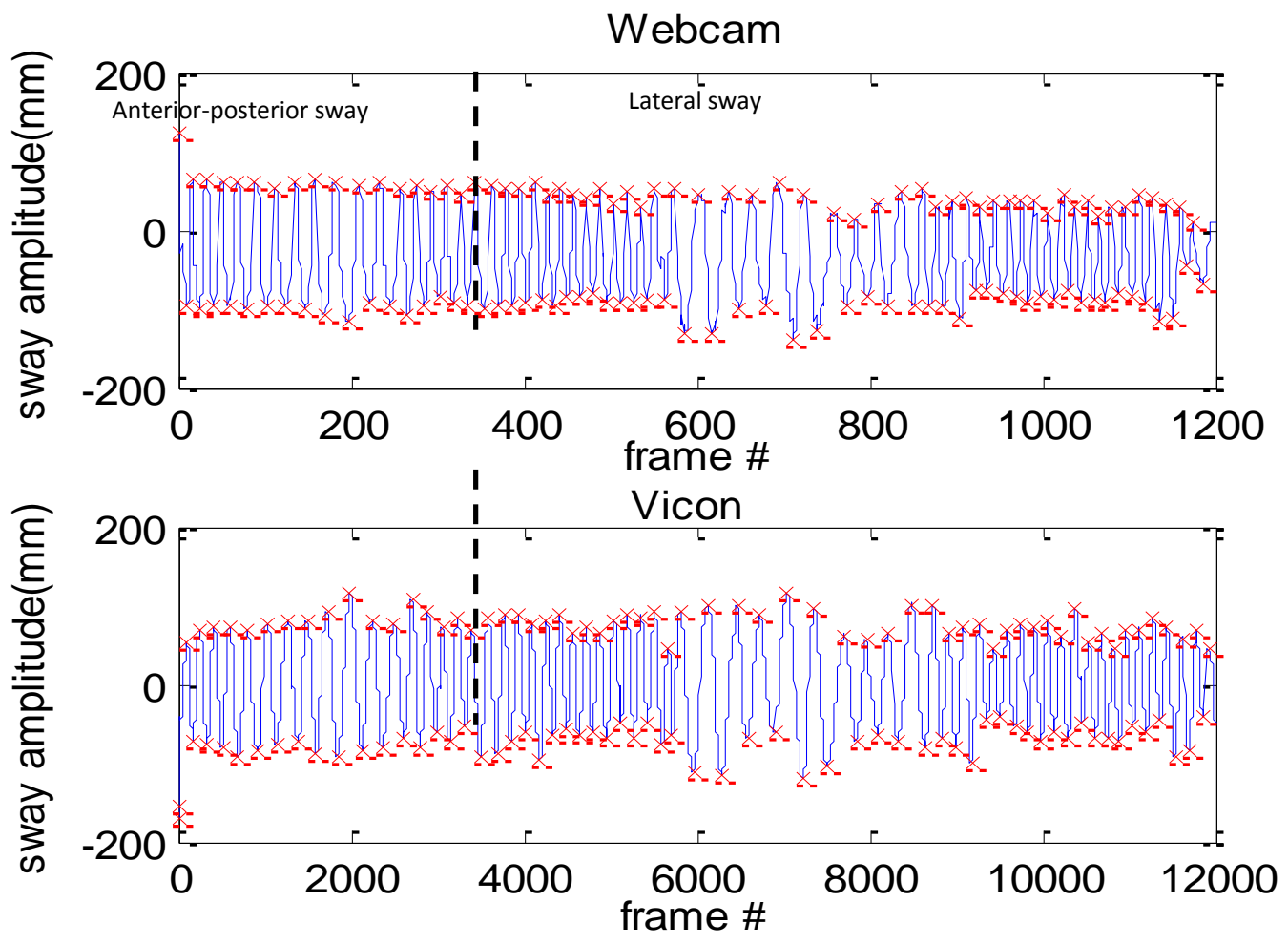




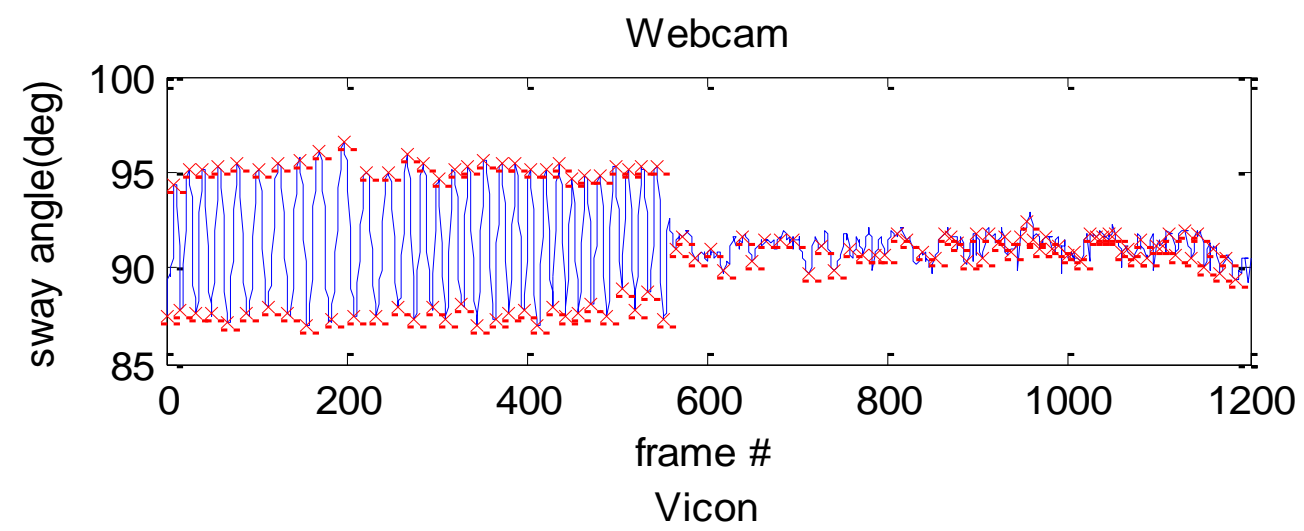

(b)
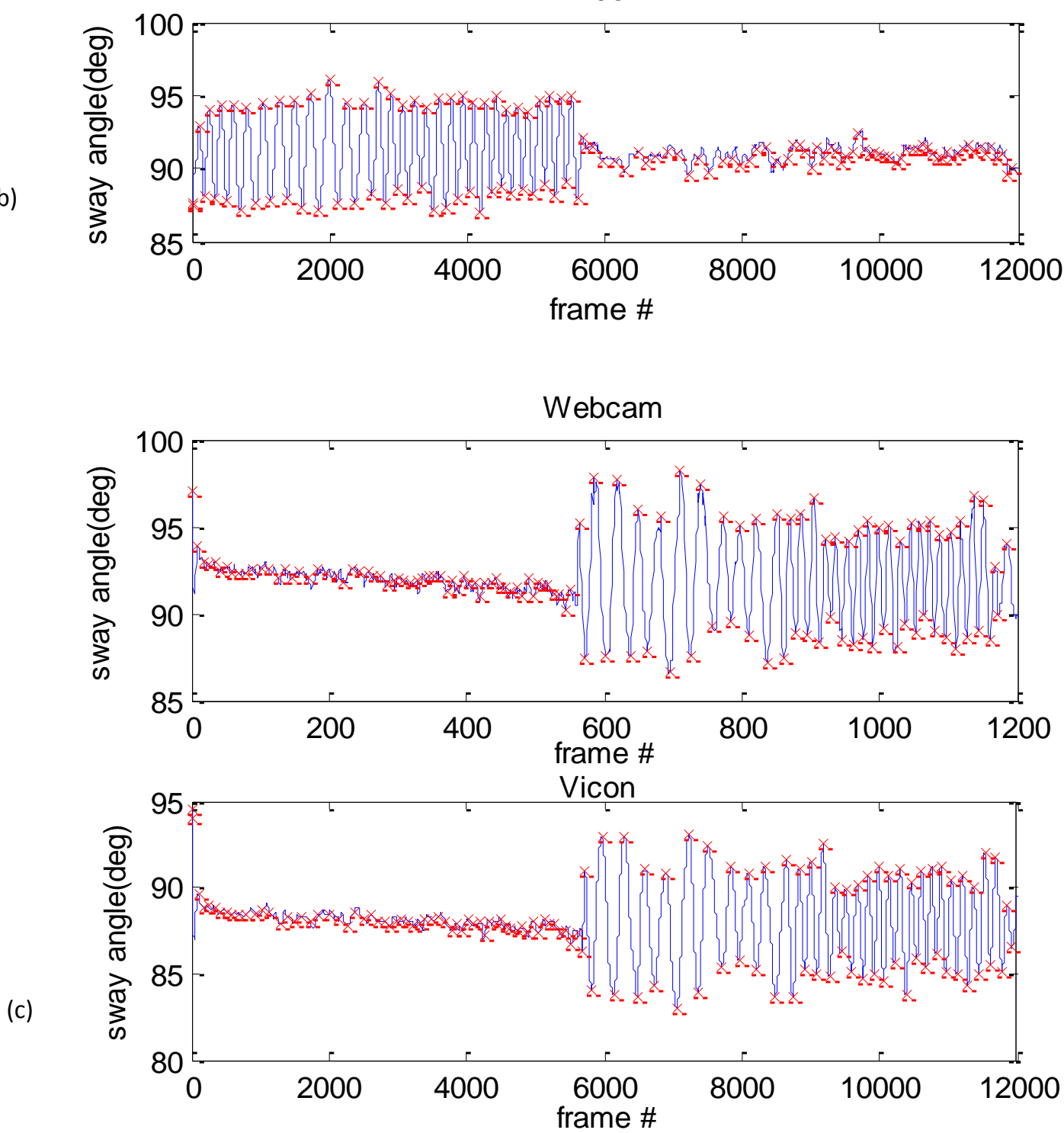

Fig.4.2 Sway comparison during standing: Webcam vs. Vicon (a) Sway amplitude (b) Sway angle vs. X-axis: anterior-posterior sway (c) Sway angle vs. Y-axis: lateral sway 
Table 6. Sway Amplitude Comparison while Standing

\begin{tabular}{|c|c|c|c|c|c|}
\hline $\begin{array}{c}\text { Avg. } \\
\text { sway } \\
\text { amplitude }\end{array}$ & $\begin{array}{c}\text { Webcam } \\
(\mathrm{mm})\end{array}$ & $\begin{array}{c}\text { Vicon } \\
(\mathrm{mm})\end{array}$ & $\begin{array}{c}\text { Avg } \\
\text { diff } \\
(\%)\end{array}$ & $\begin{array}{c}\text { Max } \\
\text { diff } \\
(\%)\end{array}$ & $\begin{array}{c}\text { Std } \\
\text { diff } \\
(\%)\end{array}$ \\
\hline $\begin{array}{c}\text { A-p } \\
\text { sway }\end{array}$ & 140.4 & 154.1 & 8.4 & 16.7 & 9.9 \\
\hline $\begin{array}{c}\text { Lateral } \\
\text { sway }\end{array}$ & 138.0 & 142.9 & 3.1 & 11.4 & 5.1 \\
\hline
\end{tabular}

Table 7. Sway Angle Comparison while Standing

\begin{tabular}{|c|c|c|c|c|c|}
\hline $\begin{array}{c}\text { Avg. } \\
\text { sway } \\
\text { angle }\end{array}$ & $\begin{array}{c}\text { Webcam } \\
(\mathrm{deg})\end{array}$ & $\begin{array}{c}\text { Vicon } \\
(\mathrm{deg})\end{array}$ & $\begin{array}{c}\text { Avg } \\
\text { diff } \\
(\%)\end{array}$ & $\begin{array}{c}\text { Max } \\
\text { diff } \\
(\%)\end{array}$ & $\begin{array}{c}\text { Std } \\
\text { diff } \\
(\%)\end{array}$ \\
\hline $\begin{array}{c}\text { A-p } \\
\text { sway }\end{array}$ & 7.6 & 6.6 & 15.1 & 36.5 & 7.4 \\
\hline $\begin{array}{c}\text { Lateral } \\
\text { sway }\end{array}$ & 7.0 & 6.3 & 11.4 & 32.9 & 6.8 \\
\hline
\end{tabular}

\subsubsection{Sway during Walking}

Fig.4.3(a) overlays a 2-D trajectory of the body centroid for a walk from the webcam and the Vicon system respectively, and Fig. 4.3(b) and (c) show the extracted lateral sway amplitude from the same cameras.

Table 8 shows the lateral peak-peak sway amplitude comparison while walking. We had selected only the experiment samples with a sway amplitude greater than or close to $50 \mathrm{~mm}(\sim 2$ inch). Below this threshold, the voxel person's resolution would not give meaningful results. Table 8 shows that the webcam's sway amplitude results are consistently lower than those from the Vicon system; and the difference in terms of percentage is much larger compared to the standing case. This difference is because, for the standing case, the subject stands in the one optimal reconstruction location with respect to webcam locations, while for walking, the voxel person reconstruction error is 
much larger at boundary locations. In addition, in the standing case, there are many more sway cycles for analysis and with a larger sway amplitude compared to walking, which could eliminate some of the statistical errors and compensate for the lower webcam frame rate and voxel space resolution. Although the webcam results show seemingly large differences from the Vicon results, the differences are in fact all within the 1 inch $(25.4 \mathrm{~mm})$ of the resolution limit, which leads us to believe that it is mainly due to a limitation from the voxel space resolution. Possible solutions include adding extra cameras and increasing camera frame rate and voxel space resolutions. Another alternative would be to use the fuzzy voxel person as a means of reducing reconstruction errors [57].

(a)
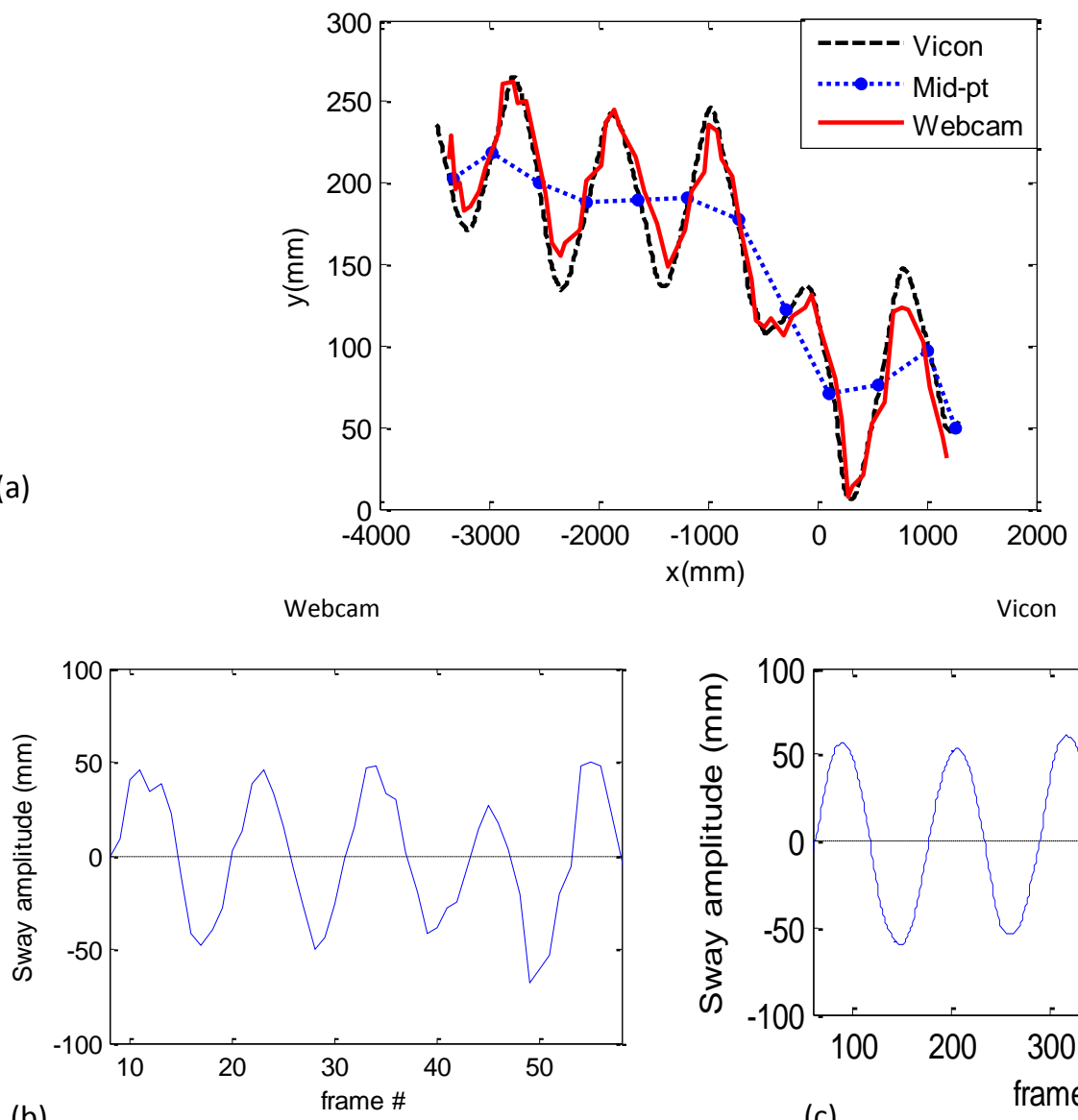

(b)

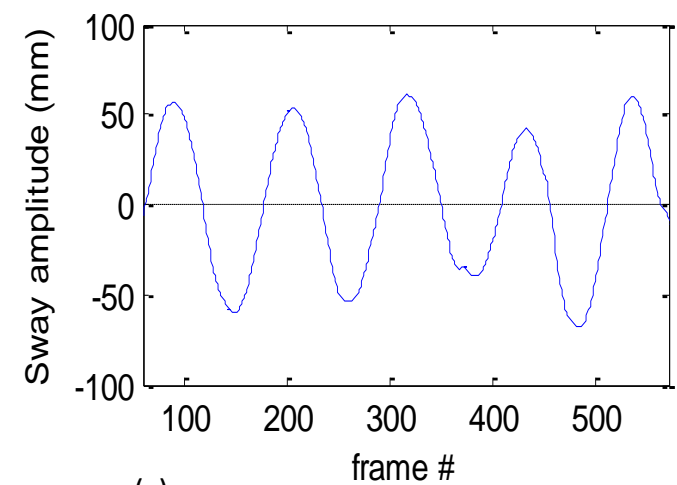

(c)

Fig.4.3 Sway comparison during walking for ID2 slow walk (a) Body centroid 2-D trajectory: Webcam vs. Vicon (b) Webcam: sway amplitude (c) Vicon: sway amplitude 
Table 8. Sway Amplitude Comparison while Walking

\begin{tabular}{|c|c|c|c|c|c|}
\hline & $\begin{array}{c}\text { Webcam } \\
(\mathrm{mm})\end{array}$ & $\begin{array}{l}\text { Vicon } \\
(\mathrm{mm})\end{array}$ & & $\begin{array}{c}\text { Webcam } \\
(\mathrm{mm})\end{array}$ & $\begin{array}{l}\text { Vicon } \\
(\mathrm{mm})\end{array}$ \\
\hline \multicolumn{3}{|c|}{ ID1 } & \multicolumn{3}{|c|}{ ID2 } \\
\hline slow & 98.1 & 101.2 & normal & 53.3 & 65.2 \\
\hline $\operatorname{limp}$ & 81.8 & 91.8 & slow & 93.7 & 109.5 \\
\hline \multicolumn{3}{|c|}{ ID3 } & \multicolumn{3}{|c|}{ ID4 } \\
\hline normal & 65.1 & 74.0 & normal & 42.5 & 50.9 \\
\hline limp & 61.1 & 79.9 & slow & 64.9 & 74.6 \\
\hline
\end{tabular}

\subsection{Validation Experiment for 180 Degree Turn Analysis}

Turn time extracted from the model is compared with the turn time rated by the physical therapy expert in the MU CERT team. Expert scoring started when the participant's first noticeable change in step direction occurs (at foot contact on the floor). Scoring stopped when the participant's foot contacted the floor on the first step back toward the chair, after both feet were facing the intended direction of travel (back to the chair). Expert scoring was chosen over other means, e.g., a pedometer, because of the challenges in determining the start and the end of the turn.

Fig.4.4 and Table 9 show the turn time comparison results. The turn time extracted from the model has a reasonably good match to the expert's rating. The mean difference is $0.11 \mathrm{~s}$, with a maximum of $-0.6 \mathrm{~s}$ and a standard deviation of $0.27 \mathrm{~s}$. We have observed that the difference is mainly caused by the fact that the expert scoring focuses on the foot movement, while the algorithm detects the body centroid change. Many times, the participants' foot started to turn, but the body centroid had not deviated enough from the straight walk to be detected as the start of the turn. 
Similarly, there are cases that the feet appear to exit the turn, but the body is still in the transition stage.

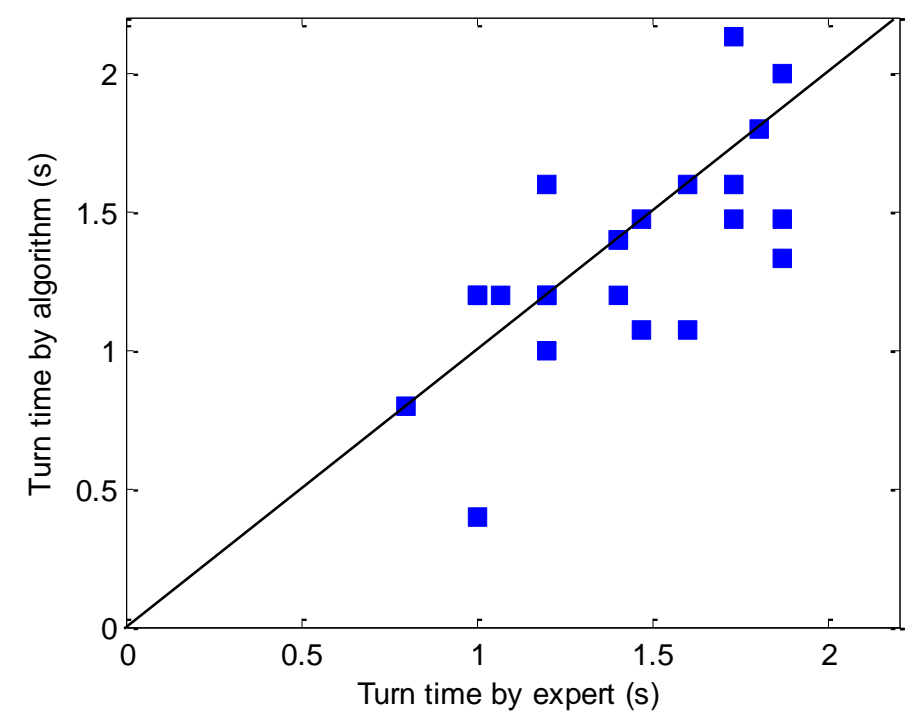

Fig.4.4 Turn time comparison

Table 9. Turn Time Comparison: Model vs. Expert

\begin{tabular}{|l|c|c|c|}
\hline & Avg diff (s) & Max diff (s) & Stdev (s) \\
\hline Model vs. Expert & 0.11 & -0.6 & 0.27 \\
\hline
\end{tabular}

The accurate counting of the turn steps is a challenging problem, due to the various types of turns as well as the low walking speed and irregular steps people take to complete the turn, such as the smaller or shuffling steps used by those with difficulties in turning. Similar challenges in step counting exist for other type of sensor systems in a low speed situation. For example, Traldsen, Askim, Stetvold, Einarsen, Bjastad, Indredavik, \& Helbostad [58], reported that the activPAL Professional single-axis 
accelerometer sensor system underestimated step counts during walking, especially at slow walking speeds below $0.47 \mathrm{~m} / \mathrm{s}$.

In this dissertation's research, the turns can be categorized into three types: (1) pivot turn, (2) pivot step turn, and (3) step turn. Four participants took pivot/pivot step turns, with the majority of them being pivot step turns. The carpet surface floor may be a contributing factor in that subjects could not take complete pivot turns easily. The other three participants took step turns. As mentioned, the floor type can affect how a person turns. A webcam fall risk assessment system should not be affected by the floor type. However, certain types of flooring are more reflective and may cause shadows which can be challenging for accurate silhouette extraction.

The comparison of turn steps rated by the expert and those detected by the algorithm is shown in Fig.4.5, and Table 9. The number of turn steps ranges from 1 to 4 . Normally, more than four steps indicate increased fall risk [59]. Among the total 24 test runs, the detected number of steps in eight runs matched the expert's count, and 16 runs have one step less than the expert's count. In the 16 mismatched cases, 10 of them are pivot/pivot step turns, and 6 are step turns.

The mismatch in the pivot/pivot step turns case is believed to be due to the nature of the pivot step turns. In one type of the pivot step turn, the person pivots with two feet apart. In this case, the distance between the two feet remains almost unchanged during the turn such that the algorithm only detects it as one step. But the expert considers it as two steps because it involves movements of both feet. In another type of pivot step turn, one foot strides first, and the other foot moves next to this foot, then pivots. The expert scores it as two steps, but the algorithm only detects one step when the first foot strides. 


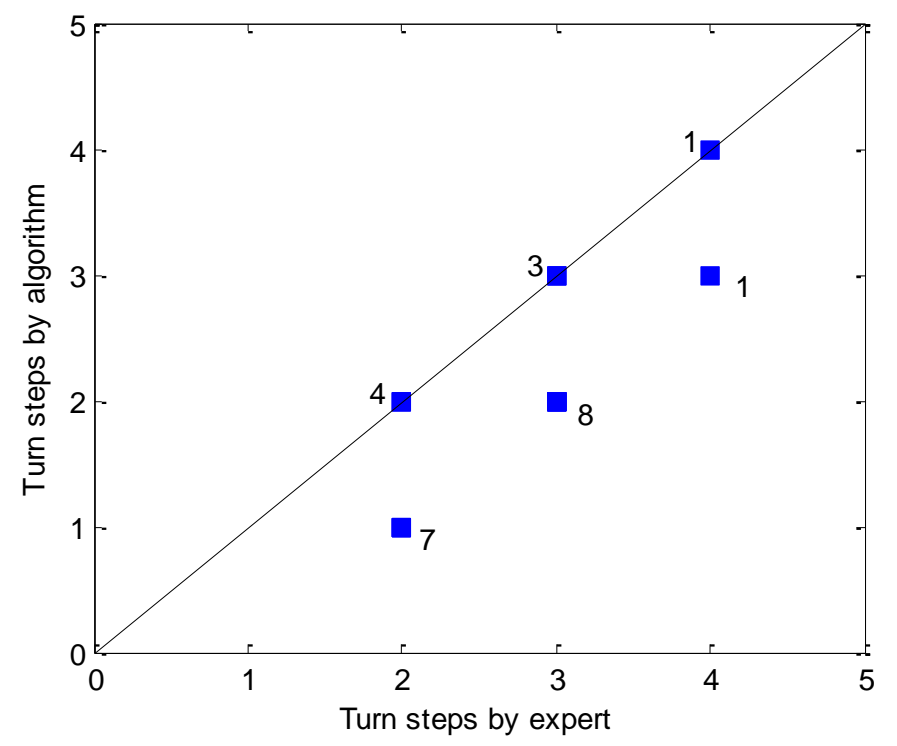

Fig.4.5 Turn step comparison. Number of test runs associated with each data points is listed beside the data point.

Table 10. Turn Steps Comparison

\begin{tabular}{|c|c|c|c|c|}
\hline $\begin{array}{c}\text { Num. of steps } \\
\text { rated by the } \\
\text { expert }\end{array}$ & $\begin{array}{c}\text { Num. of steps } \\
\text { detected }\end{array}$ & $\begin{array}{c}\text { Number of test } \\
\text { runs }\end{array}$ & $\begin{array}{c}\text { Number of step } \\
\text { turns }\end{array}$ & $\begin{array}{c}\text { Number of } \\
\text { pivot/pivot step } \\
\text { turns }\end{array}$ \\
\hline 2 & 2 & 4 & 0 & 4 \\
\hline 2 & 1 & 7 & 0 & 7 \\
\hline 3 & 3 & 3 & 2 & 1 \\
\hline 3 & 2 & 8 & 5 & 3 \\
\hline 4 & 4 & 1 & 1 & 0 \\
\hline 4 & 3 & 1 & 1 & 0 \\
\hline
\end{tabular}

One of the mismatched step turn runs is due to shuffling where the step is too small to be detected under current voxel resolution. The remaining mismatched step turn runs come from the turning time differences between the expert and the algorithm. 


\subsection{Gait Analysis Testing with Elderly Participants $-1^{\text {st }}$ Round Testing}

\subsubsection{Study Overview}

The ultimate goal of the project was to deploy the system in elderly residents' homes. Testing the system in a realistic environment involving elderly residents and getting their feedback is crucial to the real-world application of this research. Ten residents living in independent TigerPlace apartment were recruited to participate in a test and validation of the multi-camera system for gait assessment in scripted scenarios. The ages of the participants range from 81 to 94 years old. Some of them walk independently and some use a walker, cane or wheelchair. The assistive devices impose more challenges to the gait analysis. All participants manage a chronic medical condition; and some have a history of falling. This dissertation will document the results of eight participants who completed all tests. Two of the participants initially recruited were not able to complete all the tests due to health reasons. The residents were first tested on the GAITRite system, to establish ground truth for the camera system, as well as to provide baseline gait information.

All volunteers then participated in two scripted scenarios with realistic daily activities. The scenarios involved a two-person environment designed to include common everyday activities and provide the type of information needed to assess physical functions of older adults.

The first scenario is the visitor scenario in which the resident is sitting in a chair in the living room. The visitor walks into the room to the chair, bends and greets the resident with a hug, and then sits on the couch nearest the resident for two minutes as they talk. The resident then stands up and walks to the kitchen, opens the cabinet, and 
reaches for a cup and tea. He/she pours water into the cup, opens the microwave, and places the cup inside. The resident and the visitor chat as they wait for the tea. The resident then walks back and hands the cup to the visitor. They both sit while the visitor drinks the tea. The visitor then rises from the couch, approaches the side of the chair where the resident is sitting and helps the resident to stand (if assistance is needed). The resident walks to the door, puts on a jacket and opens the door. The visitor exits, and the resident follows.

The second scenario is the housekeeper scenario. In this scenario, an active resident enters the room after grocery shopping. He/she reaches up to open a cabinet, places groceries in, and then walks across the living room, bends and picks up newspapers on a low table, walks across the room to the kitchen recycling receptacle and places them in the receptacle. After pausing briefly, he/she walks back to the living room and opens the door. A housekeeper enters with cleaning supplies. The housekeeper takes some supplies and begins cleaning. The resident sits in the chair and reads for a minute, and then leaves the room. The housekeeper leaves the room after a minute.

The scenarios are explained to each volunteer resident in detail before the recording. A research team member plays the visitor or housekeeper role and is present to provide step by step instructions during the data collection. The residents are not required to memorize any step. Volunteer residents are given gift cards for their participation and seem to enjoy their roles as paid actors. An example of a participant in one of the scenarios is shown in Fig.4.6. 
(a)

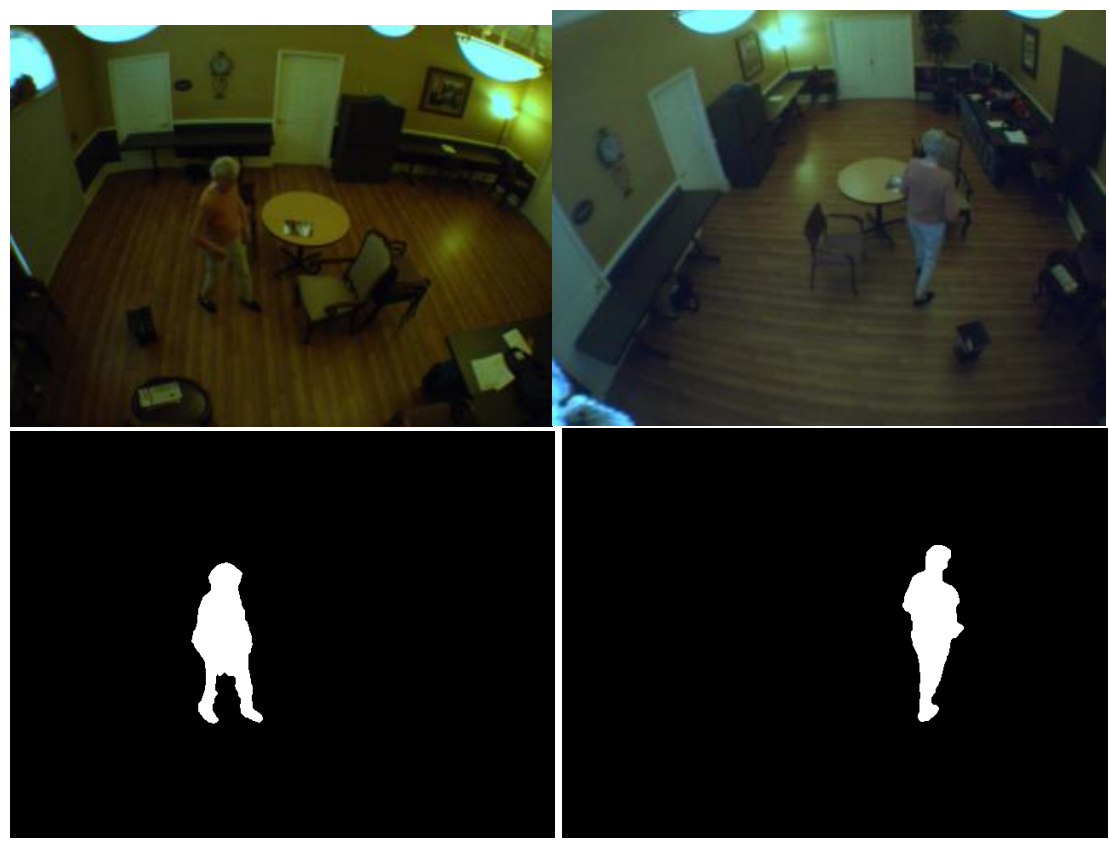

(b)

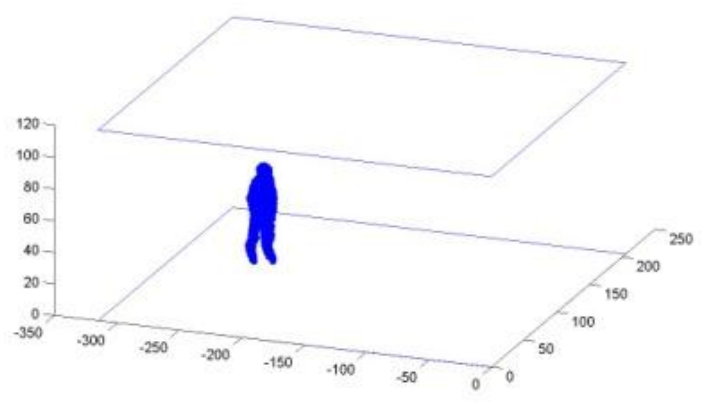

(c)

Fig.4.6 Resident in scenario. (a) Two cameras monitoring the same scene. (b) Extracted human silhouettes. (c) Three dimensional voxel person reconstructed from the silhouettes.

The gait parameters studied include walking speed, step time and step length. The step length asymmetry parameter for these participants was not extracted due to the voxel space resolution discussed earlier. The detailed extraction procedure can be found in Section II. The work described in previous sections outlines the validation conducted in a lab setting with research team members. Here, the work is taken out of the lab into a less structured setting with elderly volunteers. 


\subsubsection{Results}

a) Validation with GAITRite

In order to further validate the system in more realistic daily living settings with elderly residents, the F. Wang research team conducted the following experiments in TigerPlace with elderly volunteers. Participants walked on the GAITRite mat while cameras recorded images. The GAITRite mat was placed in different angles with respect to the cameras to test the camera systems performance under various viewing angles. All achieved good matching results compared to the GAITRite system.

Table 11 presents results for gait parameters extracted from the voxel model compared with the GAITRite system. They are very well matched, considering the camera frame rate and voxel person resolution. The summarized analysis results including maximum, average and standard deviation are also comparable with the ones obtained in the lab settings (Table 1-3). These results verified in this research's camera system performance and accuracy in a realistic daily environment.

Representative GAITRite tests are presented in Table 11 and Fig.4.7 for each participant. In addition to these tests, each participant performed several consecutive tests on the GAITRite. One positive trend for all the participants was that their walking speed gradually increased as the GAITRite tests progressed. It may be that as they became more adept at walking on the GIRTRite carpet, their confidence level increased as fear of falling decreased. . 
Table 11. Cameras (cam) Compared with GAITRite (GR) at TigerPlace with Resident Participants

\begin{tabular}{|c|c|c|c|c|c|c|c|c|}
\hline & \multicolumn{2}{|c|}{ Participant 1} & \multicolumn{2}{|c|}{ Participant 2} & \multicolumn{2}{|c|}{ Participant 3} & \multicolumn{2}{|c|}{ Participant 4} \\
\hline & cam & GR & cam & GR & cam & GR & Cam & GR \\
\hline Walk speed $(\mathrm{cm} / \mathrm{s})$ & 79.8 & 80.5 & 77.3 & 77.1 & 74.6 & 76.9 & 88.4 & 88.2 \\
\hline Step time (s) & 0.66 & 0.68 & 0.58 & 0.57 & 0.67 & 0.65 & 0.58 & 0.58 \\
\hline \multirow[t]{3}{*}{ Step length $(\mathrm{cm})$} & 52.5 & 54.3 & 45.0 & 44.1 & 49.7 & 49.6 & 50.8 & 50.8 \\
\hline & \multicolumn{2}{|c|}{ Participant 5} & \multicolumn{2}{|c|}{ Participant 6} & \multicolumn{2}{|c|}{ Participant 7} & \multicolumn{2}{|c|}{ Participant 8} \\
\hline & cam & GR & cam & GR & cam & GR & Cam & GR \\
\hline Walk speed $(\mathrm{cm} / \mathrm{s})$ & 79.6 & 78.5 & 63.6 & 63.4 & 88.9 & 88.6 & 69.8 & 69.1 \\
\hline Step time (s) & 0.49 & 0.51 & 0.7 & 0.7 & 0.53 & 0.55 & 0.60 & 0.62 \\
\hline Step length $(\mathrm{cm})$ & 39.4 & 39.5 & 44.5 & 44.2 & 47.4 & 48.4 & 41.9 & 42.6 \\
\hline
\end{tabular}
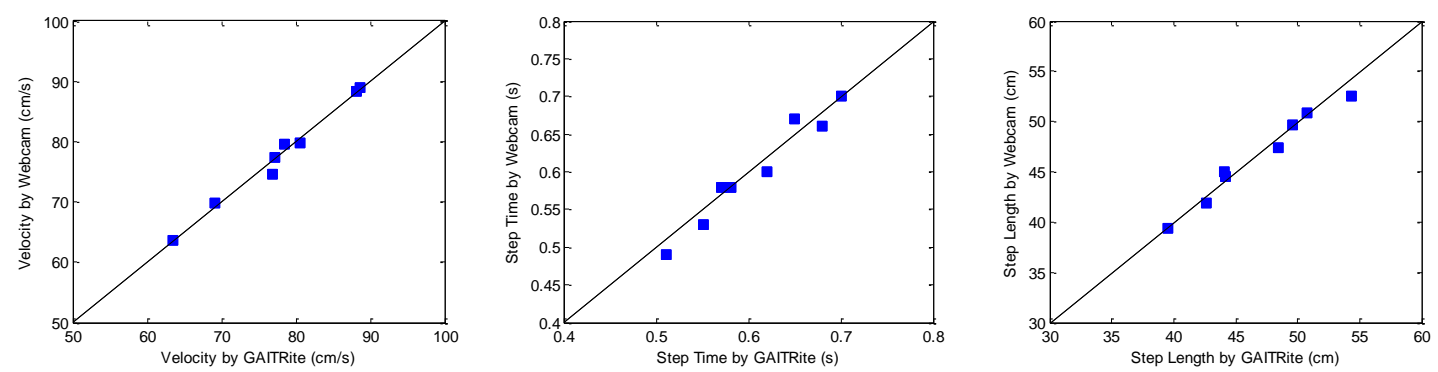

Fig.4.7 Webcam vs. GAITRite for TigerPlace residents

\section{b) Gait Assessment with Realistic Scenarios}

The scenario videos were manually segmented to obtain sections in which the participant has a continuous walking distance with a minimum of 4 steps. One example is shown in Fig.4.8. The walking trajectory in 2-dimensional space is plotted in Fig.4.8 (b). The points represent the centroid locations of the voxel person in each frame. The participant started walking from point $\mathrm{A}$ and ended in point $\mathrm{B}$. The total walking distance is $160.7 \mathrm{~cm}$ and finished with 4 step cycles. The walk path apparently is not a straight walk. By using multiple cameras to reconstruct the 3-D voxel person, the system and 
algorithm were designed to handle such a situation properly. Some voxel person examples are shown in Fig.4.8(a).

In the following section, the cases of the eight participants are presented. The gait parameters extracted from the scenario are compared with the participant's GAITRite results. As mentioned earlier, the GAITRite tests are done separately from the scenario runs, and results listed below in Table 12 are obtained from the average of usually four consecutive GAITRite runs in order to account for the gait variations the participants have displayed.

Through the study, the participants walk patterns fell into two categories-slow and varied. The first category includes participants whose walks are consistently slower, strides are slower and step lengths are smaller compared to the GAITRite runs. This group includes participants 1-5 (Table 12). The data are collected at different times and some at different locations; however, these participants walked consistently faster on the GAITRite.

In the second category, participants displayed a different pattern. Here, all three participants (6-8) have some walks faster than the GAITRite results, and some walks slower (Table 13). In the faster walks, the participants all walked with higher speed, and shorter step time. For example, for participant 6 , walk 2 and walk 3 are taken from the same scenario, only minutes apart, while walk1 is at a different location on a different date. It is interesting to notice that walk 2 is different from walk 1 and walk 3 in terms of speed and step time. The participant walks much faster in walk2 compared to all other walks, including on the GAITRite. Some factors might contribute to the difference. One factor is the walking purpose. In walk2, the participant is going towards a table to pick up 
a newspaper, and in walk3, the participant is going towards a trash can to discard the newspaper. In addition, walk2 $(198 \mathrm{~cm})$ has a longer walk distance than walk1 $(146 \mathrm{~cm})$ and walk3 $(137 \mathrm{~cm})$.

In the test scenarios, five participants have consistently slower walks, with a slower step rate compared to the GAITRite runs. The other three participants walked sometimes faster and sometimes slower than the GAITRite tests. In the 21 walks studied, only 4 walks were faster walks, whereas 17 walks were slower than the GAITRite walks. When considering the difference in walking patterns displayed by the participants between the everyday scenarios and the GAITRite, one factor may be that the scenario's in-home walking distance is usually shorter than the GAITRite $16-\mathrm{ft}(508.8 \mathrm{~cm})$ walkway length that one can use to accelerate. In addition, we also found that the walking pattern can be affected by walking purpose and intention. Another factor might be the Hawthorne effect which is a form of reactivity wherein subjects improve some aspect of their behavior while being measured simply in response to the fact that they are being watched, and not in response to any particular experimental manipulation $[60,61]$. 

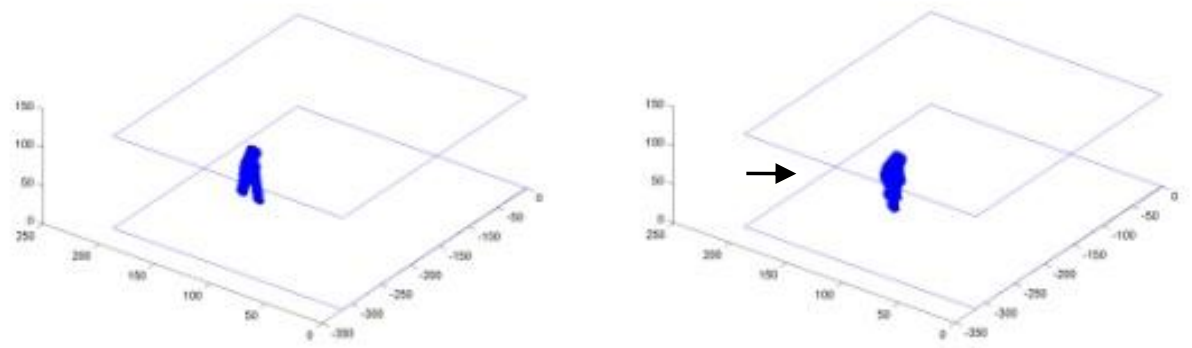

$\downarrow$
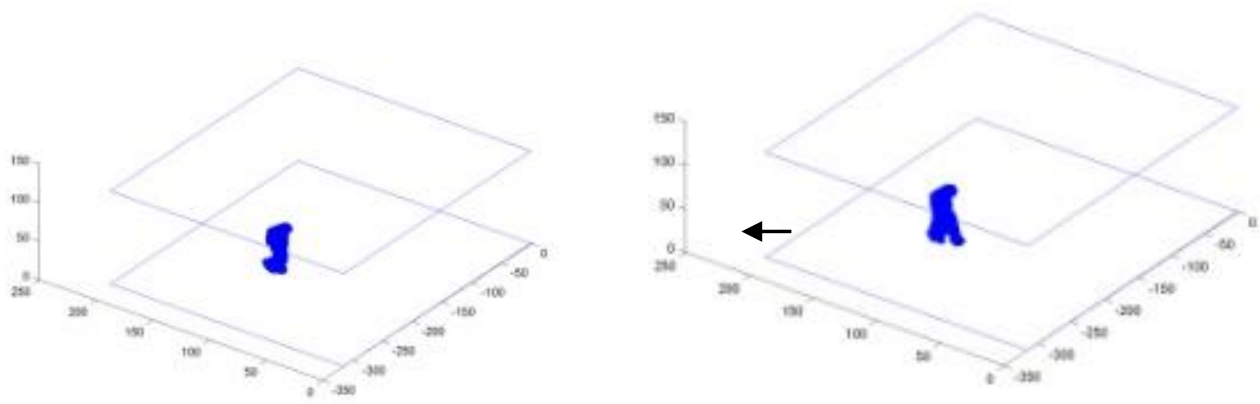

(a)

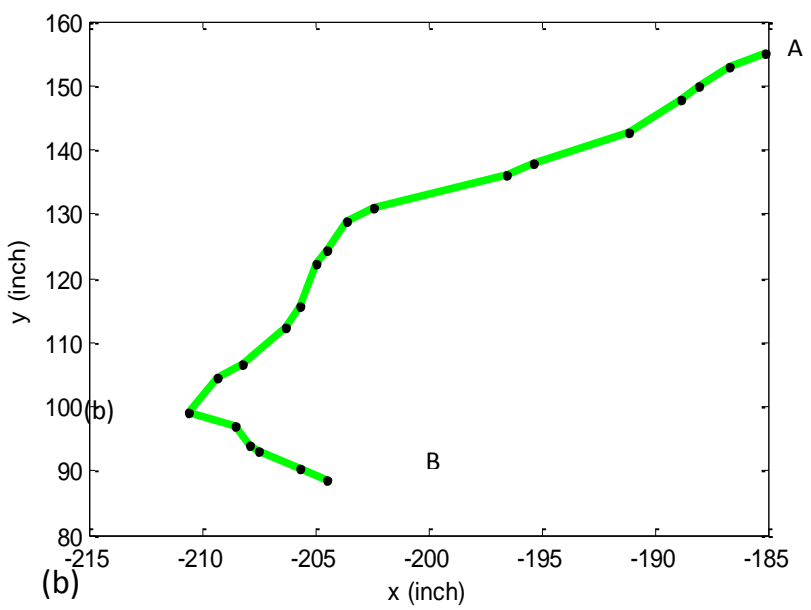

Fig.4.8. Resident in realistic scenario. (a) Sample voxel persons in sequence (b) Walking trajectory projected in 2-D space. Each point represents the centroid location in a frame. 
Table 12. Scenario Walks Compared with GAITRite Tests for Participants 1-5. Participants Walked Faster in the GAITRite Runs.

\begin{tabular}{|c|c|c|}
\hline \multirow{2}{*}{ Participant 1 } & \multicolumn{2}{|c|}{$\begin{array}{c}\text { In-home } \\
\text { scenario results }\end{array}$} \\
\cline { 2 - 3 } & Walk1 & Walk2 \\
\hline Velocity(cm/s) & 59.5 & 34 \\
\hline Step Time(s) & 0.8 & 1 \\
\hline Step Length(cm) & 47.6 & 34 \\
\hline
\end{tabular}

\begin{tabular}{|c|}
\hline $\begin{array}{c}\text { Avg GAITRite } \\
\text { results }\end{array}$ \\
\hline 84.8 \\
\hline 0.65 \\
\hline 54.7 \\
\hline
\end{tabular}

\begin{tabular}{|c|c|c|}
\hline \multirow{2}{*}{ Participant 2 } & \multicolumn{2}{|c|}{$\begin{array}{c}\text { In-home } \\
\text { scenario results }\end{array}$} \\
\cline { 2 - 3 } & Walk1 & Walk2 \\
\hline Velocity $(\mathrm{cm} / \mathrm{s})$ & 67.2 & 68.2 \\
\hline Step Time(s) & 0.65 & 0.60 \\
\hline Step Length $(\mathrm{cm})$ & 43.7 & 40.9 \\
\hline
\end{tabular}

\begin{tabular}{|c|}
\hline $\begin{array}{c}\text { Avg GAITRite } \\
\text { results }\end{array}$ \\
\hline 74.2 \\
\hline 0.58 \\
\hline 42.8 \\
\hline
\end{tabular}

\begin{tabular}{|c|c|c|c|}
\hline \multirow{2}{*}{ Participant 3 } & \multicolumn{3}{|c|}{ In-home scenario results } \\
\cline { 2 - 4 } & Walk1 & Walk2 & Walk3 \\
\hline Velocity $(\mathrm{cm} / \mathrm{s})$ & 57.8 & 60.8 & 61.2 \\
\hline Step Time(s) & 0.70 & 0.60 & 0.6 \\
\hline Step Length(cm) & 40.4 & 36.5 & 36.7 \\
\hline
\end{tabular}

\begin{tabular}{|c|}
\hline $\begin{array}{c}\text { Avg GAITRite } \\
\text { results }\end{array}$ \\
\hline 74.2 \\
\hline 0.58 \\
\hline 42.8 \\
\hline
\end{tabular}

\begin{tabular}{|c|c|c|}
\hline \multirow{2}{*}{ Participant 4 } & \multicolumn{2}{|c|}{$\begin{array}{c}\text { In-home } \\
\text { scenario results }\end{array}$} \\
\cline { 2 - 3 } & Walk1 & Walk2 \\
\hline Velocity $(\mathrm{cm} / \mathrm{s})$ & 54.3 & 63.6 \\
\hline Step Time(s) & 0.68 & 0.6 \\
\hline Step Length $(\mathrm{cm})$ & 36.9 & 38.2 \\
\hline
\end{tabular}

\begin{tabular}{|c|}
\hline $\begin{array}{c}\text { Avg GAITRite } \\
\text { results }\end{array}$ \\
\hline 92.1 \\
\hline 0.57 \\
\hline 52.3 \\
\hline
\end{tabular}

\begin{tabular}{|c|c|c|}
\hline \multirow{2}{*}{ Participant 5 } & \multicolumn{2}{|c|}{$\begin{array}{c}\text { In-home } \\
\text { scenario results }\end{array}$} \\
\cline { 2 - 3 } & Walk1 & Walk2 \\
\hline Velocity $(\mathrm{cm} / \mathrm{s})$ & 55.1 & 56.1 \\
\hline Step Time(s) & 0.6 & 0.6 \\
\hline Step Length $(\mathrm{cm})$ & 33.1 & 33.7 \\
\hline
\end{tabular}

\begin{tabular}{|c|}
\hline $\begin{array}{c}\text { Avg GAITRite } \\
\text { results }\end{array}$ \\
\hline 68.3 \\
\hline 0.53 \\
\hline 36.1 \\
\hline
\end{tabular}


Table 13. Scenario Walks Compared with GAITRite Tests for the Participants 6-8.

\begin{tabular}{|c|c|c|c|}
\hline \multirow{2}{*}{ Participant 6 } & \multicolumn{3}{|c|}{ In-home scenario results } \\
\cline { 2 - 4 } & Walk1 & Walk2 & Walk3 \\
\hline Velocity $(\mathrm{cm} / \mathrm{s})$ & 66.6 & 82.7 & 62.4 \\
\hline Step Time $(\mathrm{s})$ & 0.73 & 0.60 & 0.73 \\
\hline Step Length $(\mathrm{cm})$ & 48.8 & 49.6 & 45.8 \\
\hline
\end{tabular}

\begin{tabular}{|c|}
\hline $\begin{array}{c}\text { Avg GAITRite } \\
\text { results }\end{array}$ \\
\hline 70.2 \\
\hline 0.67 \\
\hline 46.8 \\
\hline
\end{tabular}

\begin{tabular}{|c|c|c|}
\hline \multirow{2}{*}{ Participant 7 } & \multicolumn{2}{|c|}{ In-home scenario results } \\
\cline { 2 - 3 } & Walk1 & Walk2 \\
\hline Velocity $(\mathrm{cm} / \mathrm{s})$ & 94.9 & 76.1 \\
\hline Step Time(s) & 0.5 & 0.52 \\
\hline Step Length $(\mathrm{cm})$ & 47.5 & 39.6 \\
\hline
\end{tabular}

\begin{tabular}{|c|}
\hline $\begin{array}{c}\text { Avg GAITRite } \\
\text { results }\end{array}$ \\
\hline 87.4 \\
\hline 0.53 \\
\hline 46.6 \\
\hline
\end{tabular}

\begin{tabular}{|c|c|c|c|c|}
\hline \multirow{2}{*}{ Participant 8 } & \multicolumn{4}{|c|}{ In-home scenario results } \\
\cline { 2 - 5 } & Walk1 & Walk2 & Walk3 & Walk4 \\
\hline $\begin{array}{c}\text { Velocity } \\
(\mathrm{cm} / \mathrm{s})\end{array}$ & 60.6 & 76.8 & 66.7 & 72.7 \\
\hline Step Time(s) & 0.7 & 0.57 & 0.73 & 0.53 \\
\hline Step Length $(\mathrm{cm})$ & 42.4 & 43.5 & 48.9 & 38.5 \\
\hline
\end{tabular}

\begin{tabular}{|c|}
\hline $\begin{array}{c}\text { Avg GR } \\
\text { results }\end{array}$ \\
\hline 63.1 \\
\hline 0.69 \\
\hline 43.0 \\
\hline
\end{tabular}

\subsection{Gait Analysis Testing with Elderly Participants $-2^{\text {nd }}$ Round Testing}

\subsubsection{Study Overview}

Based on the observation of the first data set collected in scripted scenarios with elderly residents in TigerPlace (section 4.4), it appeared the scenario's design involved a lot more sitting rather than the walking/moving activities pertinent to this study. Therefore, a new scenario was designed which is more suitable for this dissertation's motion analysis study.

Again, 10 residents at TigerPlace were recruited to participate in a test and validation of the multi-camera system for gait assessment. Six of them walk independently and four 
(ID1, 2, 4, 5) use a cane. This study intentionally omitted people who use more complicated assistive devices such as a walker or wheelchair because of image processing complexity necessitated by such devices. Each participant had two runs of the same scenario.

Since the multi-cameras system was validated by this research both in lab and in daily living environment as described in earlier sections, the F. Wang team proceeded with data collection of scripted scenarios in this round of data collection. Furthermore, participants were given only one scenario this time to meet time restraints and improve percentage of data acquisition needed from each participant with a goal of $100 \%$.

The scenario, described as repairman scenario, has the resident enter the room from outside, walk into the middle of the room, and then remembers to pick up the paper. $\mathrm{He} / \mathrm{sh}$ then returns to the door, takes the newspaper, and comes back to the middle of the room. The resident sits down in a chair, and starts to read newspaper for 10 seconds, but remembers glasses. He or she stands up, goes to get the glasses, and comes back to sit down in the chair. The resident reads newspaper for 10 seconds; and then stands up and comes to the middle of the room maneuvering in both forward and lateral directions. The repair person knocks on the door and comes in. The resident stands up and chats with the repair person for 30 seconds. The repair person puts down his tool box, and goes to check the window that needs to be fixed. Resident steps over and step around the tool box. 


\subsubsection{Results}

\section{a) Comparison with Expert's Rating}

A 10-ft. region was marked on the floor during the data collection. The recorded videos were later evaluated by a physical therapy expert. Using this $10 \mathrm{ft}$. region and a $1 / 100$ accuracy stop watch, the expert timed a section of the video where the participant walks in this 10-ft. region. The expert reported the time and number of steps each participant used to complete the 10-ft. walk. Gait parameters rated by the expert are computed as the following: the velocity equals distance (10 feet)/time; step time is calculated as time/(number of steps); and step length is calculated as distance (10 feet)/(number of steps) or the product of velocity and step time. For the webcam results, F. Wang manually segmented the videos that covers only the 10 -ft. region, and fed those images to the algorithm which reported the same gait parameters to compare with the expert's rating. The three gait parameters are compared in Fig.4.9 and Table 14. The results presented here showed that the velocity has a very close match with the expert rating, despite the velocity's large range of 30-100 cm/s. The largest discrepancy between the gait analysis algorithm and the expert's rating in velocity is in the fastest speed case, where the gait analysis algorithm reported $112.1 \mathrm{~cm} / \mathrm{s}$, while the expert reported 98.6 $\mathrm{cm} / \mathrm{s}$. Since it's the fastest velocity among all tested, it took the shortest time to complete the 10 -ft. distance. Therefore, in this case, the difference is possibly caused by human response time or error when recording for short time period time.

The step time has a reasonably good match with the expert's rating. The number of steps reported by the algorithm matched the expert's results exactly. Therefore, the discrepancy comes from the time difference between the expert's stop watch timing and 
the manual video segmentation. It has been observed that the manually recorded segmentation time was longer than the expert stop watch timing in all except one test. One of the possible reasons is that the algorithm can only recognize full gait cycles, meaning the foot needs to complete both swing and touching the ground stages in order to be recognized as a step. Therefore, Wang had to add one or two extra frames for the foot to complete its gait cycle, which can cause the results from the algorithm to have extra time. To not add the extra frames could cause algorithm results to miss a full step number causing an even larger time discrepancy between manual video segmentation time and stop watch time. The expert however, doesn't have this restriction, and would cut the time as the person passed the finish line of the 10 - $\mathrm{ft}$ region.

Since the step length is computed as the product of the velocity and step time, the discrepancy for this parameter is simply the addition of the difference of the velocity and step time.

A t test was performed on the gait parameter results obtained from the webcam and the expert. The results show that $p>0.05$ for walking speed, meaning the difference between the webcam and the expert is not significant, whereas $p<0.005$ for both step time and step length, meaning the difference is highly significant. It is not surprising to see significant difference in step time and step length, considering the difference in timing discussed earlier. One possible improvement for a more accurate comparison is that the expert could estimate a fraction of a step, instead of the whole step when it is not a full gait cycle. 


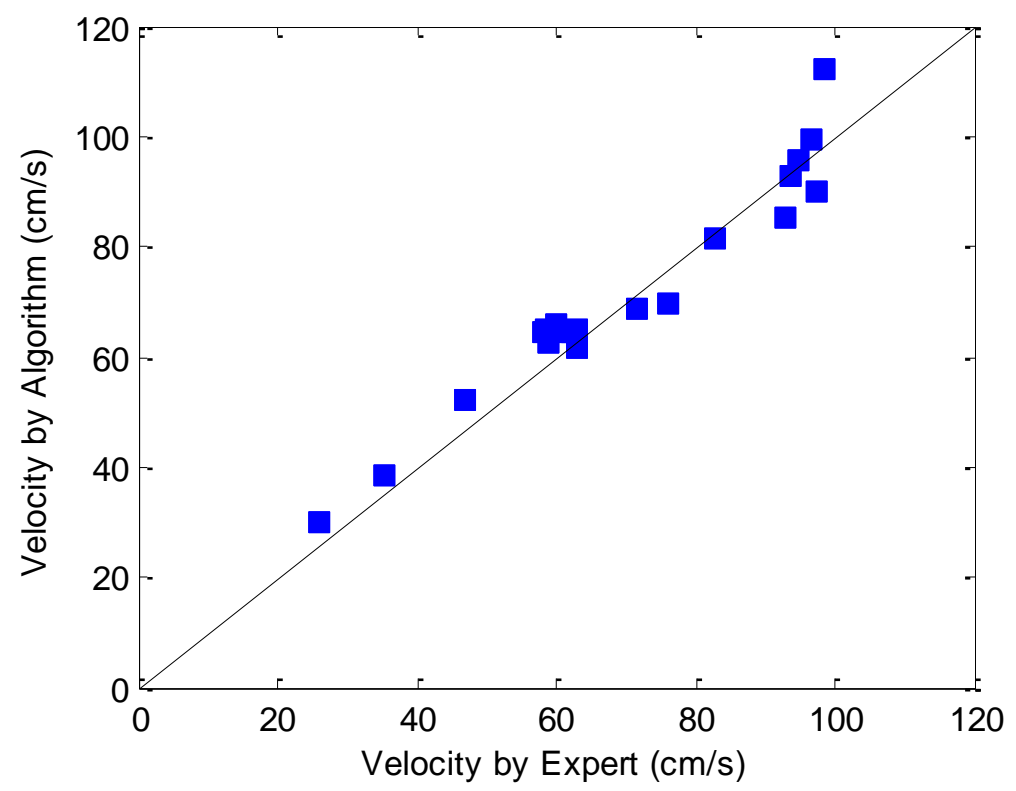

(a)

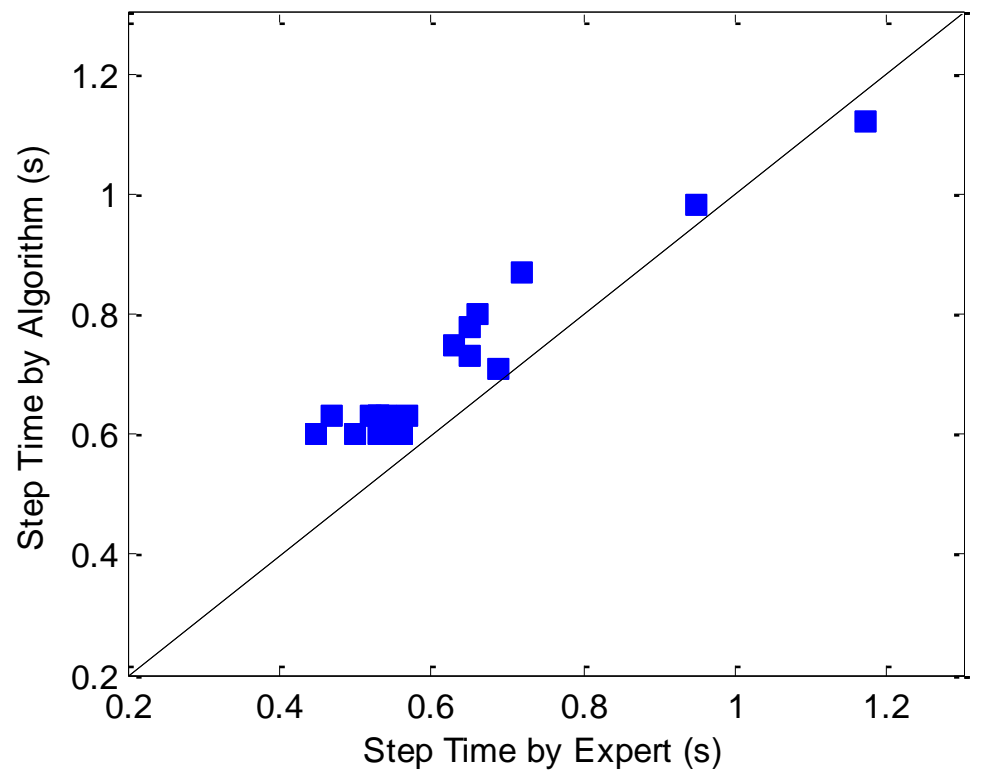

(b) 


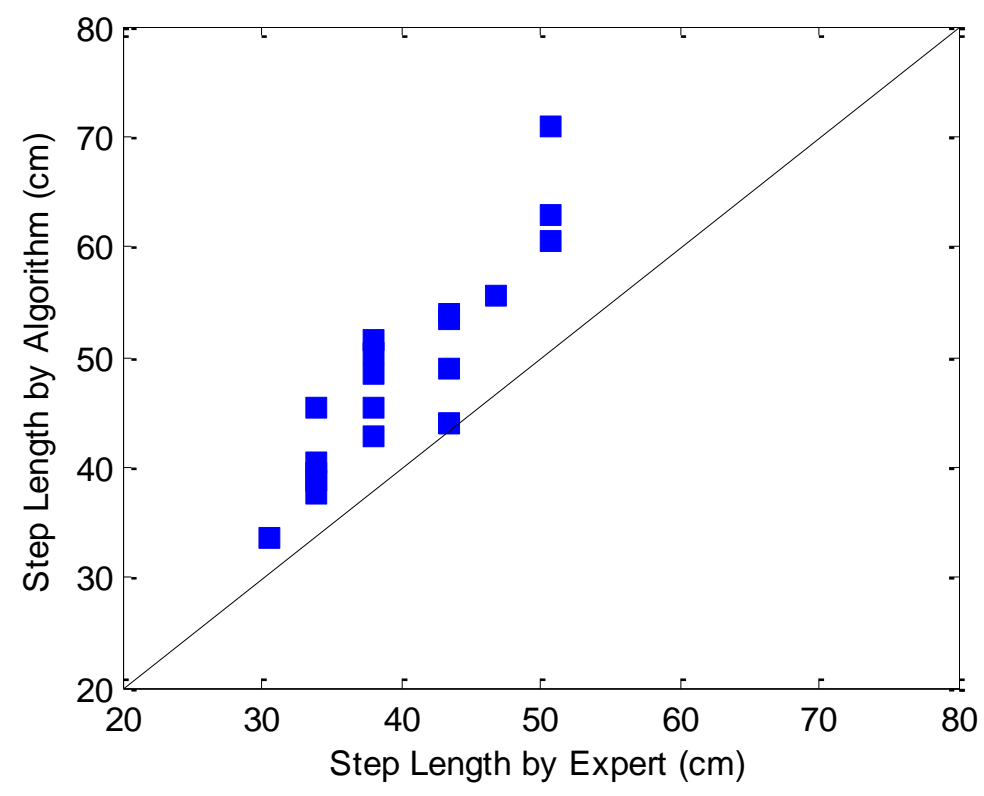

(c)

Fig.4.9 Gait parameters extracted by the algorithm are compared with the expert rating.(a) Velocity (b) Step time (c) Step length

Table 14 Camera vs. Expert Comparison for 10 Feet Walk at TigerPlace

\begin{tabular}{|c|c|c|c|c|}
\hline & avg diff $(\%)$ & $\max \operatorname{diff}(\%)$ & stdev & $p$ value \\
\hline Velocity & 4 & 15 & 7.5 & 0.19 \\
\hline Step Time & 16 & 34 & 9.6 & $3.9 \mathrm{e}-7$ \\
\hline Step Length & 20 & 40 & 10.6 & $4.3 \mathrm{e}-7$ \\
\hline
\end{tabular}

\section{b) Gait Analysis Summary}

The gait analysis results of the 10 participants are shown in Fig.4.10 which includes average velocity, step time, step length, lateral sway and their standard deviation. The average and standard values of the gait parameters are calculated using the gait parameters extracted in each walk segment during one test. The test results are fairly consistent between the two test runs. Putting a person's gait parameters together makes it easier to obtain the whole picture of the person's gait status and to screen out the elderly 
who are at risk of falling. The gait analysis assessment tools developed in this research also make it possible to monitor the elderly for longer time periods and determine the trend of their gait characteristics. Recent study results show that there is significant correlation between walking speed and physical function, and it is reliable to use walking speed as a sole surrogate of the assessment of physical function in the elderly [62]. In addition, a study by Barak et al. [63] shows that, in all speed conditions, the fallers had significantly higher stride frequency(shorter stride time) compared with the nonfallers. The nonfallers had longer strides at all speed conditions compared with the fallers. At most speed conditions, the nonfallers tended to have a wider mediolateral sway; both faller and nofaller groups showed a lateral sway increase with increasing walking speed.

The walking speed for the ten elderly participants falls between 34.8 to $74.5 \mathrm{~cm} / \mathrm{s}$. ID 1 , who uses a cane when walking, clearly walks much slower $(34-40 \mathrm{~cm} / \mathrm{s})$ than the rest of the participants whose walking speed are above $50 \mathrm{~cm} / \mathrm{s}$. In addition, his walking speed has little variation which means that he consistently walks slowly, or in another words, he is not able to walk faster. In addition, this participant's step time is also slower and step length is shorter compared to others tested. His lateral sway amplitude is the lowest, which can be related to his slow walking speed. Generally, people have larger lateral sway when they walk faster than when they are slow. ID2 and ID6 are the two participants who walk fastest, and also they have relatively larger sway amplitude. All ID1's gait parameters imply he might have more difficulties in walking. Even though there is no one in the tested group with a similar walking speed as ID1 to compare with, as suggested in $[62,63]$, low walking speed, short stride length, and low lateral sway are 
all warning signs of a faller, or someone at higher risk of falling, and may need medical intervention.

(a) Velocity
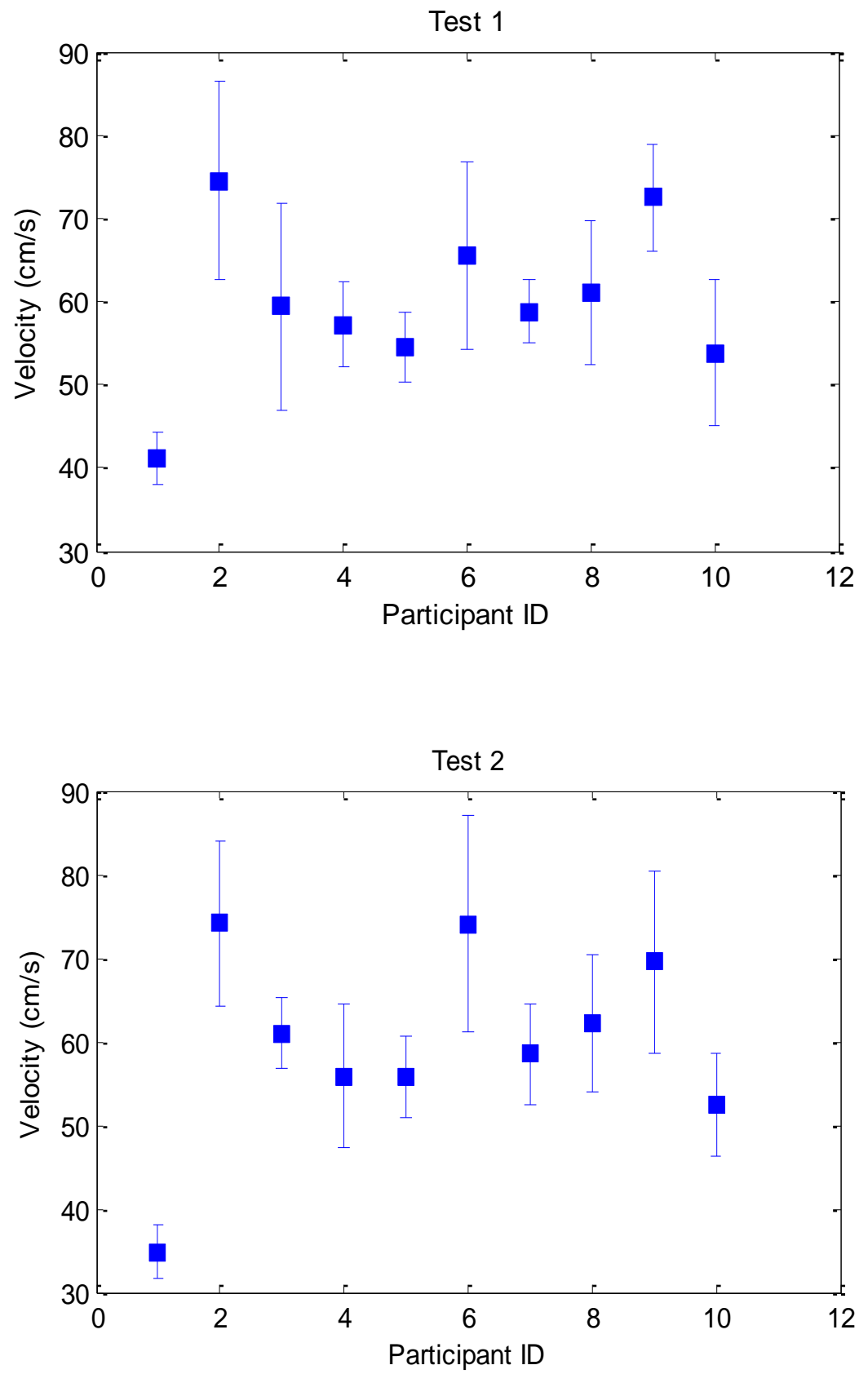
(b) Step Time
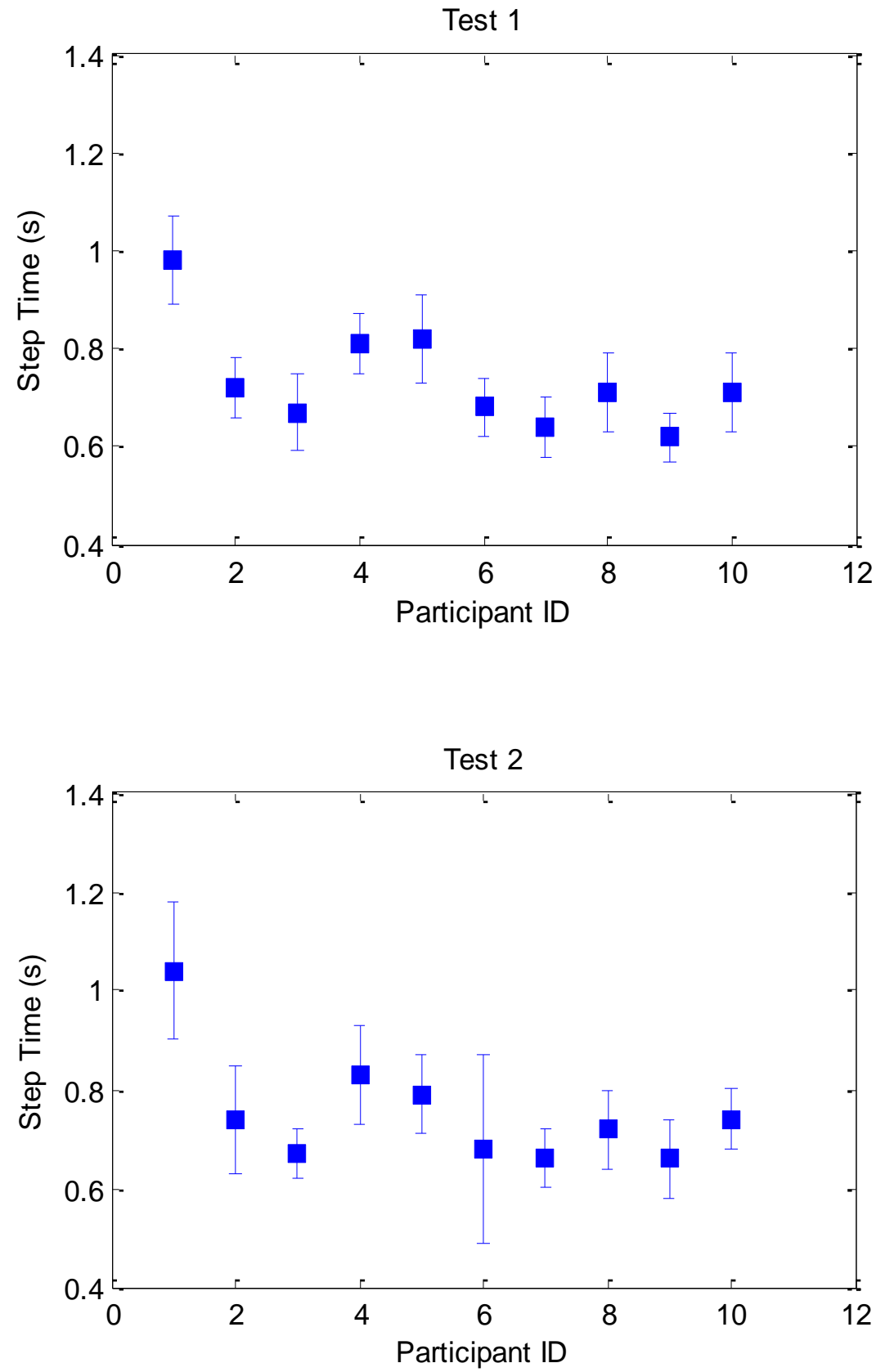
(c) Step Length
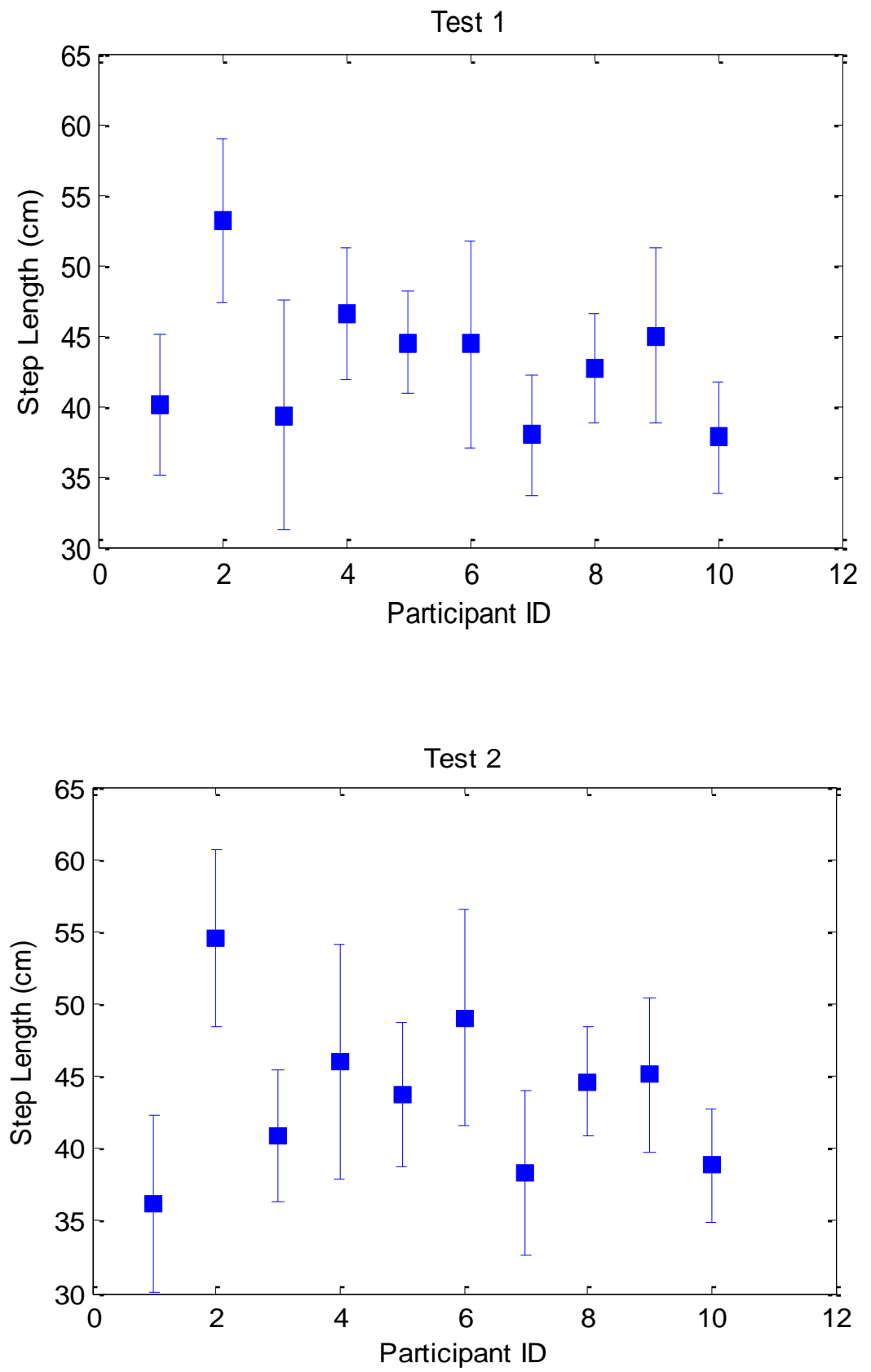
(d) Lateral Sway Amplitude
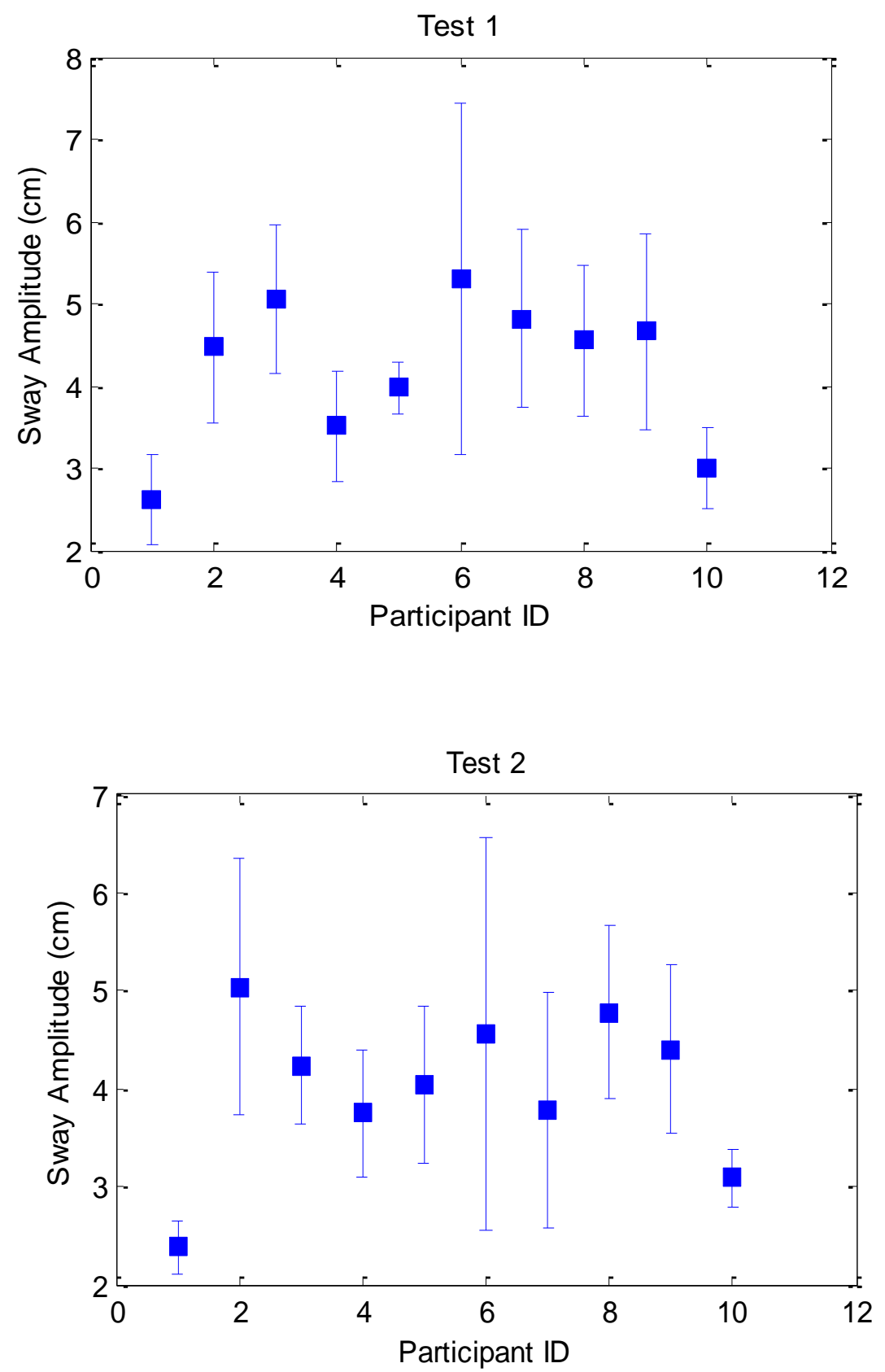

Fig.4.10 Gait Analysis Results for 10 elderly participants. The gait parameter average values and their standard deviation (a) Velocity (b) Step Time (c) Step Length (d) Lateral sway amplitude 


\section{Chapter 5. Discussion}

The main goal of this dissertation is to investigate the feasibility of developing and eventually deploying such vision technology for eldercare gait assessment. Even though the basic concept and techniques of voxel reconstruction from silhouettes using multiple cameras have been developed for a long time, this study is the first application using computer vision techniques for continuous monitoring of older adults' gait assessment in an unstructured dynamic daily living environment. The study is well designed and executed to validate the algorithms developed, not only in lab settings but also in a scripted, realistic home setting. The realistic scenario testing took place in the retirement community, and was carefully designed to include common everyday activities that not only are performed on a daily basis by elderly residents, but also provide the type of information needed to assess physical functions of older adults. This extensive evaluation has provided realistic testing of the system in preparation for deployment in the homes of elderly residents.

The system algorithms presented in this dissertation are compared to ground truth, including a Vicon motion capture system, a GAITRite mat and a physical therapy expert, in order to understand how well this system measures up to the gold standard in this domain. Our results show that the difference is not significant $(p>0.05)$ in walking speed, step time and step length between the webcam system and the GAITRite; the difference is significant $(p<0.02)$ when comparing step time between the Vicon and the webcam system or the GAITRite, but not significant $(p>0.05)$ for walking speed and step length. The system was also compared with the expert rating in the realistic scenario testing. There is no significant difference $(p>0.05)$ in walking speed between the system and the 
expert, but highly significant $(p<0.005)$ difference in step time and step length. This could be explained by a timing difference caused by the algorithm and the expert as mention in section 4.5.2.There is a clear need for more investigation in terms of the time difference observed between the webcam system and the Vicon, and more so between the webcam system and the expert. Through all these observations, it is not difficult to see that velocity or walking speed is observed to be the most robust and reliable gait parameter in all of the validation experiments. Recent study results show that there is a significant correlation between walking speed and physical function, and it is reliable to use walking speed as a sole surrogate of the assessment of physical function in the elderly [62].

Under the current resolution limited by hardware and software, it has been demonstrated that the technology can detect different gait characteristics as illustrated in the $2^{\text {nd }}$ round of TigerPlace testing. We are able to obtain the walk to walk variation. It is not necessary to get the finest detail of the human body; the current resolution is adequate for capturing gait changes in the home. However, improvements in resolution will definitely help.

The importance and necessity of continuous in-home gait and balance monitoring is re-affirmed through this study. It has been observed that many of the elderly participants in the study ( 15 out of 20 walking sequences in the $1^{\text {st }}$ round of testing) have displayed different gait patterns during the realistic scenario than when tested on the GAITRite. Even though the data pool is quite limited, the trend should not be overlooked.

Extensive testing with targeted elderly people also provided an important opportunity to get their feedback about the monitoring methods. Video sensors provide a rich source 
of information that can be used for gait analysis; however, it also raises concerns in privacy when placed in personal living environments. The MU Center for Eldercare and Rehabilitation Technology (CERT) team conducted specific studies in TigerPlace which served as the basis for this dissertation. The aim was to utilize CERT and TigerPlace resources to investigate the feasibility of eldercare monitoring applications that use video systems and to facilitate an exploration of older adults' privacy considerations for this type of system. This dissertation's primary research priority was to introduce MU's own innovative and unique voxel reconstruction system and vision-based tools and identify whether distorting or "anonymizing" captured images affect older adults' privacy concerns and willingness to allow such an application to be installed in their residence. Follow-up in-depth interviews with the ten participants, who were recruited to participate in the 'visitor' and 'housekeeper' scenarios were conducted after a demonstration of the captured images. Findings indicate that shape extraction can alleviate privacy concerns associated with the use of cameras. Participants expressed no privacy concerns with silhouette images but emphasized the importance of anonymity in the video sequences. They furthermore expressed the desire to control system operation by being able to turn a vision-based system off and on and also to determine who has access to the collected information [64]. These findings are very important for the development of this technology as it lays the foundation of acceptance by its intended customers.

These positive results demonstrate the feasibility of the proposed methodology. It also addresses several deficits in the current paradigm of gait and balance assessment performed in clinic settings. First, with this system, the gait parameters can now be monitored continuously over time without expensive and inconvenient clinic visits. 
Therefore, elderly who are in high-risk groups can be monitored more closely and rapidly. Second, researchers, health professions and caregivers can have access to more frequent measurements of gait and balance, and the test results can be shown in clear graphs or tables as demonstrated in the dissertation. It could provide an opportunity for early change detection, as well as correlating the gait information with other health conditions. Finally, large scale data collection and analysis of multiple subjects can be performed relatively easily with the aid of this technology, which may lead to new areas of population-based research and diagnostic ability using gait information.

There are still constraints to be considered for this system. The in-home gait assessment tool proposed in this research is a complex system with many features such as: silhouettes extraction, voxel reconstruction, human tracking, activity segmentation and gait analysis. Each component in the system is important and will have far-reaching effects on the next generation results. Furthermore, each component could stand alone as a research topic. This dissertation is not meant to cover every component of this system, but it does cover two-person tracking and simple activity segmentation, but mostly focuses on the motion analysis. The results presented in the dissertation are based on good silhouette extraction and voxel reconstruction. The daily living environment is more challenging compared with the scripted scenarios, which could affect the quality of the silhouettes. With further development of Stone's dynamic background update method [65] and MU's human detector [52], the system can become more robust to changing light condition, moving objects, occlusion etc., and perform well when deployed in unstructured home settings. 
As mentioned earlier, we had intentionally eliminated the elderly residents at TigerPlace who used a walker or wheel chair from the $2^{\text {nd }}$ round data collection. It is clear that complicated assistive devices will affect greatly the algorithm performance. However, velocity as the most robust gait parameter could still be good enough for estimating the moving speed of the person with the assistive devices.

The voxel resolution ( 1 inch used in this dissertation), and the webcam's frame rate (5fps) limit the detection resolution related to step length, step time and body sway, such as the right/left foot difference in these parameters. It could also affect the detection of changes in these parameters until it is above the resolution. With continuous improvement in hardware and software, such as a higher frame rate and higher voxel resolution, accuracy can improve which could lead to earlier and more accurate gait problem detection before a fall occurs.

There are still some challenges facing the broad deployment of this system. One of the great challenges is human identification, meaning accurately identifying the person to be monitored. In fact, it is a common issue facing many passive sensing systems, such as the OHSU PIR system used for capturing walking speed [22]. The common approach is to use sensor fusion, such as a wearable RFID tag. In our study, at the initial stage of the deployment, the system could be limited to single resident rooms to avoid the multiple person identification issue. Future research could address this issue using features extracted from silhouettes and voxel models.

Another challenge important component of the system is the accurate segmentation of the video sequences. When used for continuous monitoring, there are numerous images, and there is a clear need to identify the video segment suitable for gait analysis. 
In this dissertation, a preliminary study was made of sequence segmentation for the scenarios captured. However, this will most likely require further investigation for use in unstructured home environments.

Overall, the results obtained so far are very promising for the development and deployment of this system to elderly housing, whether independent, assisted living or skilled nursing care. 


\section{Chapter 6. Conclusion}

\subsection{Summary}

This dissertation has described motion analysis for gait and balance assessment for the elderly using multiple webcams, and a 3-D reconstructed voxel person from silhouettes. The gait parameter results are validated in lab settings as well as in a realistic retirement community setting. Excellent matching results were achieved when compared with the Vicon motion capture system, the GAITRite mat, and a physical therapist expert, which has provided confidence in the system performance. The various gait parameters extracted provide a detailed gait profile and can be used to screen out people at risk of falling. The system proposed here is inexpensive and has good accuracy verified by ground truth. The passive sensing nature of the system makes it ideal for long term, inhome gait monitoring to detect gait changes.

The contributions of this dissertation are summarized below.

Engineering contributions:

- Development of gait analysis algorithms, including gait parameters, body sway and 180 degree turn around

- Development of a two person tracking algorithm for an in-door environment

- Automatic video segmentation to identify good image sequences for gait analysis, and possible turn around frames.

Application contributions:

- Extensive in-lab testing of the system; 21 participants, over 70 test runs. 
- Extensive realistic environment testing of the system; 20 residents from the retirement community participated in the test with a total of 116valid test runs.

- Data processing and analysis of all the data collected, providing an investigation of the feasibility for vision-based gait and balance monitoring in the home.

\subsection{Future Work}

The capabilities of this system are significant, but several improvement and additions could be made. The first of these would be the more accurate turn around analysis. Although the current algorithm has good detection for possible turn around frames in realistic scenarios, it is still difficult to obtain an accurate turn-around time and step count, especially in a realistic environment due to the noisy body centroid data.

The second important aspect of the system needing further investigation is a more robust and reliable video segmentation algorithm, so that only suitable images are fed to the gait analysis stage of the system.

Another extension to this work would be to have the webcams installed in older adults home and obtain longer term monitoring data for further analysis. One possible research area is to not only obtain the variability of gait parameters over short term periods such as from walk to walk as done in this dissertation, but also the variability in longer time intervals, such as monthly, quarterly and yearly. 


\section{APPENDIX}

\section{List of code and datasets}

All data and code used in this dissertation can be found on the CERT server kronos:

1) home/fwnf5/dissertation_code_data/code

This directory contains the code and a 'README' file which lists the function of each program, input, output, and how to run it.

2) home/fwnf5/dissertation_code_data/data

This directory contains the dataset used in the dissertation, and a "README" file
a. in_lab_data
b. TP_1st
c. TP_2nd

3) home/fwnf5/dissertation_code_data/dissertation.pdf 


\section{BIBLIOGRAPHY}

[1] J. C. Close, S. L. Lord, H. B. Menz, and C. Sherrington, "What is the Role of Falls?" Best Proct Res Clin Rheumatol, vol. 19, no. 6, pp. 913-935, 2005.

[2] R. W. Baloh, S.H. Ying, and K. M. Jacobson, "A Longitudinal Study of Gait and Balance Dysfunction in Normal Older People," Arch of Neurol, vol. 60, no. 6, pp. 835-839, 2003.

[3] M. E. Tinetti, T. F. Williams, R. Mayeski, "Fall Risk Index for Elderly Patients Based on Number of Chronic Disabilities,"The American Journal of Medicine, vol.80, pp. 429-434, 1986.

[4] K. Berg, S. Wood-Dauphinee, J. I. Williams, and D. Gayton, "Measuring Balance in the Elderly: Preliminary Development of an Instrument," Physiotherapy Canada, vol. 41(6), pp. 304-311, 1989.

[5] P. W. Duncan, D. K. Wiener, J. Chandler, S. Studenski, "Functional Reach: A New Clinical Measure of Balance,” J. Gerontol Med Sci, pp.192-197, 1990.

[6] K. L. Perell, A. Nelson, R. L. Goldman, S. L. Luther, N. Preto-Lewis, and L. Z. Rubenstein, "Fall Risk Assessment Measures: An Analytic Review," Journal of Gerontology, vol. 56A, no. 12, pp. 761-766, 2001.

[7] D. E. Krebs, J. E. Edelstein and S. Fishman, "Reliability of Observational Kinematic Gait Analysis," Physical Therapy, vol. 65, no. 7, pp. 1027-1033, 1985.

[8] J. J. Brunnekreef, C. J. van Uden, S. van Moorsel and J. G. Kooloos, "Reliability of videotaped observational gait analysis in patients with orthopedic impairments," BMC Musculoskeletal Disorders, vol. 16, pp. 17-25, 2005.

[9] M. Alwan and R. A. Felder, "Eldercare Technology for Clinical Practitioners," Humana Press, 2008.

[10] A. Sant'Anna and N. Wicktrom, "Developing a Motion Language: Gait Analysis from Accelerometer Sensor System," pp. 1-8,Pervasive Computing Technology for Healthcare, Pervasive Health 2009.

[11] D. Giansanti, V. Macellari, G. Maccioni, and A. Cappozzo, "Is It Feasible to Reconstruct Body Segment 3-D position and Orientation using Accelerometric Data?" IEEE Transactions on Biomedical Engineering, vol. 50, no. 4, pp. 476-483, 2003.

[12] K. Liu, T. Liu, K. Shibata, Y. Inoue, and R. Zheng, "Novel Approach for Lower Limb Segment Orientation in Gait Analysis Using Triaxial Accelerometers," 
IEEE/ASME International Conference on Advanced Intelligent Mechatronics, AIM, pp. 488 - 492, 2008.

[13] R. W. Selles, M. A. G. Formanoy, J. B. J. Bussmann, P. J. Janssens, and H. J. Starn, "Automated Estimation of Initial and Terminal Contact Timing Using Accelerometers; Development and Validation in Transtibial Amputees and Controls," IEEE Transactions on Neural Systems and Rehabilitation Engineering, vol. 13, no. 1, pp. 81 - 88, 2005.

[14] T. Liu, Y. Inoue, K. Shibata, and X. Tang, "A Wearable Inertial Sensor System for Human Motion Analysis," Proceedings of IEEE International Symposium on Computational Intelligence in Robotics and Automation, CIRA, pp. 409 - 413, 2005.

[ 15 ] J.-A. Lee, S.-H.Cho, J.-W.Lee, K.-H.Lee, and H.-K. Yang, "Wearable Accelerometer System for Measuring the Temporal Parameters of Gait," 29th Annual International Conference of IEEE-EMBS, Engineering in Medicine and Biology Society, EMBC'07, pp. 483 - 486, 2007.

[16] R. K. Ibrahim, E. Ambikairajah, B. G. Celler, and N. H. Lovell, "Time-Frequency Based Features For Classification of Walking Patterns,"15th International Conference onDigitalSignal Processing, pp. 187-190, 2007.

[17] M. Sekine, T. Tamura, T. Fujimoto, and Y. Fukui, "Classification of Walking Pattern Using Acceleration Waveform in Elderly People," Proceedings of the 22nd Annual International Conference of the IEEE in Engineering in Medicine and Biology Society, vol. 2, 2000.

[18] S. J. Morris Bamber, A. Y. Benbasat, D. M. Scarborough, D. E. Krebs, and J. A. Paradiso, "Gait Analysis Using a Shoe-Integrated Wireless Sensor System," IEEE Transactions on Information Technology in Biomedicine, vol. 12, no. 4, 2008.

[19] J. Edmison, M. Jones, T. Lockhart, and T. Martin, "An E-Textile System for Motion Analysis," Stud Health Technology Inform, vol. 108, pp. 292-301, 2004.

[20] M. Pavel, T. L. Hayes, A. Adami, H. Jimison, J. Kaye, "Unobtrusive Assessment of Mobility," 28st Annual International conference of the IEEE EMBs, New York, NY, USA, 2009.

[21] T. L. Hayes, S. Hagler, D. Austin, J. Kaye and M. Pavel, "Unobtrusive Assessment of Walking Speed in the Home Using Inexpensive PIR Sensors," 31st Annual International conference of the IEEE EMBs, pp7248-7251, Minneaplois MN, USA, 2009.

[22] S. Hagler, D. Austin, T. L. Hayes, J. Kaye, M. Pavel, "Unobstrusive and Ubiquitous In-Home Monitoring: A Methodology for Continuous Assessment of Gait Velocity in Elders," IEEE Transactions on Biomedical Engineering, vol. 57, no. 4, 2010. 
[23] M. Alwan, S. Dalal, S. Kell, R. Felder, "Derivation of Basic Human Gait Characteristics From Floor Vibration," Summer Bioengineering Conference, 2003.

[24] K. H. Low, J. W. Tani, T. Chandra, and P. Wang, "Initial Home-Based Foot-Mat Design \& Analysis of Bio-Gait Characteristics to Prevent Fall in Elderly People," Proceedings of the 2009 IEEE International Conference on Robotics and Biomimetics, pp759-764, 2009.

[25] J. K. Aggarwal and Q .Cai, "Human Motion Analysis: A Review," Computer Vision and Image Understanding, vol. 73, pp. 90-102, 1999.

[26] T. B. Moeslund, A. Hilton, V. Kruger, "A Survey of Advances in Vision-Based Human Motion Capture and Analysis," Computer Vision and Image Understanding, vol. 104, pp. 90-126, 2006.

[27] M. S. Nixon, T. N. Tan, and R. Chellappa, "Human Identification Based on Gait," Springer, 2006.

[28] D. Meyer, J. Psl, and H. Niemann, "Gait Classification with HMMs for Trajectories of Body Parts Extracted by Mixture Densities," BMVC, pp. 459-468, 1998.

[29] A. Bissacco, A. Chiuso, Y. Ma, and S. Soatto, "Recognition of human gaits," IEEE Computer Society Conference on Computer Vision and Pattern Recognition (CVPR'01), vol. 2, pp. 52-57, Kauai, Hawaii, USA, December 2001.

[30] C. Bauckage, J. K. Tsotsos, F. E. Bunn, "Detecting Abnormal Gait," Proceedings of The 2nd Canadian Conference on Computer and Robot Vision, pp. 282-288, 2005.

[31] L. Wang, "Abnormal Walking Gait Analysis Using Silhouette-Masked Flow Histograms," vol. 3, pp473-476, 18th International Conference on Pattern Recognition, ICPR 2006.

[32] H. NAIT-CHARIF and S. McKenna, "Activity Summarisation and Fall Detection in a Supportive Home Environment," Intl. Conf. on Pattern Recognition , 2004, Pattern Analysis and Applications Journal. Published online: 20 May 2005, http://nccastaff.bournemouth.ac.uk/hncharif/Publications/summarizingcontextual.pd $\underline{\mathrm{f}}$

[33] J. GAO, H. WACTLAR, A. HAUPTMANN, "Combining Motion Segmentation with Tracking for Activity Analysis," Intl. Conf. Automatic Face and Gesture Recognition, pp. 699-704, 2004.

[34] B. P. L. Lo, J. L. Wang and G-Z Yang, "From Imaging Networks to Behavior Profiling: Uniquitous Sensing for Managed Homecare of the Elderly," Adjunct Proceedings of the 3rd International Conference on Pervasive Computing (PERVASIVE 2005), pp. 101-104, 2005. 
[35] M. Goffredo, R. D. Seely, J. N. Carter and M. S. Nixon, "Markerless View Independent Gait Analysis with Self-camera Calibration," , 8th IEEE International Conference on Automatic Face \& Gesture Recognition, pp. 1-6, 2008.

[36] J. Saboune and F. Charpillet, "Markerless Human Motion Capture for Gait Analysis," ArXiv Computer Science e-prints, cs/0510063, October, 2005.

[37] M. Goffredo, M. Schmid, S. Conforto, and T. D'Alessio, "A Markerless Sub-pixel Motion Estimation Technique to Reconstruct Kinematics and Estimate the Center of Mass in Posturography," Medical Engineering \& Physics, vol. 28, pp. 719-726, 2006.

[38] S. J. Allin, C. Beach, A. Mitz and A. Mihailidis, "Video Based Analysis of Standing Balance in a Community Center," Proc. 30th Annual International Conference of the IEEE Engineering in Medicine and Biology Society, Vancouver, British Columbia, Canada, pp. 4531-4534, 2008.

[39] S. Allin, and A. Mihailidis, "Sit to Stand Detection and Analysis," $A A A I$ in Eldercare: New Solutions to Old Problems, Washington DC, November 7-9, 2008.

[40] M. Goffredo, M. Schmid, S. Conforto, M. Carli, A. Neri, and T D'Alessio, "Markerless Human Motion Analysis in Gauss-Laguerre Transform Domain: An Application to Sit-To-Stand in Young and Elderly People," vol. 13, no. 2, IEEE Transactions on Information Technology in Biomedicine, 2009.

[41] J. Pansiot, D. Stoyanov, D. Mcllwraith, B. P. L. Lo, and G. Z. Yang, "Ambient and Wearable Sensor Fusion for Activity Recognition in Healthcare Monitoring System," In IFMBE proceedings of the 4th International Workshop on Wearable and Implantable Body Sensor Networks 2007 (BSN), pp. 208-212, Aachen, Germany, 2007.

[42] A. M. Tabar, A. Keshavarz, H. Aghajan, "Smart Home Care Network using Sensor Fusion and Distributed Vision-Based Reasoning," ACM Multimedia Workshop on Video Surveillance and Sensor Networks (VSSN), 2006.

[43] P. Siciliano, A. Leone, G. Diraco, C. Distante, M. Malfatti, L. Gonzo, M. Grassi, A. Lombardi, G. Rescio, and P. Malcovati, "A Networked Multisensor System for Ambient Assisted Living Application," 3rd International Workshop on Advances in Sensor and Interface, 2009.

[44 ] M. Skubic, G. Alexander, M. Popescu, M. Rantz, \& J. Keller, "A smart home application to eldercare: current status and lessons learned," Technology and Health Care 17(3), pp. 183-201, 2009 
[45] M. Rantz, K. Marek, M. Aud, R. Johnson, D. Otto, R. Porter, "TigerPlace: A New Future for Older Adults", Journal of Nursing Care Quality, vol. 20, no. 1, pp. 1-4, 2005.

[46] C. Stauffer and W. Grimson, "Adaptive Background Mixture Models for Real-Time Tracking," in Computer Society Conference on Computer Vision and Pattern Recognition, 1999.

[47] N.M. Oliver, B. Rosario, and A.P. Pentland, "A Bayesian Computer Vision System for Modeling Human Interactions," IEEE Transactions on Pattern Analysis and Machine Intelligence, vol. 22, no. 8, pp. 831-843, 2000.

[48] L. Li, W. Huang, I. Gu, and Q. Tian, "Foreground Object Detection from Videos Containing Complex Background," in Proceedings of the eleventh ACM international conference on Multimedia, Berkeley, CA, USA, pp. 2-10, 2003.

[49] R. H. Luke, "Moving Object Segmentation from Video Using Fused Color and Texture Features in Indoor Environments," Technical Report, CIRL, University of Missouri, 2008.

[50] D. Anderson, R. H. Luke, J. M. Keller, M. Skubic, M. Rantz and M. Aud, "Linguistic Summarization of Video for Fall Detection Using Voxel Person and Fuzzy Logic," Computer Vision and Image Understanding, vol. 113, pp. 80-89, 2008.

[51] Jean-Yves Bouguet, "Camera Calibration Toolbox for Matlab," http://www.vision.caltech.edu/bouguetj/calib doc/.

[52] X. Wang, T. X. Han and S. Yan, "An HOG-LBP Human Detector with Partial Occlusion Handling," IEEE International Conference on Computer Vision (ICCV 2009), Kyoto, 2009.

[53] K. E. Webster, J. E. Wittwer, Julian F. Feller, "Validity of GAITRite Walkway System for the Measurement of Averaged and Individual Step Parameters of Gait," Gait and Posture, vol. 22, pp. 317-321, 2005.

[54] F. Wang, E. Stone, W. Dai, M. Skubic, and J. Keller, "Gait Analysis and Validation Using Voxel Data," Proceedings, 31st Annual International Conference of the IEEE Engineering in Medicine and Biology Society, pp. 6127-6130, 2009.

[55] “Gait Disorders in the Elderly,” Merck Manual Professional, 2010.

[56] G. Yogev, M. Plotnik, C. Peretz, N. Giladi, J. Hausdorff, "Gait Asymmetry in Patients with Parkinson's Disease and Elderly Fallers: When Does the Bilater 
Coordination of Gait Require Attention?" Exp Brain Res, vol. 177, pp. 336-346,, 2007.

[57] D. Anderson, R. H. Luke, E. Stone, and J. M. Keller, "Fuzzy Voxel Model for Human Activity Analysis," International Fuzzy Systems Association (IFSA), Lisbon, Portugal, July 2009.

[58] K. Traldsen, T. Askim, O. Sletvold, E. K. Einarsen, K. G. Bjastad, B. Indredavik, J. L. Helbostad, "Evaluation of a Body-Worn Sensor System to Measure Physical Activity in Older People With Impaired Function," Physical Therapy, vol. 91, no. 2, pp. 277-285, 2011.

[59] J. M. Simpson, C. Worsfold, E. Reilly and N. Nve, "A Standard Procedure for Using TURN180: testing dynamic postural stability among elderly people," Physiotherapy, vol. 88 (6), pp. 342-53, 2002.

[60] H. M. Parson, “What Happened at Hawthorne?” Science, 193, pp. 922-932, 1974.

[61] D. De Amici, C. Klersy, F. Ramajoli, L. Brustua, and P. Politi "Impact of the Hawthorne Effect in a Longitudinal Clinical Study: The Case of Anesthesia," Controlled Clinical Trials, vol.21, no.2, pp.103-14, 2000.

[62] J. Yu, "The Significance of Walking Speed in Physical Function Among a Group of Community Dwelling Older Adults," Ph.D thesis, University of MissouriColumbia. 2008.

[63] Y. Barak, R. C. Wagenaar, K. G. Holt, "Gait Characteristics of Elderly People with a History of Falls: A Dynamic Approach," Physical Therapy, vol. 86, no. 11, pp. 1501-1510, 2006.

[64] G. Demiris, D. P. Oliver, J. Giger, M. Skubic, and M. Rantz, "Older Adults' Privacy Considerations for Vision Based Recognition Methods of Eldercare Applications," Technology and Health Care, vol. 17, no. 1, pp. 41-48, 2009

[65] E. E. Stone, and M. Skubic , "Silhouette classification using pixel and voxel features for improved elder monitoring in dynamic environments," Proceedings, 2011 IEEE International Conference on Pervasive Computing and Communications Workshops (PERCOM Workshops), pp. 655-661, 2011. 


\begin{abstract}
VITA
Fang Wang received the B.S.E.E. degree from Nankai University, Tianjin, China, in 1997, and the M.S.E.E degree from Southern Methodist University, Dallas, TX in 2000, with a thesis in low frequency noise in submicrometer MOSFETs. From 2001-2006, she worked as Sr. Device Modeling and Characterization Engineer at Motorola Inc, and later Freescale Semicondutor Inc. She received her Ph.D in Electrical and Computer Engineering, University of Missouri in 2011. Her research interests include eldercare technology, computational Intelligence, computer vision, and image processing.
\end{abstract}

